\title{
Acute liver failure is regulated by MYC- and microbiome-dependent programs
}

\author{
Aleksandra A. Kolodziejczyk', Sara Federici ${ }^{1}{ }^{1}$, Niv Zmora ${ }^{1,2,3}$, Gayatree Mohapatra1, \\ Mally Dori-Bachash', Shanni Hornstein', Avner Leshem ${ }^{1,4}$, Debby Reuveni' ${ }^{2,3}$, Ehud Zigmond ${ }^{2,3}$, \\ Ana Tobar ${ }^{2,5}$, Tomer Meir Salame ${ }^{6}$, Alon Harmelin7, Amir Shlomai ${ }^{2,8}$, Hagit Shapiro ${ }^{1}$, Ido Amit $\mathbb{C}^{1 凶}$ \\ and Eran Elinav $(\mathbb{1})^{1,9} \bowtie$
}

Acute liver failure (ALF) is a fulminant complication of multiple etiologies, characterized by rapid hepatic destruction, multi-organ failure and mortality. ALF treatment is mainly limited to supportive care and liver transplantation. Here we utilize the acetaminophen (APAP) and thioacetamide (TAA) ALF models in characterizing 56,527 single-cell transcriptomes to define the mouse ALF cellular atlas. We demonstrate that unique, previously uncharacterized stellate cell, endothelial cell, Kupffer cell, monocyte and neutrophil subsets, and their intricate intercellular crosstalk, drive ALF. We unravel a common MYC-dependent transcriptional program orchestrating stellate, endothelial and Kupffer cell activation during ALF, which is regulated by the gut microbiome through Toll-like receptor (TLR) signaling. Pharmacological inhibition of MYC, upstream TLR signaling checkpoints or microbiome depletion suppress this cell-specific, MYC-dependent program, thereby attenuating ALF. In humans, we demonstrate upregulated hepatic MYC expression in ALF transplant recipients compared to healthy donors. Collectively we demonstrate that detailed cellular/genetic decoding may enable pathway-specific ALF therapeutic intervention.

A LF constitutes a medical emergency, in which a fulminant functional hepatic insufficiency leads to a rapid clinical deterioration and a high mortality rate in the absence of liver transplantation ${ }^{1}$. Multiple infectious, immune, metabolic and neoplastic diseases may manifest as ALF. Of these, APAP overdose is a leading cause for ALF in developed countries ${ }^{2,3}$. A comprehensive, high-resolution cellular characterization of the events leading to liver insufficiency remains unexplored, resulting in a lack of sufficient global understanding of the molecular basis of ALF and identification of ALF therapeutic targets. Consequently, ALF treatment remains limited and mostly supportive. Equally elusive is the contribution of the gut microbiome to ALF. While this ecosystem has been repeatedly shown to influence hepatic physiology, drug metabolism (including APAP) ${ }^{4}$ and disease processes, including hepatocellular carcinoma ${ }^{5}$, primary sclerosing cholangitis ${ }^{6}$, nonalcoholic fatty liver disease ${ }^{7}$ and alcoholic liver disease ${ }^{8}$, little is known about the potential cellular and molecular modulatory functions of the gut microbiome in ALF.

\section{Results}

A single-cell transcriptomic atlas of liver in healthy and ALF settings. We began our cell-specific exploration of the liver in homeostasis and ALF by characterizing healthy and liver-damage adult (8-week-old) specific pathogen-free (SPF)-housed C57BL6 mice, using the acute APAP and TAA ALF models (Methods, Fig. 1a and Extended Data Fig. 1a). Of note, both TAA and APAP elicit oxidative stress through similar mechanisms ${ }^{2,9}$, and an ensuing intense liver inflammation, further contributing to liver damage ${ }^{10}$.
To profile the hepatic nonparenchymal cellular populations in naïve and ALF settings, we depleted hepatocytes from liver cellular samples by centrifugation. Half of the resultant cellular fraction was further enriched for hepatic stellate cells (HSCs) by flow cytometry gating on intrinsic retinoid fluorescence of this cell population (Extended Data Fig. 1b) ${ }^{11}$. The remaining half remained unaffected, to enable unbiased quantification using single-cell genomics. Using droplet-based, single-cell RNA sequencing (RNA-seq), we analyzed 6,592 cells from three 8-week-old healthy male C57BL6 mice housed under SPF conditions at our facility, as well as 10,609 cells from APAP-treated mice and 8,500 from TAA-treated mice (Fig. 1b). Using hierarchical clustering, we identified 40 different cell populations that could be divided into six major types: immune cells, endothelial cells, HSCs, hepatocytes, cholangiocytes and mesothelial cells, collectively resulting in a high-resolution liver cell atlas (Methods and Supplementary Figs. 1-12). We annotated cell clusters using conventional markers, major histocompatibility complex II (MHCII) expression and by comparison of their specific gene expression patterns to the Immgen database ${ }^{12}$ (Fig. 1c and Extended Data Fig. 1c).

Within stellate cells we found four distinct populations and, based on their markers, we classified these as Lrat ${ }^{\text {high }}$ quiescent stellate cells, Col1a1-positive fibrotic stellate cells, Acta2-positive ALF-activated stellate cells (referred to as AAs) and cycling stellate cells. In the endothelial cell population, we identified three clusters bearing different transcriptional signatures depending on their localization - the most abundant being a liver sinusoidal endothelial cell (LSEC) population and two additional, smaller

\footnotetext{
'Immunology Department, Weizmann Institute of Science, Rehovot, Israel. ${ }^{2}$ The Sackler Faculty of Medicine, Tel Aviv University, Tel Aviv, Israel. ${ }^{3}$ The Research Center for Digestive Tract and Liver Diseases, Tel Aviv Sourasky Medical Center, Tel Aviv, Israel. ${ }^{4}$ Department of Surgery, Tel Aviv Sourasky Medical Center, Tel Aviv, Israel. ${ }^{5}$ Department of Pathology, Rabin Medical Center, Beilinson Hospital, Petah Tikva, Israel. ${ }^{6}$ Department of Biological Services, Weizmann Institute of Science, Rehovot, Israel. 'Department of Veterinary Resources, Weizmann Institute of Science, Rehovot, Israel. ${ }^{8}$ Department of Medicine D and the Liver Institute, Rabin Medical Center, Beilinson Hospital, Petah Tikva, Israel. ' $D i v i s i o n$ of Cancer-Microbiome Research, DKFZ, Heidelberg, Germany. 凶e-mail: ido.amit@weizmann.ac.il; eran.elinav@weizmann.ac.il
} 
a

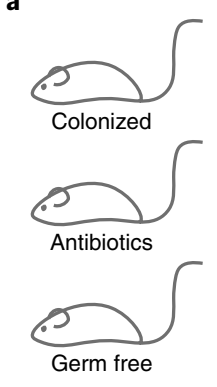

Microbiome state

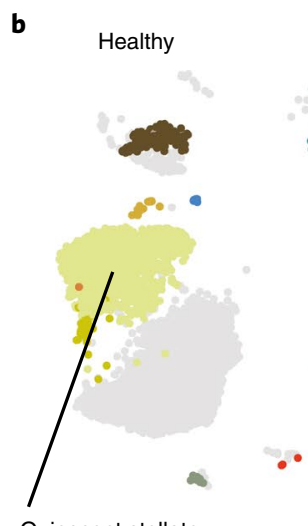

Quiescent stellate

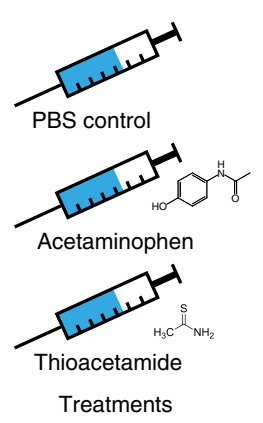

Treatments
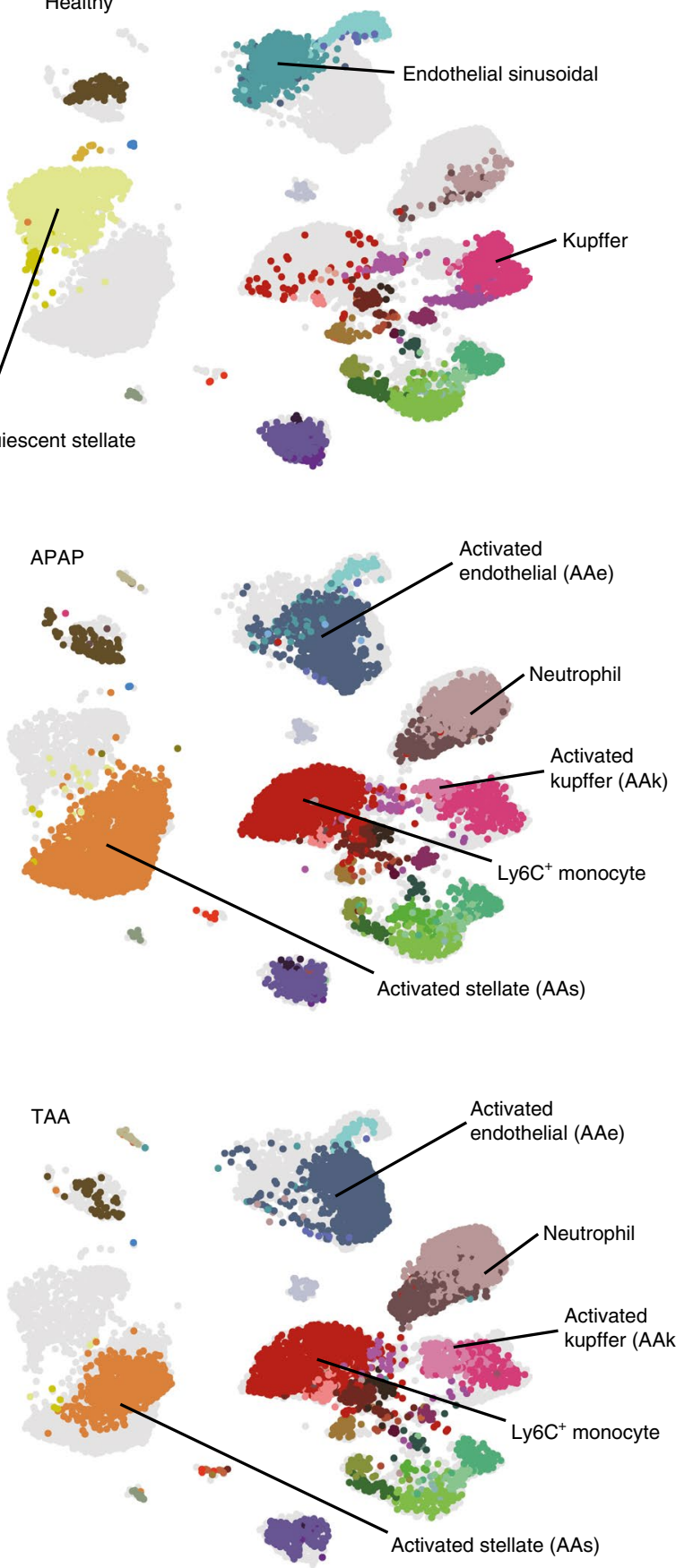

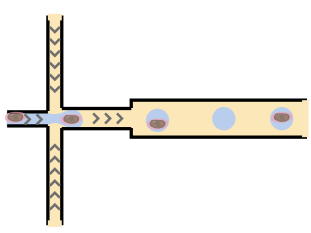

Single-cell RNA-seq

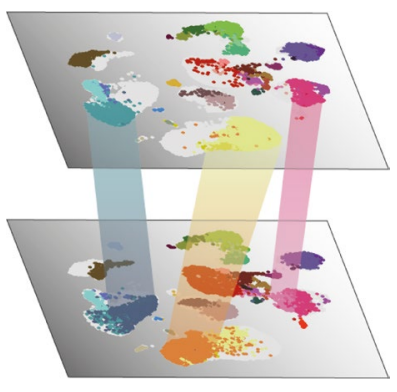

Population shift analysis

c

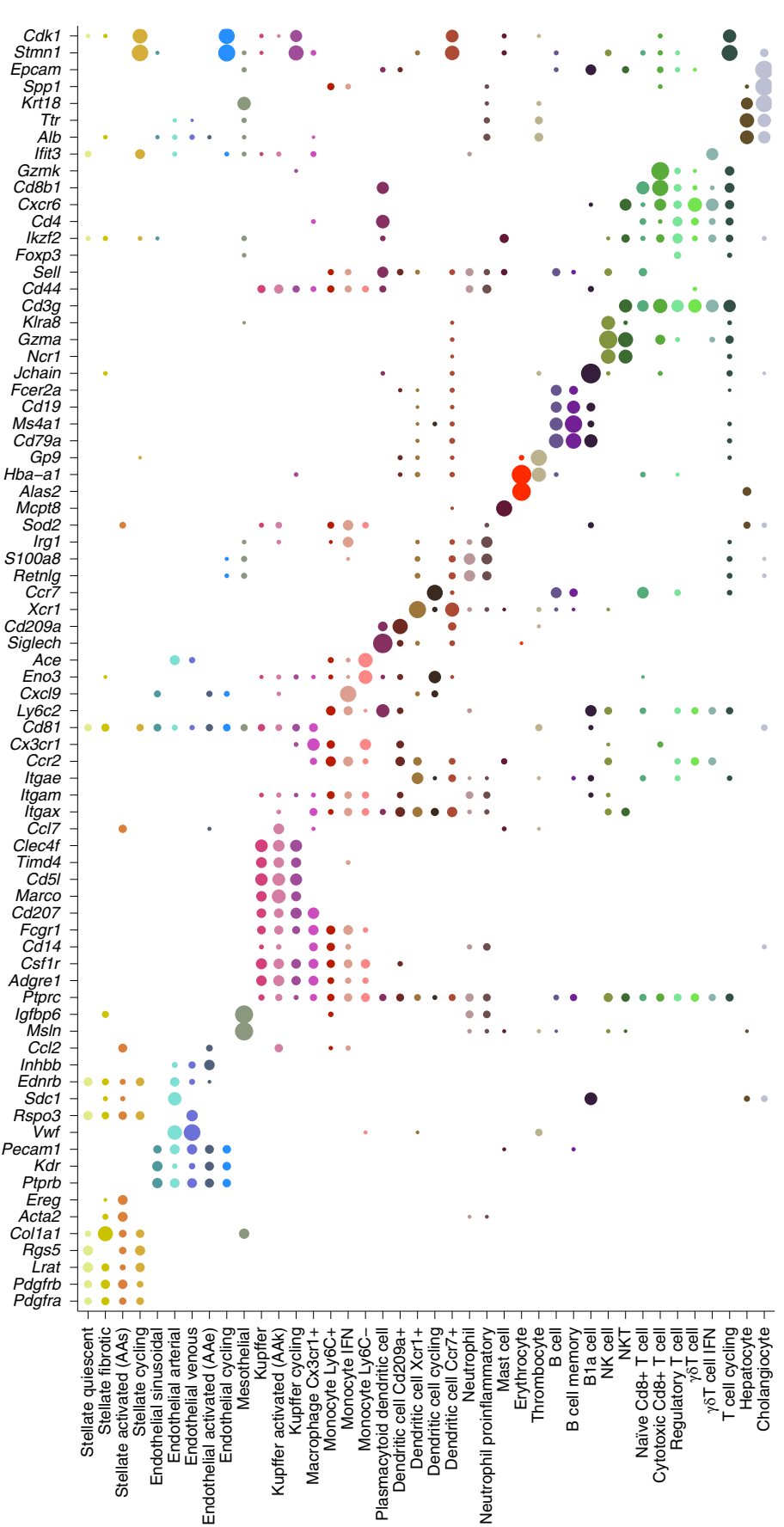

Fig. 1 | Mouse liver cell census in acute liver failure mouse models. a, Experiment schematic. b, Uniform manifold approximation and projection visualization of cell clusters in healthy $(n=3)$, APAP-treated $(n=4)$ and TAA-treated specific pathogen-free (SPF) mice $(n=4)$. To aid in comparison, the gray background shows all cells. c, Key markers used to describe cluster identity and link it to cell type. 
populations originating from veins and arteries ${ }^{13,14}$. Furthermore, we found a population of ALF-activated endothelial cells (referred to as AAe), described below in detail. The expression of Timd4 and Clec $4 f^{15}$ was used as a marker of Kupffer cells within which we identified two populations, one quiescent and one ALF-activated (referred to as AAk).

Response to ALF-inducing toxic insult, in both models, was characterized by initial changes in the three newly identified resident liver cells (AAs, AAe and AAk; Fig. 1b), which coincided with a similar decrease in abundance of the respective nonactivated cell states, suggesting that these cells are activated counterparts rather than recruited populations. Additionally, infiltrating immune cells were also observed ${ }^{16}$, including infiltrating Ly6C-positive monocyte populations noted in both the TAA and APAP models and infiltrating neutrophil populations noted to be more pronounced in the TAA model.

Response of resident populations to ALF. Upon induction of ALF, the activated state in the three hepatic resident cell subsets (AAs, AAe and AAk) corresponded to a distinct gene expression profile compared with their quiescent transcriptomic profile.

AAs. Previously, HSC states were characterized mainly in health and in chronic disease models such as nonalcoholic fatty liver disease and fibrosis, as reported both in bulk and also, to a limited extent, at the single-cell level ${ }^{17-19}$. Importantly, Colla1 and Acta2 are considered hallmark markers of HSC in disease, often called 'myofibroblasts ${ }^{17,20}$, suggestive of two features: a contractile ('myo') phenotype mediated by expression of stress fiber genes, and an extracellular, matrix-secreting ('fibroblast') phenotype, especially of collagens ${ }^{21}$.

Interestingly we found that, in both ALF models, expression of Colla1 and Acta2 was mutually exclusive and that only Acta2-positive AAs cells, but not Colla1-positive fibrotic cells, were strongly upregulated upon ALF-induced activation (Fig. 2a). In both the APAP and TAA model, stellate cells assumed an Acta2-positive AAs state while the population of Col1a1-positive fibrotic stellate cells was downregulated (Fig. 2b). The fibrotic stellate cell population is characterized by the highest expression of collagens in comparison to other HSC subtypes (Fig. 2c). Similarly, several genes from the Gene Ontology (GO) term extracellular matrix (GO:0031012) were also upregulated in AAs cells in comparison to quiescent cells (Fig. 2d), while genes belonging to the GO term 'stress fiber' were upregulated in ALF-activated stellate cells, including the hallmark gene Acta2 (Fig. 2e). Collectively, these results suggest that, in addition to a quiescent stellate cell state, there are at least two distinct phenotypes that stellate cells can assume, namely a Colla1-expressing fibroblast state and an immunomodulatory, contractile Acta2-positive state. In the ALF models tested in our studies, these transcriptional programs did not coexist within a single cell.

To further dissect the function of AAs cells, we performed differential expression analysis between quiescent and activated stellate cell populations and found that 421 genes induced by the toxic ALF insult are related to gene expression and translation (Fig. 2f), suggestive of a potentially increased protein production in these cells. This observation coincided with the higher number of detected transcripts in the activated population (Extended Data Fig. 1d-e), including secreted factors such as Ccl2, Ccl7, Csf1, Tagln, Tagln2 and Thbs1 (Fig. 2g). Within the cytokines induced in AAs during ALF were members of the interleukin-6 family, including Il6, Il11 and Lif $^{22,23}$. Interestingly, receptors for these interleukins were expressed by different cell types, suggesting that responsiveness to interleukin-6 family cytokines may represent a possible 'division of labor' in cellular signaling (Fig. $2 \mathrm{~h}$ ).

Gene Ontology enrichment analysis of AAs also revealed terms associated with cell death: among upregulated genes within these terms we found Trp53 and Cdkn1a. In the presence of stress, p53 induces expression of the gene Cdkn1a that triggers cell cycle arrest, leading to senescence or apoptosis ${ }^{24}$. Increased cellular transcriptional activity, coupled with markedly induced senescence-associated secretory phenotype (chemokines, Timp1 and Ereg) expression collectively suggested that ALF-associated AAs cells may feature senescence rather than apoptosis ${ }^{25}$. Interestingly, a trend towards a lower number of proliferating cells was noted in ALF in all resident populations, further supporting the notion that liver cells may undergo cell cycle arrest (Extended Data Fig. 2a).

Importantly, AAs cells in ALF induced by either APAP or TAA clustered together, suggesting that differences in stellate cell activation states between these conditions are somewhat minor. To examine potential molecular differences between the two models, we performed differential expression with DESeq2 using pseudobulk from single-cell populations in the APAP and TAA samples (Extended Data Fig. 2b), highlighting 45 genes overlapping with the stellate cell activation signature-for example, Il11, Itga5 and Timp1 were higher in APAP while genes related to stress response, such as Mt1 or Hifla, were higher in TAA. Together, this suggests that key transcriptional changes involving cytokines and extracellular matrix proteins are similarly upregulated in ALF regardless of the liver insult.

AAe cells. Endothelial cells regulate blood flow in the liver through vasoconstriction, form a barrier for molecules and immune cell liver trafficking through regulation of fenestration and partake in blood clearance through endocytosis ${ }^{26}$. Liver sinusoidal endothelial cells (Fig. 2i), but not venous and arterial endothelial cells (Extended Data Fig. 2a), assumed an activated phenotype upon

Fig. 2 | Activation of resident cell population in ALF. a, Violin plots showing normalized and scaled expression of Col1a1 and Acta2 in stellate cells from three clusters: quiescent, fibrotic and AAs. b. Percentage of stellate cell populations in all stellate cells in control $(C T R L)(n=3)$, APAP-treated $(n=4)$ and TAA-treated ( $n=4$ ) mice; significance was determined using a two-sided Wilcoxon test. Boxplot shows 25 th to 75 th percentiles with the 50 th denoted by a line; whiskers show $1.5 \times$ interquartile range, or maximum or minimum if smaller. c, Balloon plots showing mean normalized and scaled expression of collagens in stellate cell subpopulations. d, Heatmap showing z-score for expression of genes from extracellular matrix GO category GO:0031012 that are significantly upregulated in AAs. e, Heatmap showing z-score for expression of genes from GO category stress fiber GO:0001725 that are significantly upregulated in AAs. f, GO term enrichment analysis of genes upregulated in AAs in comparison to quiescent cells. GO analysis was performed with GProfiler using standard settings; Pvalues shown are corrected for multiple hypothesis testing using the g:SCS algorithm. $\mathbf{g}$, Violin plots showing expression of chemokines, cytokine and extracellular matrix regulators in stellate cell populations. $\mathbf{h}$, Balloon plot showing normalized and scaled expression of IL6 family cytokines and their receptors in all cell types. $\mathbf{i}$, Percentage of endothelial cell populations in all endothelial cells in control ( $n=3$ ), APAP-treated $(n=4)$ and TAA-treated $(n=4)$ mice; significance was determined using a two-sided Wilcoxon test. Boxplot defined as in $\mathbf{b}$. $\mathbf{j}$, GO term enrichment analysis of genes upregulated in AAe in comparison to sinusoidal endothelial cells. GO analysis was performed as in $\mathbf{f}$. $\mathbf{k}$, Percentage of Kupffer cell populations in immune cells in control $(n=3)$, APAP-treated $(n=4)$ and TAA-treated $(n=4)$ mice; significance was determined using a two-sided Wilcoxon test. Data points as in b; boxplot defined as in b. I, GO term enrichment analysis of genes upregulated in AAk in comparison to Kupffer cells. GO analysis was performed as in $\mathbf{f}$. $\mathbf{m}$, Balloon plots showing significantly upregulated ligands in populations of stellate, endothelial and Kupffer cells and corresponding receptors, and their normalized and scaled expression in all cell types. 
ALF induction. Gene Ontology term enrichment analysis of upregulated genes in AAe revealed terms related to gene expression and terms associated with vascular remodeling (Fig. 2j). Upregulated transcripts suggested that endothelial cells are active participants in the immune response $(C c l 2, T g f b 1$ and $I n h b b)$, extracellular matrix remodeling (Adamts1, Tgm2, Col4a1 and Col4a1) and regenerative

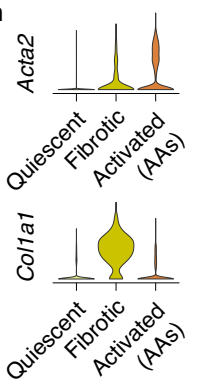

d

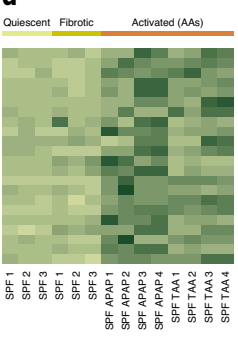

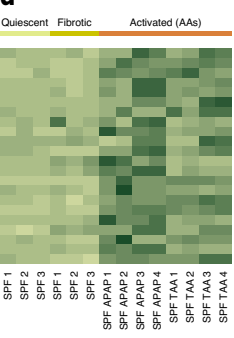

b

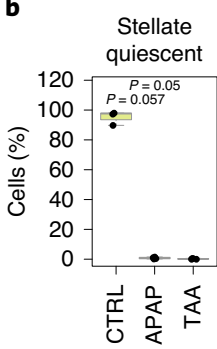

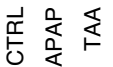

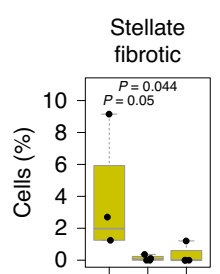

品竞 c

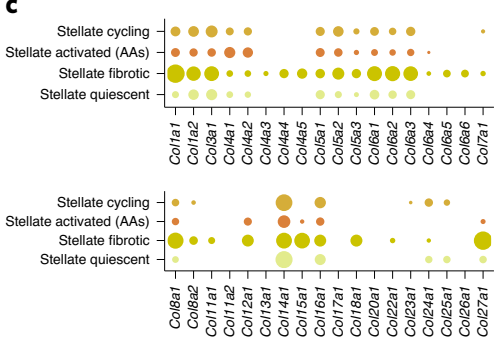

g
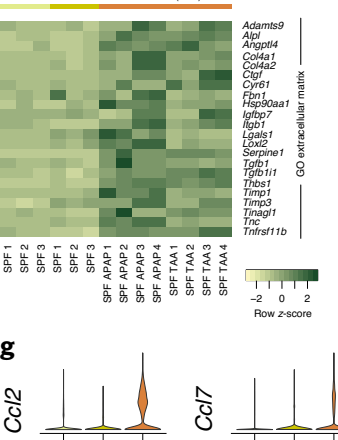

e

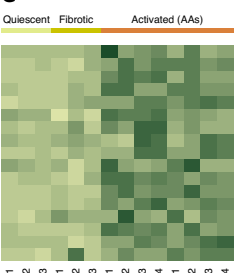

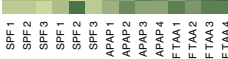

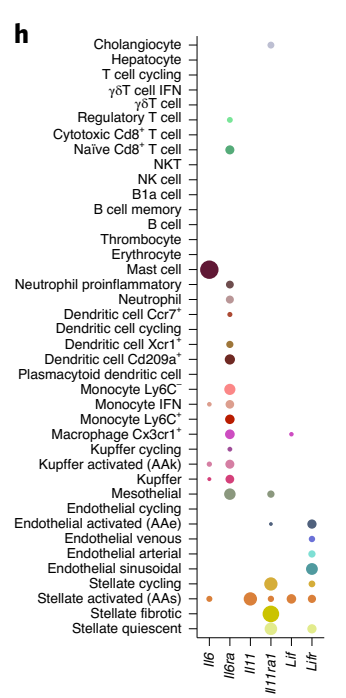

i
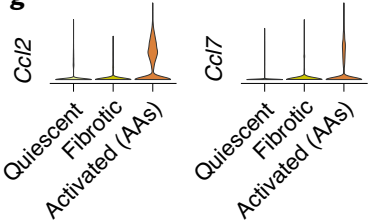

$5+1$
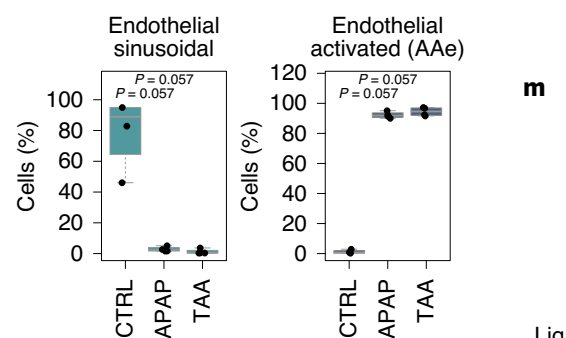

j

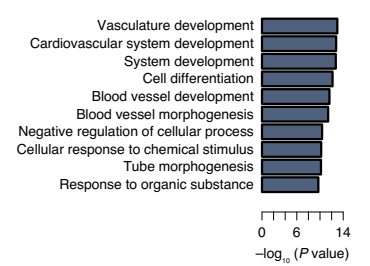

k

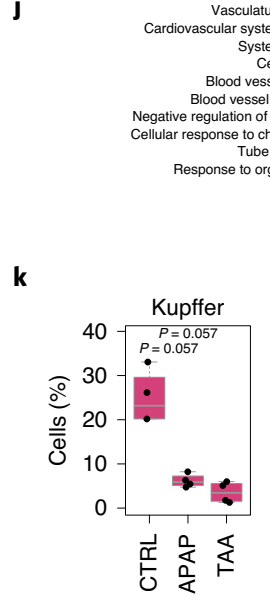

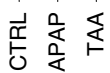

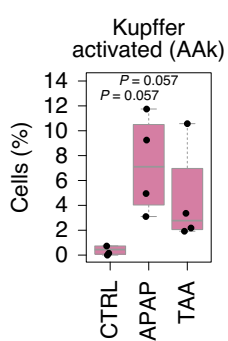

I

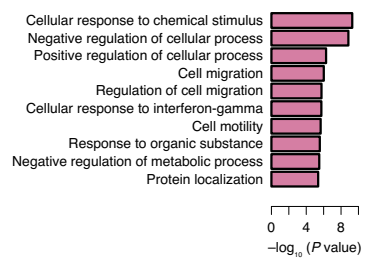

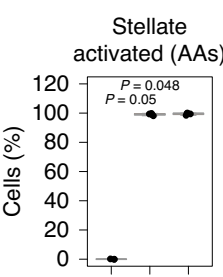

品是 f

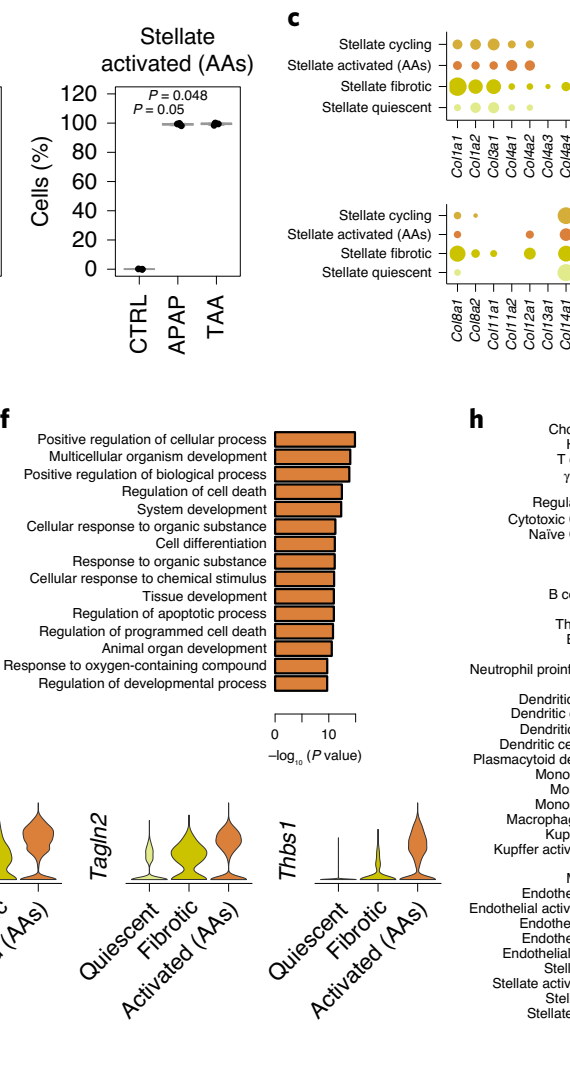

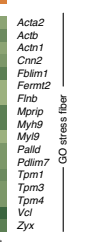
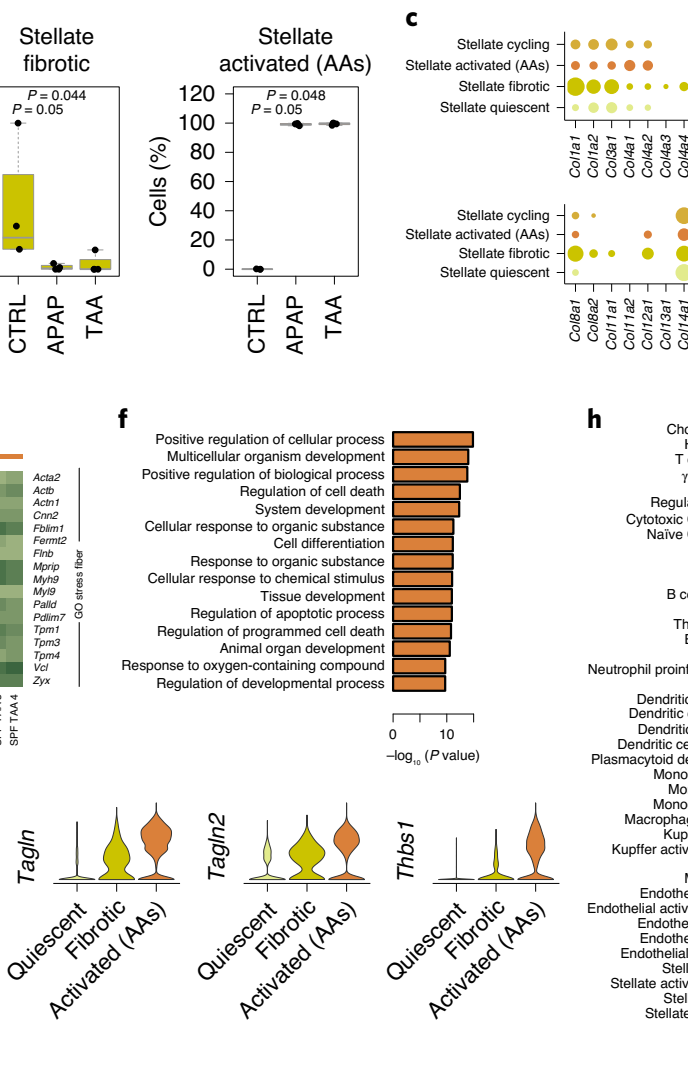

Receptors
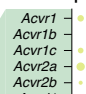

Acvir
Acvi
Ban
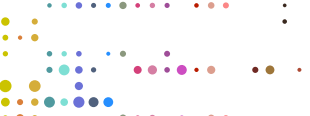

$\because \ldots$

Ligands

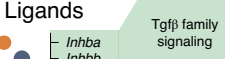

signaling

.

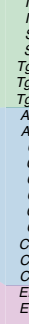


processes (Tek and Wnt2) (Extended Data Fig. 2c). Comparison of AAe between the APAP and TAA models revealed 101 differentially expressed genes, including Fos and Junb, that function in stress response $^{27}$ (Extended data Fig. 2d). This suggests that TAA may elicit more oxidative stress than APAP.

AAk cells. Acute liver failure was associated with activation of, on average, 51.5\% of Kupffer cells (Fig. 2k). Gene Ontology analysis of upregulated genes revealed terms related to chemotaxis, cell migration, immune response and apoptosis (Fig. 21 and Extended Data Fig. 2e). Similar to stellate cells, apoptosis-related terms are probably associated with cell cycle arrest because we did not observe hallmarks of apoptosis such as increase in the percentage of mitochondrially encoded transcripts (Extended Data Fig. 3a). Kupffer cell activation was similar in APAP- and TAA-induced ALF, with 26 genes differentially expressed between the two disease models, including several interferon-responsive genes.

ALF-associated cell-to-cell signaling. Hepatic communication networks involving co-residing cells are frequently altered in liver disease ${ }^{28,29}$. To explore certain liver cell-to-cell communication motifs in steady state and ALF, we filtered differentially expressed genes between AAs, AAe and AAk and their quiescent equivalents, for ligands from a dataset curated by Ramilowski et al. ${ }^{30}$. We identified 18 ligands that can be grouped into the categories TGF $\beta$ ligands, chemokines, cytokines and growth factors, then identified matching receptors for these ligands. Due to redundancy in ligand-receptor interactions (for example, $\mathrm{Ccl} 2$ chemokine was shown to bind to $C c r 1, C c r 2, C c r 3$ and $C c r 4$ ), we grouped receptors into the same functional categories as ligands and found major signaling modules: chemokines target mostly the immune compartment, TGF $\beta$ targets mainly stellate and endothelial cells while growth factors and cytokines seem to potentially affect all cell types (Fig. $2 \mathrm{~m}$ ). The roles and contributions of these cell- and ligand-specific 'division of labor' networks in steady state and liver disease merit further studies.

Liver failure-associated cellular infiltration. We next investigated the characteristics of infiltrating cells during ALF. Indeed, in parallel to reduced cell proliferation of resident cells during ALF (Extended Data Fig. 2a), we identified populations of expanded hepatic subsets that did not have corresponding quiescent counterparts and thus probably represented infiltrating cells. Ly6C-positive monocytes expressed $\mathrm{C} c \mathrm{r} 2$; its ligand, $\mathrm{C} c \mathrm{l} 2$, was previously reported to be responsible for monocyte recruitment ${ }^{31}$ (Extended Data Fig. 3b). In contrast, the neutrophil infiltrating fraction did not express Ccr2 and was probably recruited via a different mechanism ${ }^{32,33}$, possibly the highly expressed Ccr1 or Cxcr2 (Fig. 2m and Extended Data Fig. $3 \mathrm{c}-\mathrm{d}$ ). Because both the infiltrating ALF-associated neutrophil and monocyte subsets were heterogeneous, we further dissected them in decoding potential distinct functional roles of their subsets.
Heterogeneity of neutrophils. We identified two neutrophil subpopulations (Fig. 3a), the larger subset representing classical, tissue-resident neutrophils ${ }^{34}$ and the smaller expressing $\mathrm{Ccl} 3, \mathrm{Ccl} 4$, Cxcl 2 and Csf1, suggesting that these cells were probably the proinflammatory subtype ${ }^{35}$. These neutrophils also expressed $\mathrm{Nfe} 2 \mathrm{l} 2$ encoding the NRF2 transcription factor, known for regulation of the antioxidant transcriptional program ${ }^{36}$. Interestingly, Cxcr2 was downregulated upon neutrophil activation, possibly suggesting that it may be involved in mediation of infiltration (Fig. 3b). Neutrophil infiltration and activation were more pronounced in TAA-treated as compared to APAP-treated mice (Fig. 3c).

Heterogeneity of Ly6C-positive monocytes. Ly6C-positive monocytes have been suggested to infiltrate the liver in a number of pathologies $^{37}$. We identified two populations of Ly6C-positive monocytes: the main population of 3,507 cells massively infiltrated the liver in ALF, while a small subpopulation of only 71 cells was not affected by the disease. Differential expression, GO and transcription factor binding site analyses revealed that the small population is most similar to Ly6C-positive monocytes and that it features an upregulated response to interferon (Fig. $3 \mathrm{~d}-\mathrm{i}$ ). The main population of Ly6C-positive monocytes exhibited further underlying heterogeneity. Diffusion maps ${ }^{38}$ revealed that gene expression heterogeneity in this monocyte cluster stems from two processes, one consisting of monocyte homing to the liver and the other induction of MHCII complex gene expression (Fig. 3j). Of note, monocyte homing led to a gradual loss of expression of $L y 6 c 2$ and Sell, coupled with increased expression of Cxcl16, C1qa, Hmox1 and cathepsins (Fig. 3k).

Molecular activation patterns in ALF-induced resident cellular subsets. Importantly, some of the upregulated genes in AAs, AAe and AAk, such as Ccl2, Nfe2l2 or Mt1, were common to these three cell types, suggesting a possible common activation signature in ALF. Indeed, we identified as many as 77 commonly expressed genes in AAs, AAe and AAk (Fig. 4a). A Monte Carlo simulation estimating the odds of such a commonality being random, using $10^{9}$ iterations, did not observe a single instance of overlap with as many genes, strongly hinting at a common transcriptional response program underlying this expression pattern. An enrichment analysis of transcription factor binding site motifs within the promoters of this gene set yielded multiple different MYC binding motifs, suggesting that it may be a regulator of this response (Fig. 4b). To further corroborate this enrichment, we performed permutation analysis of a number of MYC binding sites within 77 randomly chosen genes: $10^{10}$ iterations resulted in the distribution of a mean 197 binding sites and a maximum value of 343 binding sites, while within the common activation signature we discovered $402 \mathrm{MYC}$ binding sites (Extended Data Fig. 4a). Furthermore, expression of the Myc transcript also trended towards upregulation in ALF but did not reach statistical significance (Extended Data Fig. 4b). At the protein level,

Fig. 3 | Heterogeneity of infiltrating cells in ALF. a, $t$-distributed stochastic neighbor embedding ( $t$-SNE) depicting two populations of neutrophils and an example of genes specific for the subpopulations. b, Violin plots showing normalized and scaled expression levels of chemokines, cytokines and oxidative stress-response genes in neutrophil cell populations. FDR corrected $P<10^{-32}$. c, Percentage of neutrophil populations in immune cells in control ( $\left.C T R L\right)$ $(n=3)$, APAP-treated $(n=4)$ and TAA-treated $(n=4)$ mice; significance was determined using a two-sided Wilcoxon test. Boxplots defined as in Fig. 2 b. d, $t$-SNE depicting two populations of Ly6C-positive monocytes and an example of genes specific for the subpopulations. e, Barplot showing number of

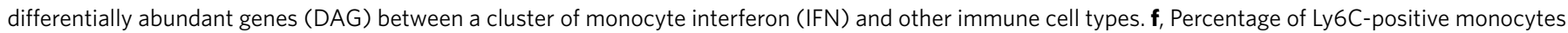
in immune cells in control $(n=3)$, APAP-treated $(n=4)$ and TAA-treated $(n=4)$ mice; significance was determined using a two-sided Wilcoxon test. Boxplots defined as in Fig. 2 b. $\mathbf{g}$, Percentage of monocyte IFN in immune cells in control mice $(n=3)$, APAP-treated $(n=4)$ and TAA-treated $(n=4)$ mice; significance was determined using a two-sided Wilcoxon test. Boxplots defined as in Fig. 2 b. h, GO term enrichment analysis of genes upregulated in monocyte IFN in comparison to Ly6C-positive monocytes. GO analysis was performed with GProfiler using standard settings; $P$ values shown are corrected for multiple hypothesis testing using the g:SCS algorithm. i, Transcription factor binding sites enriched in the promoters of genes upregulated in monocyte IFN in comparison to Ly6C-positive monocytes. Transcription factor binding site analysis was performed with GProfiler using standard settings; $P$ values shown are corrected for multiple hypothesis testing using the g:SCS algorithm. j, Diffusion maps explaining heterogeneity within Ly6C-positive monocytes. $\mathbf{k}$, Diffusion maps depicting expression of genes that change during the homing process. 
we noted a significant elevation of MYC in mice treated with APAP or TAA in comparison to controls (Fig. 4c, Extended Data Fig. 4c and source data for Extended Data Fig. 4c), while phosphorylated MYC remained unchanged (Extended Data Fig. 4d and source data for Extended Data Fig. 4c). Collectively, these data suggested that a common $M y c$-regulated program might commonly control the activation state of AAs, AAe and AAk during ALF.

MYC inhibition leads to amelioration of ALF. Given these results, we reasoned that MYC induction might contribute to the altered stellate, endothelial and Kupffer cell states described above, while inhibition of MYC transcriptional activity might potentially attenuate resident cellular response to ALF-induced signals. Such inhibition, including that of the upregulation of $\mathrm{Ccl} 2$, the key chemokine promoting monocyte recruitment, may also lead to an impairment in Ly6C-positive monocyte infiltration, thereby further contributing to attenuation of ALF-induced hepatic damage. To test our hypothesis, we induced ALF with APAP or TAA and cotreated mice with the MYC inhibitor KJ-Pyr-9 (ref. ${ }^{39}$ ) (henceforth, MYCi) and evaluated the impact of MYC inhibition on infiltration of Ly6C-positive
Neutrophil classica

Neutrophil proinflammatory

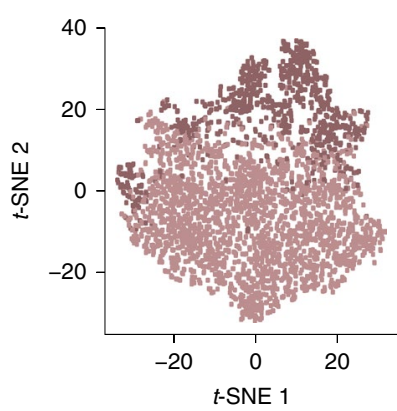

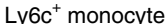
Monocyte IFN

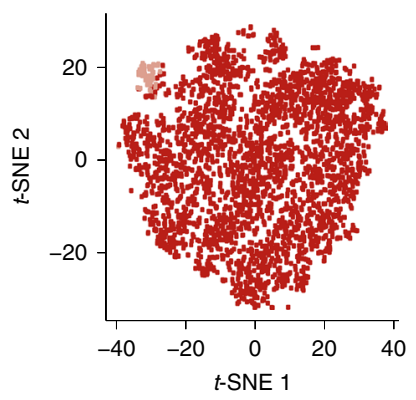

h

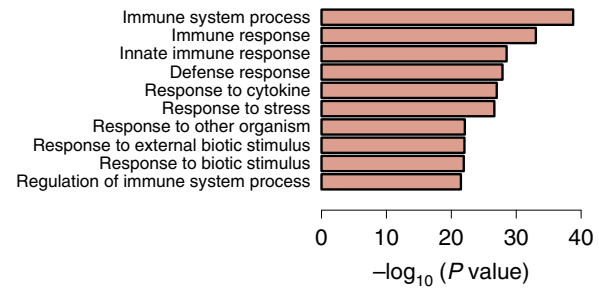

j

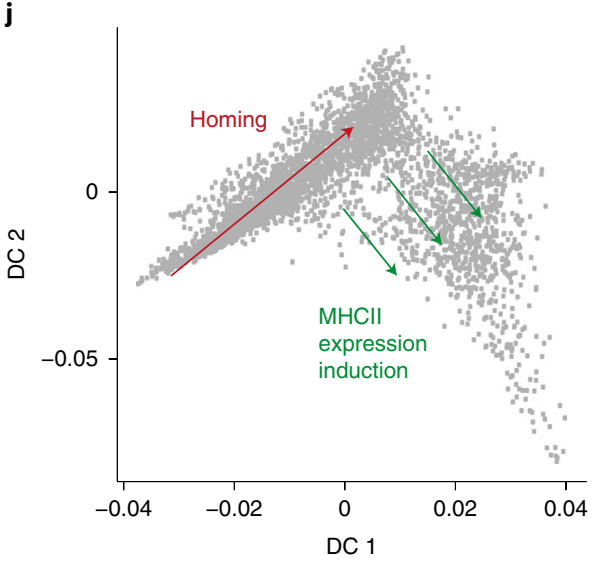

k
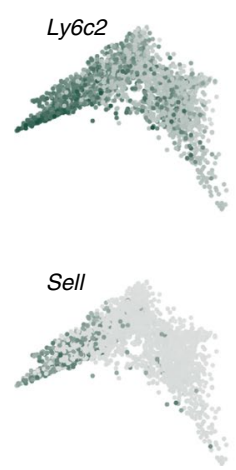

b
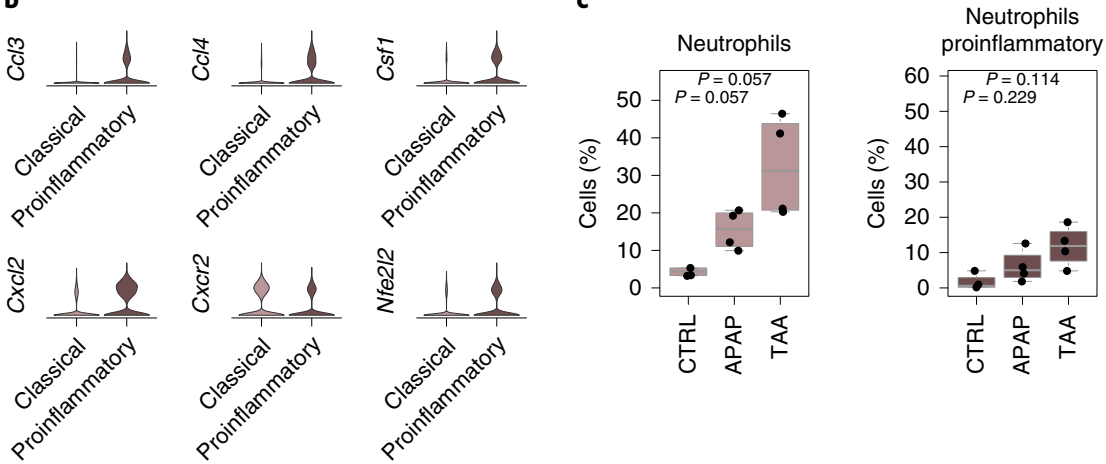

$\mathbf{f}$

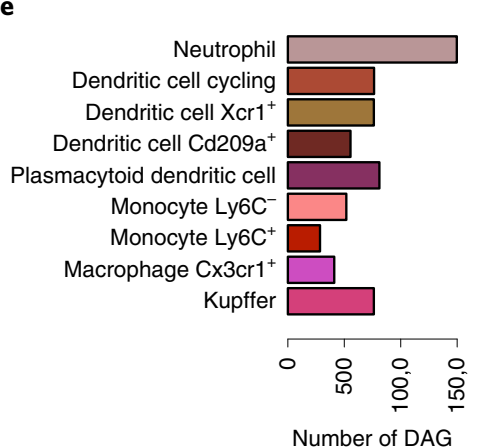

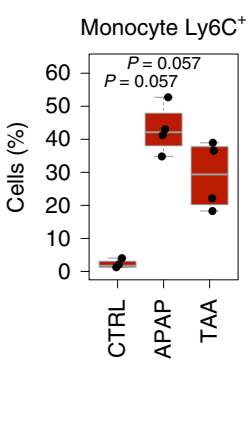

g

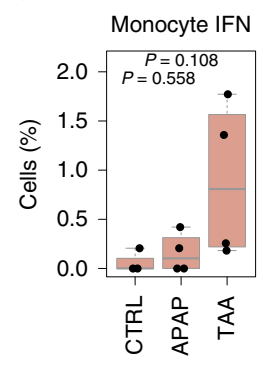

i

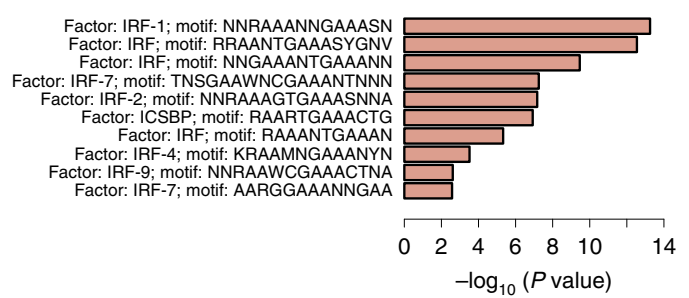

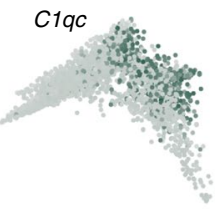
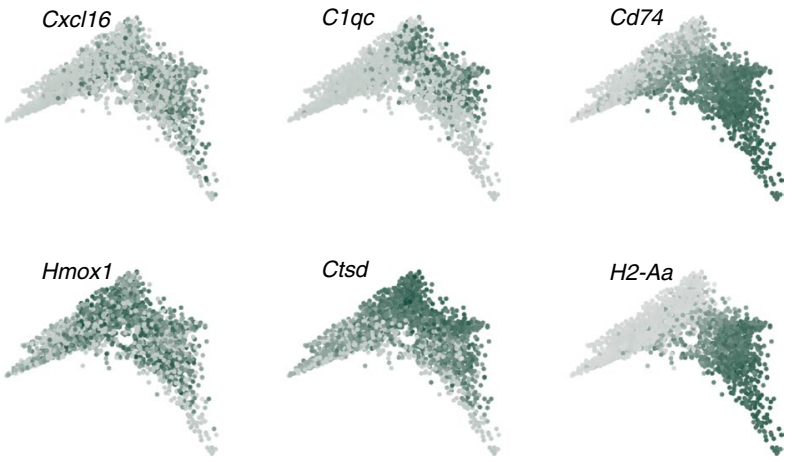
monocytes, serum levels of ALT and liver histology. Monocyte infiltration was assessed by flow cytometry as the fraction of F4/80 and Ly6C double-positive cells within the hepatic CD45-positive population (Extended Data Fig. 4e). Indeed, we observed a significant reduction in monocyte infiltration in mice induced with ALF and cotreated with the MYCi inhibitor, suggesting that MYC may play a role in induction of the inflammatory response to liver damage in this setting (Fig. 4d,e). Serum aspartate transaminase (AST) and alanine transaminase (ALT) activity in both ALF models (Fig. 4f,g), and mortality in APAP-administered mice (Fig. 4h), were likewise attenuated upon MYC inhibition. Histologically, hematoxylin and eosin (H\&E) staining of liver sections demonstrated, in both ALF models, that MYC inhibition led to reduced hepatic damage (Fig. 4i-k).

To corroborate these results, we performed single-cell RNA-seq in mice receiving MYCi in the APAP and TAA ALF models, or in the absence of acute hepatic insult, to examine the effect of MYC inhibition on liver-cell-specific gene expression patterns. In the absence of ALF, MYCi did not exert a notable effect on the gene expression landscape (Fig. 41 and Extended Data Figs. 4 f and 5). Closer examination, by differential expression analysis of pseudobulk counts between samples, revealed that, during steady state, MYC inhibition led to differential expression of 152 genes in stellate cells, 99 in Kupffer cells and 9 in endothelial cells. Gene Ontology analysis of differentially expressed genes in the presence of MYCi in stellate cells revealed downregulation of genes coding for ribosome proteins and other components of the translation machinery, coupled with upregulation of terms related to developmental processes which included, among others, genes from the AP1 family (Fos, Jun and Junb), as well as Col3a1 and Cxcl12 (Extended Data Fig. 6a). Similarly, in endothelial cells, MYC inhibition during steady state induced downregulation of AP1 family genes, while in Kupffer cells MYC inhibition drove downregulation of genes related to antigen processing and presentation (mainly MHCII genes) (Extended Data Fig. 6b-c).

Importantly, during ALF induction in the presence of $\mathrm{MYCi}$, activated populations (AAs, AAe and AAk) did not arise, and instead new cellular states of stellate, endothelial, Kupffer, dendritic and Tcells were observed, these being markedly different from the populations found in the absence of MYCi and did not cluster together with conventional activation states (Fig. 41 and Extended Data Fig. 4f). In addition, single-cell transcriptomic data reaffirmed that, in the presence of $\mathrm{MYCi}$, a near-total abrogation of hepatic Ly6C-positive monocyte infiltration was noted in both APAPand TAA-induced ALF (Extended Data Fig. 6d-e). Interestingly, neutrophil infiltration was not affected by MYCi (Extended Data Fig. 6d-e), probably explained by lack of downregulation of the main neutrophil chemoattractant $\mathrm{Cxcl} 2$, in contrast to marked suppression of the monocyte chemoattractant $C c l 2$ (Fig. 4m). Importantly, expression of the vast majority of the 77 genes constituting the common activation signature in activated stellate, Kupffer and endothelial cell subsets was markedly attenuated in MYCi-treated mice. Only two genes, metallothionein 1 and 2 ( $M t 1$ and $M t 2)$, remained unaffected by MYC inhibition, suggesting that expression of these oxidative stress-response genes is regulated by a different mechanism (Fig. 41 and Extended Data Fig. 6f-h).

Interestingly, MYC inhibition in ALF led to lower total gene expression. The strongest effect was observed in stellate cells, where the median number of detected transcripts dropped almost twofold, from 1,956 to 917. This effect was not a result of technical differences between samples, because the median number of detected transcripts in other cell types did not mirror such a difference (Extended Data Fig. 1d). One potential explanation for this reduction is apoptosis, which is associated with rapid messenger RNA decay and decrease in mitochondrial content ${ }^{40}$. Indeed, a strong downregulation of transcript number, with no increase in the percentage of mitochondrial reads, suggested that stellate cells may undergo cell death in the absence of MYC activity (Extended Data Fig. 3a). The process seemed to be specific to stellate cells, because endothelial and Kupffer cells did not exhibit these cell death hallmarks. Moreover, upregulation of the senescence and cell-cycle-arrest marker Cdkn1a (coding p21) in activated cells was attenuated compared to AAe and AAk in stellate cells upon MYC inhibition (Extended Data Fig. 6I). Gene Ontology analysis of genes upregulated in activated endothelial cells in the presence of MYC inhibition revealed terms related to apoptosis and its negative regulation and metabolism, while such analysis of Kupffer cells demonstrated mainly changes in immune-response-related terms (Extended Data Fig. 6j).

Microbiome modulation of the MYC program during ALF. We next sought to examine potential microbiome contributions to this ALF program. To this end, we induced disease in the APAP and TAA models following depletion of the microbiome of naïve or ALF-induced mice by a 2-week, wide-spectrum antibiotic treatment ( $\mathrm{ABX}, 1 \mathrm{gl}^{-1}$ ampicillin, neomycin, metronidazole and $0.5 \mathrm{gl}^{-1}$ vancomycin in drinking water $)^{41}$. To control for possible direct antibiotic impacts on liver physiology and ALF, we also induced ALF in germ-free mice (GF), which are devoid of a microbiome (Fig. 1a). Microbiome characterization by $16 \mathrm{~S}$ rRNA gene V4 region amplicon sequencing of colon and jejunum content during disease induction demonstrated no major differences in relative abundance, other than an increase in alpha diversity during ALF (Extended Data Fig. 7a-c).

Comparison of cell numbers in naïve and ALF-induced GF and SPF mice demonstrated no new distinct cell populations, but

Fig. 4 | Common activation signature of resident cells is regulated by MYC. a, Venn diagram showing overlap between sets of upregulated genes in Kupffer, stellate and endothelial cells. b. Transcription factor binding sites enriched in the promoters of a 77-gene common activation signature. Transcription factor binding site analysis was performed with GProfiler using standard settings; $P$ values shown are corrected for multiple hypothesis testing using the g:SCS algorithm. c, Quantification of MYC expression levels in healthy and ALF mice from immunoblots; control (CTRL), $n=20 ;$ APAP, $n=15$; TAA, $n=15$; significance was determined using a one-sided Wilcoxon test. Boxplot defined as in Fig. Fig. 2 . d-g, Cellular and enzymatic analysis of APAP and TAA liver failure models. $\mathbf{d}, \mathbf{e}$, FACS analysis of percentage of Ly6C-positive monocytes within all immune cells in the presence or absence of MYCi (APAP, d; TAA, e); significance was determined using a one-sided Wilcoxon test. APAP, $n=5$ for each group; TAA, $n=10$ for each group. Boxplots defined as in $\mathbf{b} . \mathbf{f}, \mathbf{g}$, Activity of AST and ALT in mouse serum in the presence or absence of MYCi (APAP, $\mathbf{f}$; TAA, $\mathbf{g}$ ); significance was determined using a one-sided Wilcoxon test. APAP, $n=10+5$ for each group from two independent experiments; TAA, $n=10$ for each group. Boxplots defined as in $b$. h, Survival curves for APAP-, APAP- and MYCi-treated mice; $n=15$ per group; significance was calculated using the survdiff function in the survival R package, which implements the Mantel-Haenszel test. i, Histology scores of H\&E-stained liver sections from the APAP liver failure model in the presence or absence of MYCi; significance was determined using a one-sided Wilcoxon test. $n=10+5$ for APAP and APAP + MYCi from two independent experiments; $n=5$ for CTRL and MYCi. j, Histology scores of H\&E-stained liver sections from TAA liver failure model in the presence or absence of MYCi; significance was determined using a one-sided Wilcoxon test. $n=10$ for each group. $\mathbf{k}$, Representative H\&E-stained liver sections. Scale bars, $100 \mu \mathrm{m}$. I, Balloon plot showing normalized and scaled expression of 77-gene common activation signature in the presence or absence of MYC inhibition in stellate, endothelial and Kupffer cells. MYCi $(n=2)$, APAP + MYCi $(n=2)$, TAA + MYCi $(n=2)$. m, Violin plots showing normalized and scaled expression of $\mathrm{C} / 2$ in stellate, endothelial and Kupffer cells and $\mathrm{CxCl} 2$ in Kupffer cells. 
a

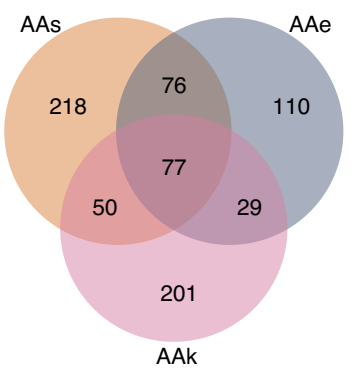

b

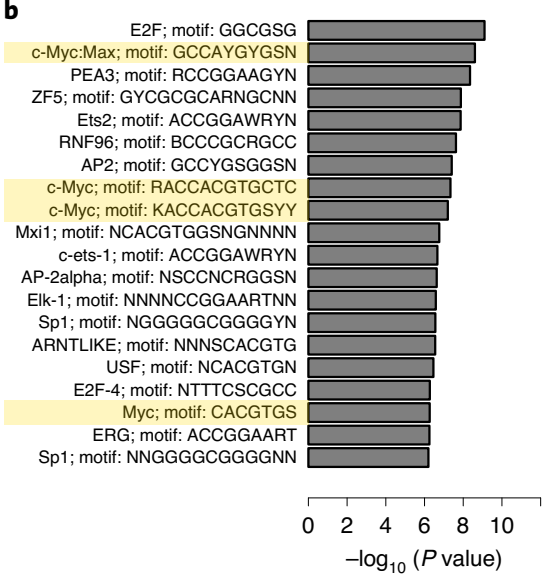

f

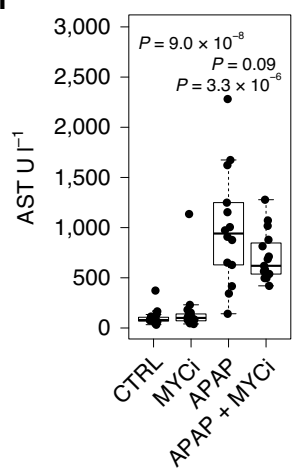

i

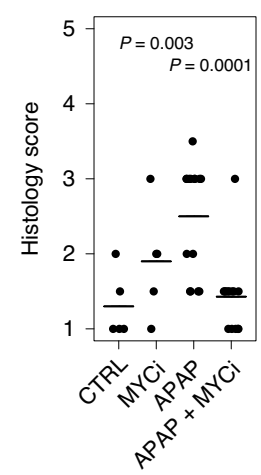

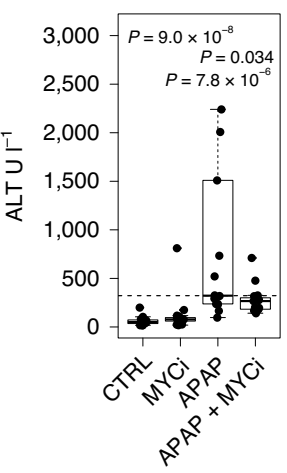

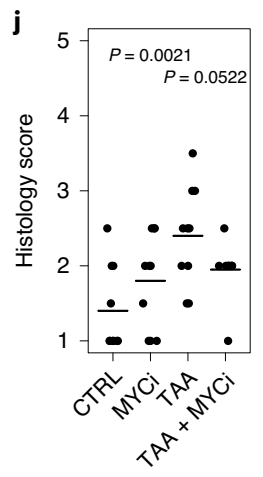

k c

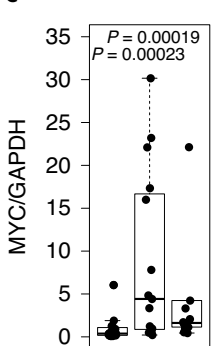

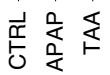

\section{d}

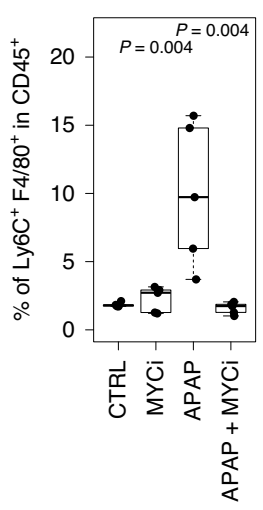

h

g
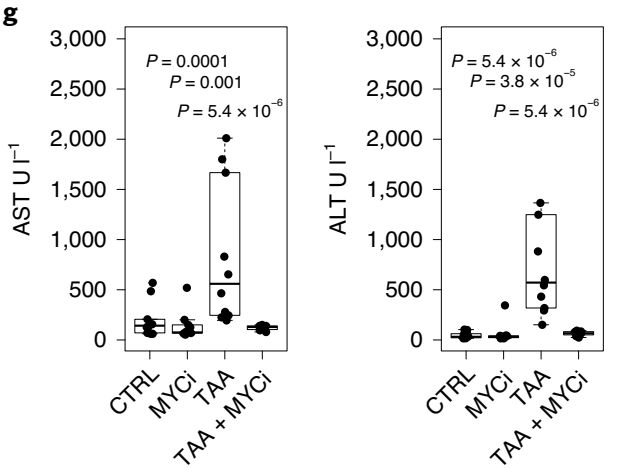

e

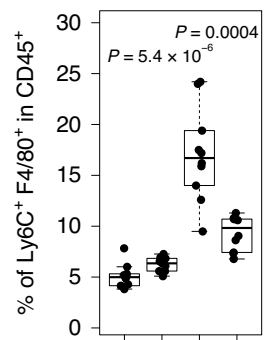

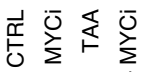
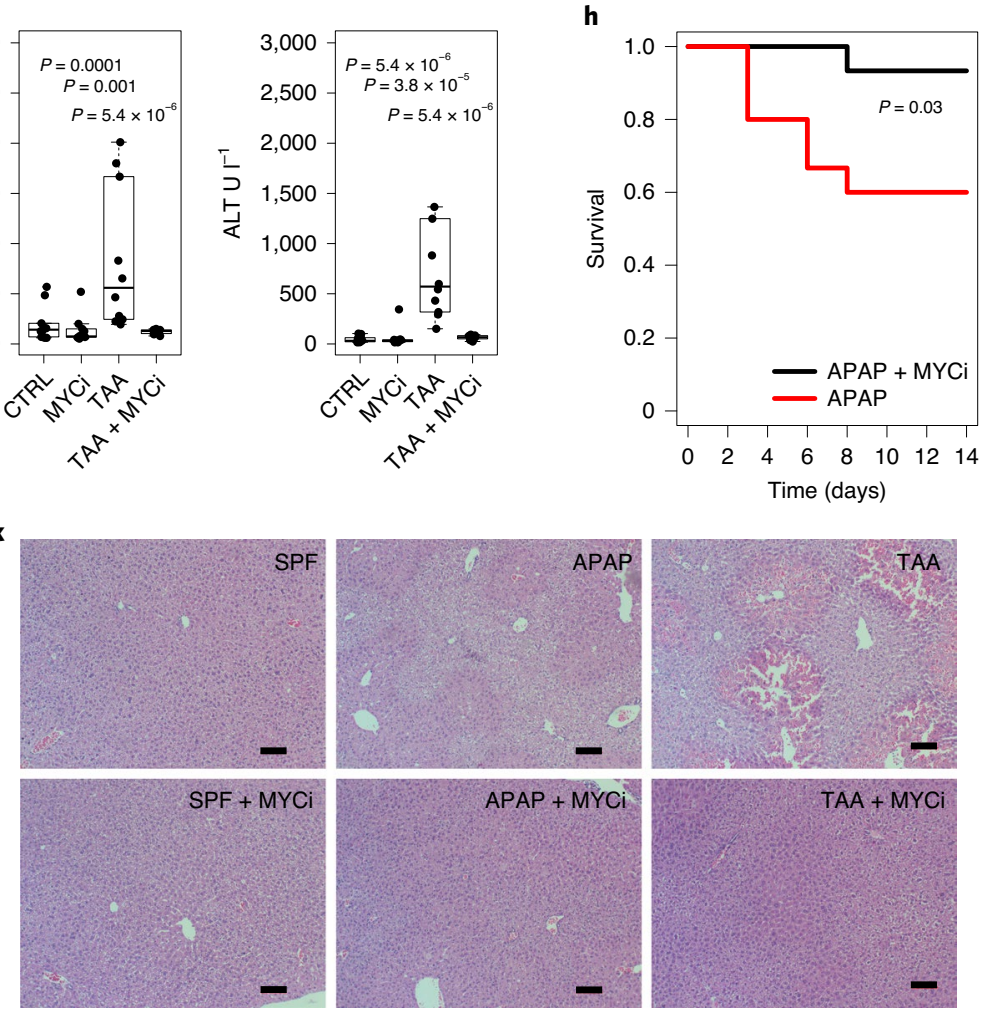

$\mathrm{APAP}+\mathrm{MYCi}$
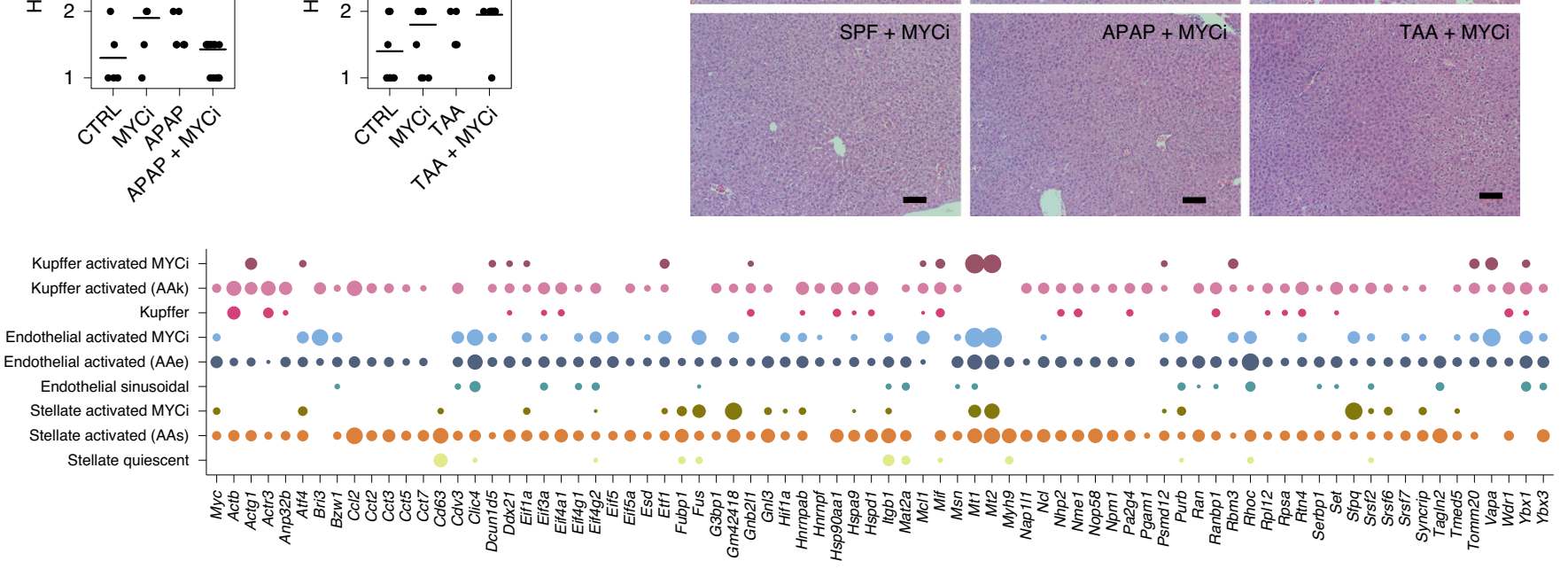

m
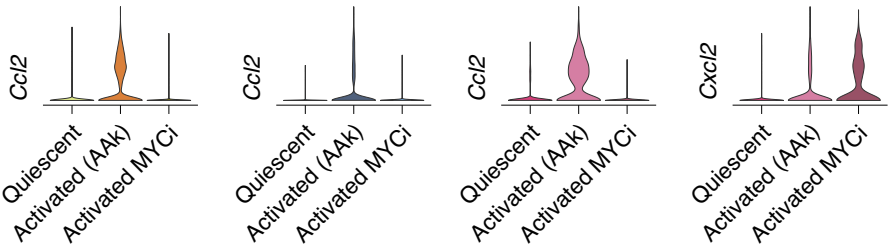
in both ALF models fewer AAs, AAe and AAk cells were noted upon microbiome depletion (Extended Data Fig. 7d). Importantly, APAP-induced ALF in GF mice was associated with a significantly reduced infiltration of Ly6C-positive monocytes compared to SPF mice, in agreement with the attenuated APAP-induced liver toxicity noted in GF mice ${ }^{42}$ (Fig. 5a). Moreover, liver damage in both the APAP and TAA models, as assessed by serum ALT and AST activity (Fig. 5b,c and Extended Data Figs. 4f and 8a) and histology (Fig. 5d,e), was milder in GF and ABX-treated mice as compared with microbiome-intact mice.

To determine whether functional differences in hepatic resident cells had contributed to this enhanced microbiome-induced monocyte infiltration, we next performed differential expression between GF and SPF mice using pseudobulk counts for each cell type with DESeq2. In naïve mice, the analysis revealed only two differentially expressed genes between SPF and GF mice-Cxcl14 in stellate cells and $\operatorname{Tr} f$ in cholangiocytes (DESeq2 false discovery rate (FDR) adjusted $P=3.65 \times 10^{-6}$ and $1.23 \times 10^{-6}$, respectively; Fig. 5f $)^{43}$. In contrast, comparison of differential gene expression between APAP-administered SPF and GF mice revealed 127 differentially expressed genes between AAs cells, 87 in AAe cells and 17 in AAk cells. We did not find differentially abundant genes between AAs, AAe and AAk cells in GF and SPF mice in the TAA model. Among the more abundant genes in APAP ALF-induced SPF mice were Ereg and Thbs 1 in stellate cells, Inhbb in endothelial cells and Cxcl2 in Kupffer cells (Fig. 5g) which, collectively, may have contributed to the difference in infiltration of Ly6C-positive monocytes between SPF and GF mice during ALF. Moreover, interleukin- 6 family members (Il6, Il11 and Lif) and activation markers such as Thbs1, Timp1, Cd44, Itga5, Ill7ra and Ereg featured higher expression levels in activated stellate cells in SPF mice, in agreement with the previously shown dependence of Ereg expression on the microbiome via TLR4 signaling in a hepatocellular carcinoma mouse model ${ }^{5}$. Under SPF conditions in AAe, we observed higher levels of activated endothelial cell markers, including $\operatorname{Lrg} 1$, Inhbb, Thbd and Bhlhe40, as compared to GF mice. Many stellate and endothelial cell genes upregulated in SPF mice were related to translation machinery. In Kupffer cells we found six genes to be upregulated in SPF mice, including Marco and
Cxcl2. Only a few genes in GF mice were expressed at higher levels than in SPF mice-for example, Cox17 and Comt in Kupffer cells (Extended Data Fig. 8b-e). Depletion of the microbiome with antibiotics led to similar gene expression changes that were intermediate in extent between those for GF and SPF (Fig. 5b-g). Importantly, these observed microbiome gene-expression effects raised the possibility that the entire MYC-regulated ALF signature may be affected by the microbiome. Indeed, mean expression of the MYC-regulated gene signature in stellate, endothelial and Kupffer cells was significantly higher in SPF than in GF and ABX-treated mice (Fig. 5h). Together, these results suggest that microbiome-mediated upstream signals may regulate MYC during ALF.

TLR signaling and downstream adapters are necessary for activation of the MYC program in ALF. We hypothesized that the microbiome regulates the MYC program in AAs, AAe and AAk cells during ALF through triggering of TLR signaling. In this scenario, signaling by damage-associated molecular patterns (DAMPs) originating from damaged liver cells, coupled with portal venous microbial-associated molecular patterns (MAMPs) originating from the gut microbiome, jointly drive TLR-induced MYC activation in these cells, leading to downstream immune cell infiltration and exacerbated disease. Indeed, a reporter cell assay (Methods) identified portal vein TLR2, TLR4, TLR5, TLR9, NOD1 and NOD2 agonists upon induction of TAA ALF, and TLR4, TLR9 and NOD2 agonists upon induction of APAP ALF (Extended Data Fig. 9).

To test whether potential TLR involvement exists downstream of these MAMPs, we utilized MyD88-Trif double-knockout (MyD88-Trif dKO) mice, which lack both adapter proteins necessary for TLR signaling, and performed single-cell RNA-seq under both naïve and APAP-treated conditions in these mice and compared to wild-type (WT) controls. In steady state, all cellular states in MyD88-Trif dKO mice were similar to WT mice, except for MyD88-Trif dKO Kupffer cells, which clustered separately from the respective cells in WT mice (Extended Data Figs. 5 and 10a). Interestingly, MyD88-Trif dKO Kupffer cells featured higher expression of interferon-responsive factors as compared to WT Kupffer cells (Extended Data Fig. 10b).

Fig. 5 | The microbiome modulates response to acute insult via MYC and TLR. a, FACS analysis of percentage of Ly6C-positive monocytes within all immune cells in GF and SPF mice; significance was determined using a one-sided Wilcoxon test; $n=10$ for each group. b,c, Activity of AST and ALT in mouse serum from APAP (b) and TAA (c) liver failure models in GF, ABX and SPF mice; significance was determined using a one-sided Wilcoxon test. APAP, $n=10+10$ for ABX and SPF and $n=10+9$ for GF, from two independent experiments; TAA, $n=10$ for each group. Boxplots defined as in Fig. 2 b. d,e, Histology scores of H\&E-stained liver sections from APAP (d) and TAA (e) liver failure models in GF, ABX and SPF mice; significance was determined using a one-sided Wilcoxon test. APAP, $n=10+10$ for ABX and SPF and $n=10+9$ for GF, from two independent experiments; TAA, $n=10$ for each group. f, Violin plots showing normalized and scaled expression of genes differentially expressed between healthy GF and SPF mice: Cxcl14 in stellate cells and Trf in cholangiocytes. $\mathbf{g}$, Violin plots showing normalized and scaled expression of examples of common genes in three activated cell types that differentially expressed between GF and SPF conditions. $\mathbf{h}$, Expression of the 77-gene common activation signature in GF, ABX and SPF mice in activated resident cell types. Significance was calculated using a one-sided paired Wilcoxon test. GF $+\operatorname{APAP}(n=2, c S=474, c E=836, c K=65) ; A B X+A P A P(n=2, c S=509$, $\mathrm{cE}=187, \mathrm{cK}=27)$; SPF + APAP ( $n=4, \mathrm{cS}=4,339, \mathrm{cE}=1,517, \mathrm{cK}=265)$; boxplots defined as in Fig. 2b. i-k, Violin plots showing normalized and scaled expression of $\mathrm{Ccl} 2$ and $\mathrm{Mt}$ in activated stellate cells (i), activated endothelial cells (j) and activated Kupffer cells in WT mice ( $\mathbf{k}$ ) in the presence of MYCi, and in MyD88-Trif knockout (KO) mice. I, Boxplots showing pseudobulk transcripts per million (TPM) of the 77-gene common activation signature in WT mice: $\operatorname{SPF}(n=3, c S=1,999, c E=1,463, c K=659), S P F+\operatorname{APAP}(n=4, c S=4,339, c E=1,517, c K=265)$ and SPF $+\mathrm{TAA}(n=4, c S=910, \mathrm{cE}=1,456, \mathrm{cK}=285)$ in the presence of MYCi: MYCi $(n=2, c S=906, c E=851, c K=554), S P F+A P A P+M Y C i(n=2, c S=251, c E=303, c K=125)$ and SPF + TAA + MYCi $(n=2, c S=198, c E=512, c K=233)$ and in MyD88-Trif $K O$ mice: MyD88-Trif $K O(n=2, c S=1,381, c E=1,721, c K=245), M y D 88-T r i k ~ K O+A P A P(n=2$, $\mathrm{cS}=1,556, \mathrm{cE}=1,463, \mathrm{cK}=349)$. Significance was calculated using a two-sided paired Wilcoxon test. ${ }^{\star \star \star} P<0.001 ;$ boxplots defined as in Fig. $2 \mathrm{~b}$. $P$ values in stellate cells: SPF versus SPF + APAP. $5.065 \times 10^{-13} ;$ SPF versus SPF + TAA, 9.656 $\times 10^{-12}$; SPF + APAP versus SPF + APAP + MYCi, 1.135 $\times 10^{-15} ;$ SPF + TAA versus SPF + TAA + MYCi, $2.662 \times 10^{-14}$; MyD88-Trif KO versus MyD88-Trif KO + APAP, 0.243; SPF + APAP versus MyD88-Trif KO + APAP, 9.914×10-11; in endothelial cells: SPF versus SPF + APAP, 4.473 $\times 10^{-11}$; SPF versus SPF + TAA, 6.746 $\times 10^{-10}$; SPF + APAP versus SPF + APAP + MYCi, 5.196 $\times 10^{-6}$; SPF + TAA versus SPF + TAA + MYCi, 4.935×10-6; MyD88-Trif KO versus MyD88-Trif KO + APAP, 0.117; SPF + APAP versus MyD88-Trif KO + APAP, $8.52 \times 10^{-4}$; in Kupffer cells: SPF versus SPF + APAP, $5.984 \times 10^{-11} ;$ SPF versus SPF + TAA, $1.461 \times 10^{-8} ;$ SPF + APAP versus SPF + APAP + MYCi, $1.287 \times 10^{-8}$; SPF + TAA versus SPF + TAA + MYCi, 1.517×10-7; MyD88-Trif KO versus MyD88-Trif KO + APAP, 0.236; SPF + APAP versus MyD88-Trif KO + APAP, $2.739 \times 10^{-4} \cdot \mathbf{m}, \mathbf{n}$, Barplots showing infiltration of Ly6C-positive monocytes ( $\mathbf{m}$ ) and neutrophils (n) in the presence or absence of MYCi and in MyD88-Trif $\mathrm{KO}$ mice; different colors of the bars of neutrophils denote subpopulations. $n$, number of mice, cS, number of stellate cells, $c E$, number of endothelial cells, cK, number of Kupffer cells. 
a

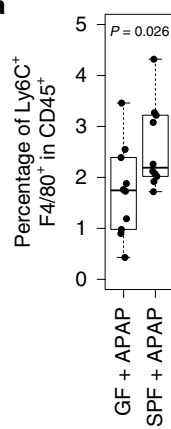

b

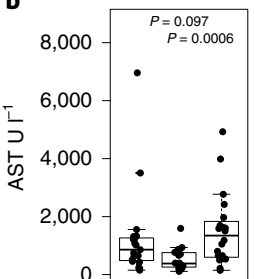

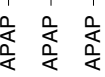

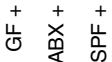

e

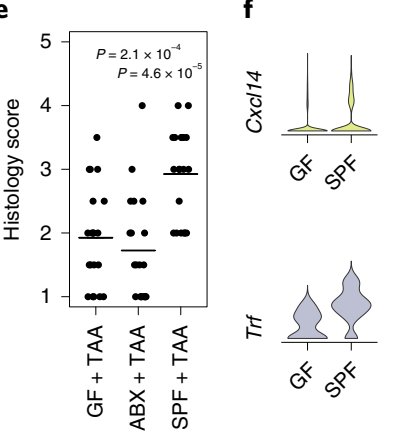

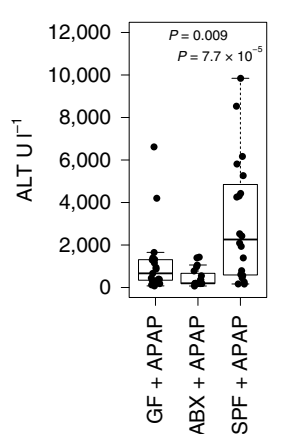

g

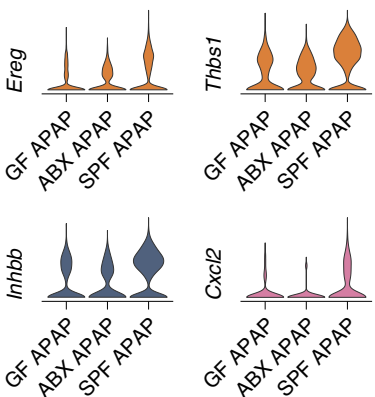

c

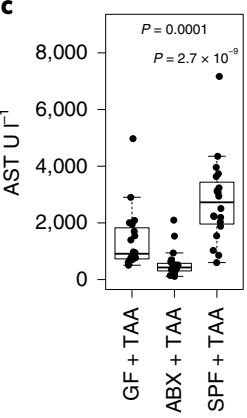

h

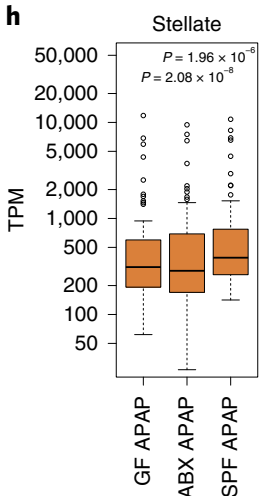

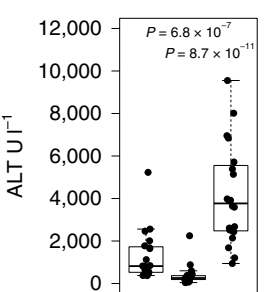

发委事

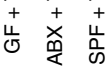

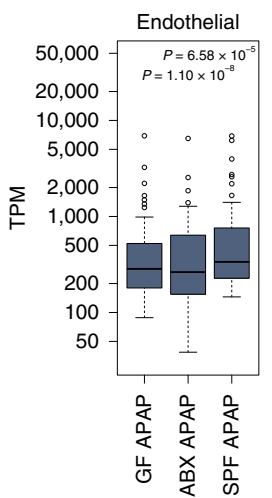

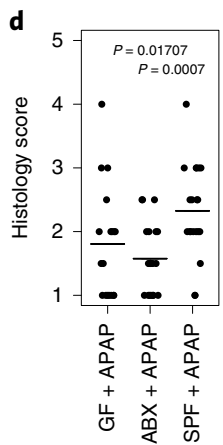

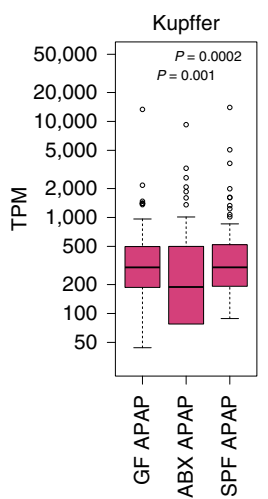

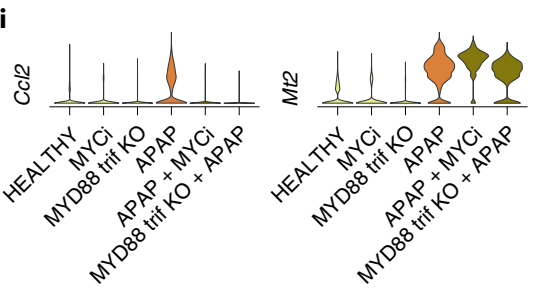

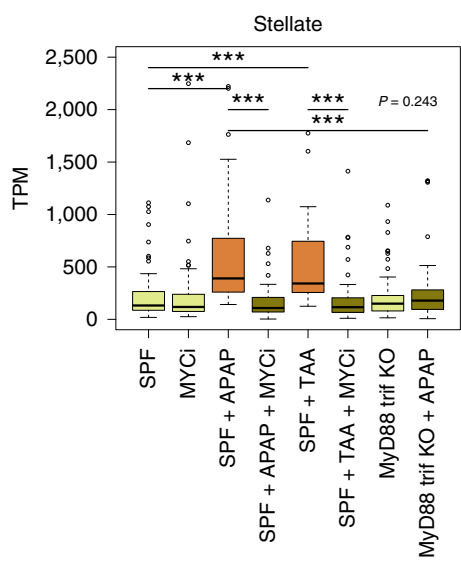

j
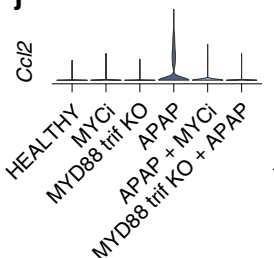

$211+1<$

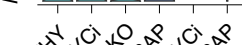

k
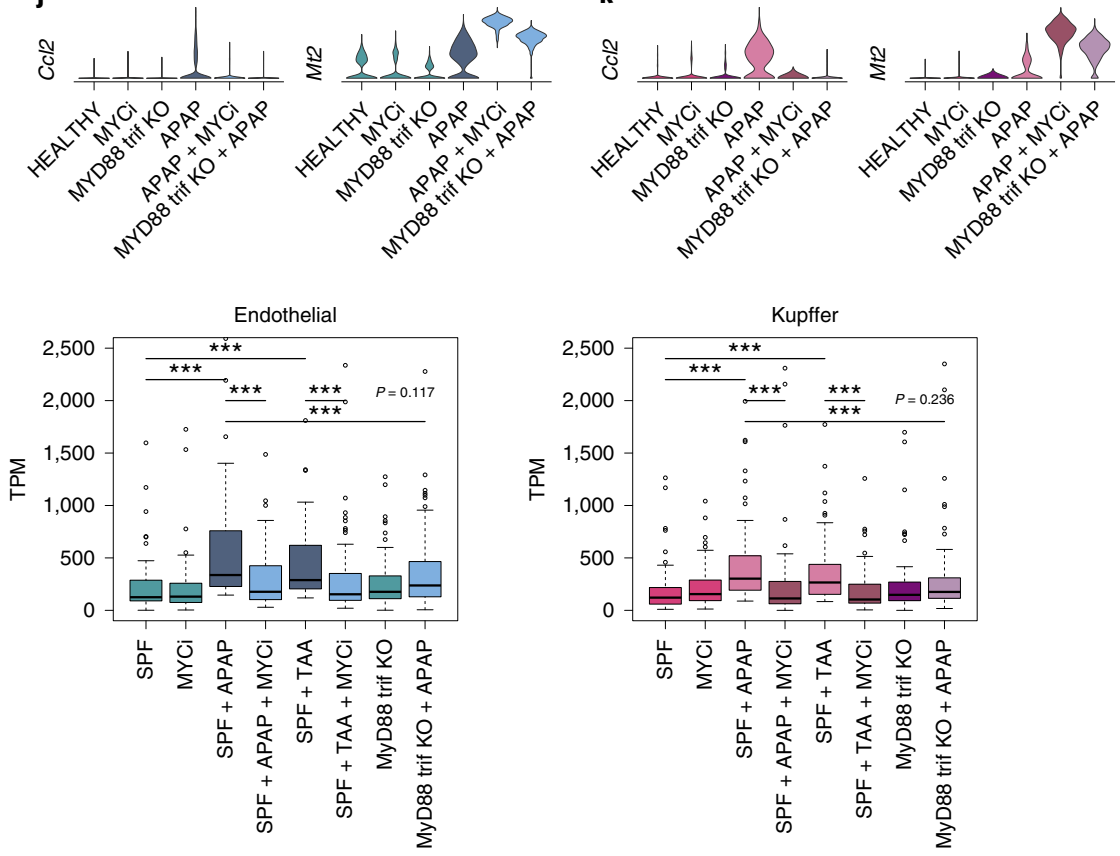

m

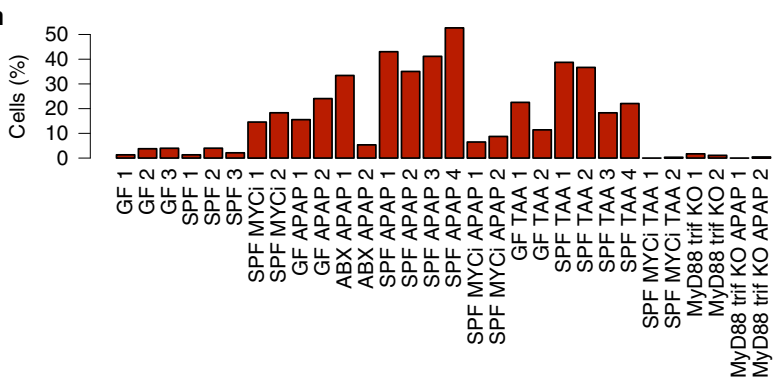

n

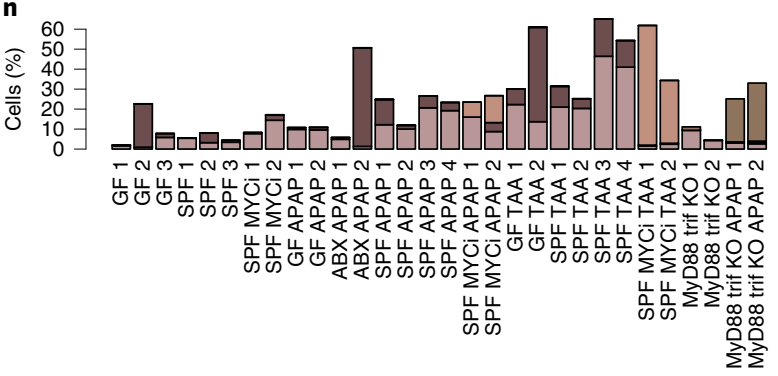




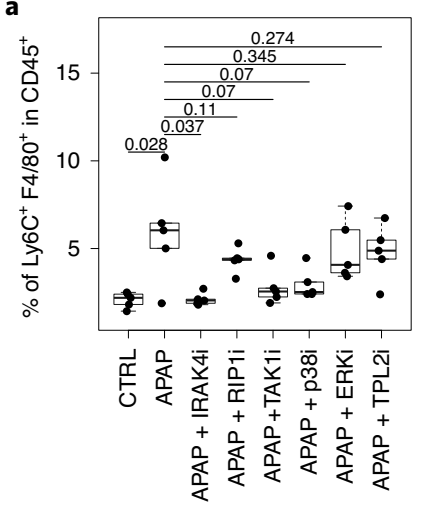

b

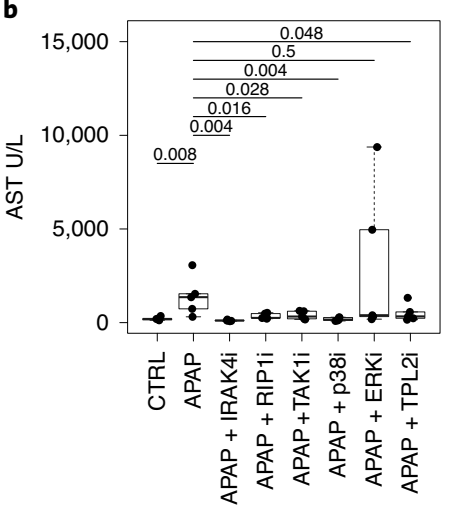

c

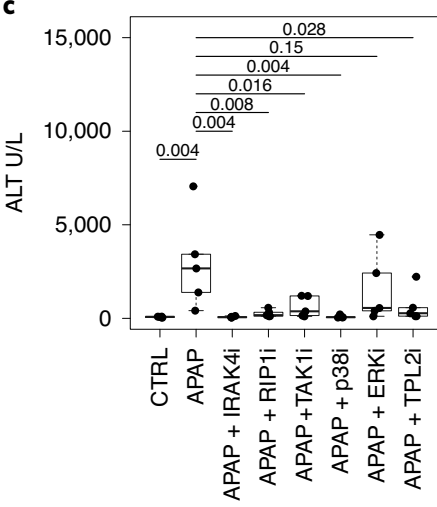

d

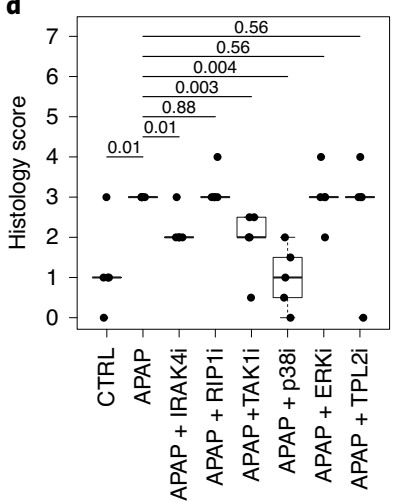

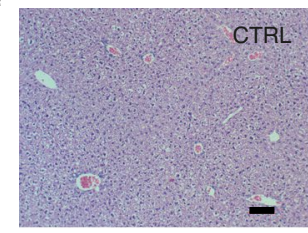

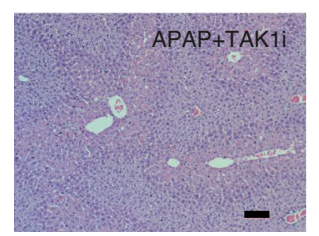

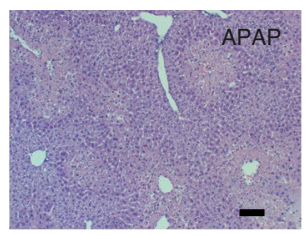

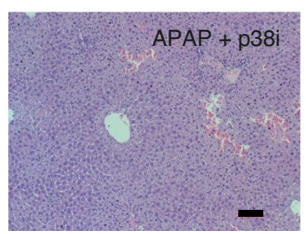

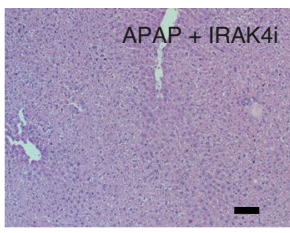

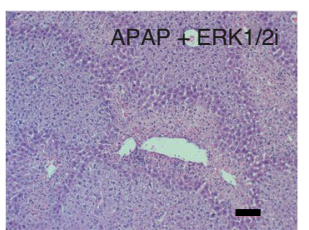

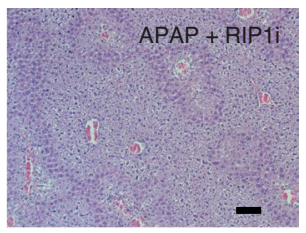

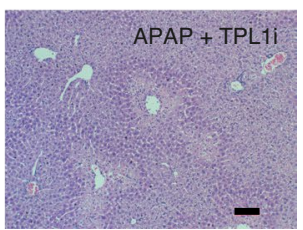

f

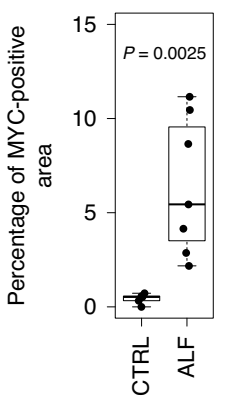

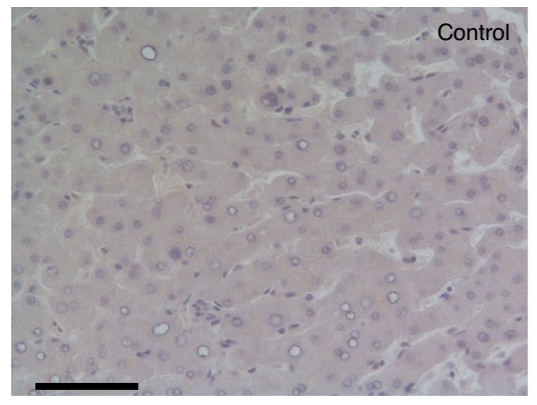
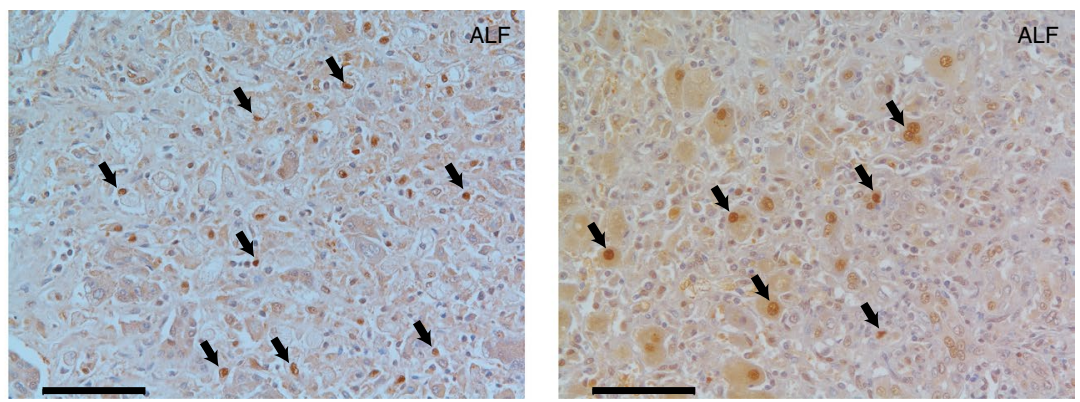

Fig. 6 | The MAPK pathway relays signaling from TLR to MYC. a, FACS analysis of infiltration of Ly6C-positive monocytes, shown as a percentage of all immune cells from mice receiving APAP and inhibitors of MAPK pathway proteins; significance was determined using a one-sided Wilcoxon test; $n=5$ for each group. b,c, Serum activity of AST (b) and ALT (c) for mice receiving APAP and inhibitors of MAPK pathway proteins; significance was determined using a one-sided Wilcoxon test; $n=5$ for each group. d,e, Histology scores (d) of H\&E-stained liver sections from APAP liver failure model (e); significance was determined using a one-sided Wilcoxon test; $n=5$ for each group. Boxplot in a-d defined as in Fig. $2 b$; numbers above plots indicate $P$ values. f, Immunohistochemistry of MYC in human controls and ALF samples; $n=5$ for controls and $n=7$ for ALF. Boxplot defined as in Fig. $2 b ;$ significance was calculated using a two-sided Wilcoxon test. $\mathbf{g}$, Examples of MYC immunohistochemistry. Arrows indicate positive staining for MYC. All scale bars, $100 \mu$ m.

Importantly, upon induction of APAP-driven ALF, MyD88-Trif dKO stellate and endothelial cells become aberrantly activated, assuming a transcriptional state distinct from that of ALF-induced WT mice and nearly identical to that of MYCi-treated mice (Extended Data Fig. 5). MyD88-Trif dKO Kupffer cells also became aberrantly activated, but their activation state was distinct from that of both APAP-induced and MYCi-treated, APAP-induced WT mice (Extended Data Fig. 10b). Similarly, neutrophils assumed an activated pattern markedly different from that observed in ALF-induced WT controls (Extended Data Figs. $4 \mathrm{f}$ and 10a). In corroboration of these findings, stellate, endothelial and Kupffer cells in MyD88-Trif dKO mice expressed metallothioneins $M t 1$ and $M t 2$ in response to APAP but failed to express Ccl2, Ccl7, Acta2, Csf1 and Inhbb, similarly to MYCi-treated mice in which MYC transcriptional activity was inhibited (Fig. 5i-k and Extended Data Fig. 10c-e).
Furthermore, expression of the 77-gene MYC-induced 'signature' in stellate, endothelial and Kupffer cells was significantly attenuated in MyD88-Trif dKO mice as compared to WT littermate controls, similarly to that observed upon MYCi treatment (Fig. 51). Moreover, ALF-induced monocyte infiltration was blocked in the absence of TLR signaling in MyD88-Trif dKO mice while that of neutrophils remained unaffected. (Fig. $5 \mathrm{~m}, \mathrm{n}$ ).

We next sought to study the downstream events by which TLR activation during ALF leads to activation of the MYC program during ALF. One apparent candidate pathway is MAPK, relaying signals from TLRs sensing MAMPs and DAMPs to regulate downstream MYC-dependent gene expression. In support of such pathway involvement in ALF are observations suggesting that TLR4 signaling regulates microbiota-dependent Ereg expression in hepatocellular carcinoma in stellate cells ${ }^{5}$, the senescence-associated secretory 
phenotype being downregulated in the absence of TLR2 (ref. ${ }^{44}$ ), and the strong induction of Map3k8 expression (coding for TPL2) noted in the presence of MYCi during ALF (Extended Data Fig. 10f). To test for MAPK pathway involvement in ALF, we selected six proteins from the pathway for which small molecule inhibitors are available: IRAK4, RIP1, TAK1, TPL2, ERK1/2 and p38, and tested them in APAP-induced ALF ${ }^{45-50}$. Indeed, we observed significant reduction of monocyte infiltration in mice receiving inhibitors of IRAK4, TAK1 and p38 (Fig. 6a). AST activity in serum was significantly lower in the presence of IRAK4, RIP1, TAK1 and p38, while liver-specific ALT activity was lower in the presence of IRAK4, RIP1 and p38 (Fig. 6b,c). Histopathological analysis reaffirmed these results, demonstrating significantly reduced liver damage in mice subjected to IRAK4, TAK1 or p38 inhibition (Fig. 6d,e). Importantly, ERK1/2 inhibition did not induce any trend towards lower ALF severity, suggesting that it may not be involved in the observed regulation. Together, these results suggest that the ALF MYC program in stellate, endothelial and Kupffer cells is regulated via upstream TLR signaling, probably activated by tissue damageand microbiome-associated DAMPs and MAMPs. Hepatic TLR signaling, in turn, regulates MYC via activation of the MAPK pathway. TLR and MAPK pathway inhibition, or microbiome depletion, induces marked suppression of this cell-specific MYC program, thereby driving a significant attenuation of ALF.

MYC is upregulated in human ALF. Finally, we aimed to determine whether the noted MYC involvement in animal models of ALF could be observed in human patients. To this end, we quantified by immunohistochemistry the levels of MYC in hepatic liver sections obtained from seven patients with ALF (Extended Data Fig. 10g). As 'healthy' controls we used liver samples obtained from five cadaveric liver donors (Extended Data Fig. 10g). Indeed, a significant increase in nuclear MYC protein levels was noted in patients with ALF as compared to controls (Fig. 6f,g). The functional implication of this MYC upregulation, and whether inhibition of MYC signaling by the above-mentioned checkpoints may impact the course and outcome of human ALF, merit further studies.

\section{Discussion}

In this work, using two ALF animal models we uncovered new HSC, LSEC and myeloid cellular states characterized by distinct transcriptional signatures. We suggest that, during ALF, both microbiota-derived MAMPs and necrosis-derived DAMPs ${ }^{51}$ signal to TLRs in resident stellate, sinusoidal endothelial and Kupffer cells, which activate MYC through IRAK4- and p38-dependent singaling $^{52,53}$. Activated MYC in these cells, in turn, impacts downstream gene expression, leading to liver infiltration of Ly6C-positive monocytes. Importantly, MYC inhibition prevented activation of these ALF-associated subsets, thereby leading to significant amelioration of liver damage.

Our findings may constitute a first step towards the identification of new therapeutic targets in human ALF. Currently, beside liver transplantation, intravenous $\mathrm{N}$-acetylcysteine constitutes the sole APAP-induced ALF treatment, by replenishing glutathione reserves depleted in APAP detoxification. This intervention is only partially effective and is accompanied by adverse effects, including anaphylactoid reactions in as many as $15 \%$ of cases. Even fewer therapeutic options are available for other ALF etiologies. Identification of MYC signaling as a potential regulatory axis of cellular response to ALF may enable disruption of ALF-induced liver pathology and damage, and merits further studies in human patients.

Future studies utilizing other ALF models in murine and human ALF should assess the commonalities and distinctions between cellular subsets and gene expression profiles in different ALF entities, and determine these cellular and genomic dynamics during hepatic regeneration from ALF. For example, the inflammasome-IL1 signaling axis may constitute another potential MyD88-MAPK-MYC-dependent avenue of regulation of inflammation and merits further studies. Future research may evaluate the context-specific contribution of distinct commensals and their products to the MYC-dependent gene signature. With these limitations notwithstanding, we suggest that cell- and pathway-specific molecular elucidation of ALF may allow utilization of host and microbiome inhibitors of signaling (such as MYCi and P38 inhibitors, researched in the cancer context) as future interventions in ALF.

\section{Online content}

Any methods, additional references, Nature Research reporting summaries, source data, extended data, supplementary information, acknowledgements, peer review information; details of author contributions and competing interests; and statements of data and code availability are available at https://doi.org/10.1038/ s41591-020-1102-2.

Received: 26 November 2019; Accepted: 9 September 2020; Published online: 26 October 2020

\section{References}

1. Bernal, W. \& Wendon, J. Acute liver failure. N. Engl. J. Med. 369, 2525-2534 (2013).

2. Hinson, J. A., Roberts, D. W. \& James, L. P. Mechanisms of acetaminopheninduced liver necrosis. Handb. Exp. Pharmacol. 196, 369-405 (2010).

3. Jaeschke, H. Reactive oxygen and mechanisms of inflammatory liver injury: present concepts. J. Gastroenterol. Hepatol. 26, 173-179 (2011).

4. Andrew Clayton, T. et al. Pharmaco-metabonomic phenotyping and personalized drug treatment. Nature 440, 1073-1077 (2006).

5. Dapito, D. H. et al. Promotion of hepatocellular carcinoma by the intestinal microbiota and TLR4. Cancer Cell 21, 504-516 (2012).

6. Rühlemann, M. C. et al. Faecal microbiota profiles as diagnostic biomarkers in primary sclerosing cholangitis. Gut 66, 753-754 (2017).

7. Henao-Mejia, J. et al. Inflammasome-mediated dysbiosis regulates progression of NAFLD and obesity. Nature 482, 179-185 (2012).

8. Llopis, M. et al. Intestinal microbiota contributes to individual susceptibility to alcoholic liver disease. Gut 65, 830-839 (2016).

9. Hajovsky, H. et al. Metabolism and toxicity of thioacetamide and thioacetamide S-oxide in rat hepatocytes. Chem. Res. Toxicol. 25, 1955-1963 (2012).

10. Woolbright, B. et al. The impact of sterile inflammation in acute liver injury. J. Clin. Transl. Res. 3, 170-188 (2017).

11. Mederacke, I., Dapito, D. H., Affò, S., Uchinami, H. \& Schwabe, R. F. High-yield and high-purity isolation of hepatic stellate cells from normal and fibrotic mouse livers. Nat. Protoc. 10, 305-315 (2015).

12. Heng, T. S. P. \& Painter, M. W., Immunological Genome Project Consortium. The Immunological Genome Project: networks of gene expression in immune cells. Nat. Immunol. 9, 1091-1094 (2008).

13. Strauss, O., Phillips, A., Ruggiero, K., Bartlett, A. \& Dunbar, P. R. Immunofluorescence identifies distinct subsets of endothelial cells in the human liver. Sci. Rep. 7, 44356 (2017).

14. Kalucka, J. et al. Single-cell transcriptome atlas of murine endothelial cells. Cell 180, 764-779 (2020).

15. Yang, C.-Y. et al. CLEC4F is an inducible C-type lectin in F4/80-positive cells and is involved in alpha-galactosylceramide presentation in liver. PLOS ONE 8, e65070 (2013)

16. Krenkel, O. \& Tacke, F. Liver macrophages in tissue homeostasis and disease. Nat. Rev. Immunol. 17, 306-321 (2017).

17. MacParland, S. A. et al. Single cell RNA sequencing of human liver reveals distinct intrahepatic macrophage populations. Nat. Commun. 9, 4383 (2018).

18. Cavalli, M. et al. A multi-omics approach to liver diseases: integration of single nuclei transcriptomics with proteomics and HiCap bulk data in human liver. OMICS 24, 180-194 (2020).

19. Ramachandran, P. et al. Resolving the fibrotic niche of human liver cirrhosis at single-cell level. Nature 575, 512-518 (2019).

20. Krenkel, O., Hundertmark, J., Ritz, T. P., Weiskirchen, R. \& Tacke, F. Single cell RNA sequencing identifies subsets of hepatic stellate cells and myofibroblasts in liver fibrosis. Cells 8, 503 (2019).

21. Reynaert, H., Thompson, M. G., Thomas, T. \& Geerts, A. Hepatic stellate cells: role in microcirculation and pathophysiology of portal hypertension. Gut 50, 571-581 (2002).

22. Schmidt-Arras, D. \& Rose-John, S. IL-6 pathway in the liver: from physiopathology to therapy. J. Hepatol. 64, 1403-1415 (2016).

23. Maeshima, K. et al. A protective role of interleukin 11 on hepatic injury in acute endotoxemia. Shock 21, 134-138 (2004). 
24. Campisi, J. \& d'Adda di Fagagna, F. Cellular senescence: when bad things happen to good cells. Nat. Rev. Mol. Cell Biol. 8, 729-740 (2007).

25. Coppé, J.-P., Desprez, P.-Y., Krtolica, A. \& Campisi, J. The senescenceassociated secretory phenotype: the dark side of tumor suppression. Annu. Rev. Pathol. 5, 99-118 (2010)

26. Poisson, J. et al. Liver sinusoidal endothelial cells: physiology and role in liver diseases. J. Hepatol. 66, 212-227 (2017).

27. Shaulian, E. \& Karin, M. AP-1 as a regulator of cell life and death. Nat. Cell Biol. 4, E131-E136 (2002).

28. Karlmark, K. R., Wasmuth, H. E., Trautwein, C. \& Tacke, F. Chemokinedirected immune cell infiltration in acute and chronic liver disease. Expert Rev. Gastroenterol. Hepatol. 2, 233-242 (2008).

29. Fabregat, I. et al. TGF- $\beta$ signalling and liver disease. FEBS J. 283, 2219-2232 (2016)

30. Ramilowski, J. A. et al. A draft network of ligand-receptor-mediated multicellular signalling in human. Nat. Commun. 6, 7866 (2015).

31. Dambach, D. M., Watson, L. M., Gray, K. R., Durham, S. K. \& Laskin, D. L. Role of CCR2 in macrophage migration into the liver during acetaminophen-induced hepatotoxicity in the mouse. Hepatology 35, 1093-1103 (2002).

32. Huebener, P. et al. The HMGB1/RAGE axis triggers neutrophil-mediated injury amplification following necrosis. J. Clin. Invest. 125, 539-550 (2019).

33. McDonald, B. et al. Intravascular danger signals guide neutrophils to sites of sterile inflammation. Science 330, 362-366 (2010).

34. Silvestre-Roig, C., Hidalgo, A. \& Soehnlein, O. Neutrophil heterogeneity: implications for homeostasis and pathogenesis. Blood 127, 2173-2181 (2016)

35. Mayadas, T. N., Cullere, X. \& Lowell, C. A. The multifaceted functions of neutrophils. Annu. Rev. Pathol. 9, 181-218 (2014).

36. Nguyen, T., Nioi, P. \& Pickett, C. B. The Nrf2-antioxidant response element signaling pathway and its activation by oxidative stress. J. Biol. Chem. $\mathbf{2 8 4}$ 13291-13295 (2009)

37. Brempelis, K. J. \& Crispe, I. N. Infiltrating monocytes in liver injury and repair. Clin. Transl. Immunol. 5, e113 (2016).

38. Angerer, P. et al. destiny: diffusion maps for large-scale single-cell data in R. Bioinformatics 32, 1241-1243 (2016)

39. Hart, J. R. et al. Inhibitor of MYC identified in a Kröhnke pyridine library. Proc. Natl Acad. Sci. USA 111, 12556-12561 (2014).
40. Thomas, M. P. et al. Apoptosis triggers specific, rapid, and global mRNA decay with 3' uridylated intermediates degraded by DIS3L2. Cell Rep. 11, 1079-1089 (2015)

41. Elinav, E. et al. NLRP6 inflammasome regulates colonic microbial ecology and risk for colitis. Cell 145, 745-757 (2011).

42. Thaiss, C. A. et al. Microbiota diurnal rhythmicity programs host transcriptome oscillations. Cell 167, 1495-1510.e12 (2016).

43. Maerki, C. et al. Potent and broad-spectrum antimicrobial activity of CXCL14 suggests an immediate role in skin infections. J. Immunol. 182, 507-514 (2009).

44. Hari, P. et al. The innate immune sensor Toll-like receptor 2 controls the senescence-associated secretory phenotype. Sci. Adv. 5, eaaw0254 (2019).

45. $\mathrm{Wu}, \mathrm{C}$. et al. Proteomic analysis reveals IRAK4 as a therapeutic target in chronic lymphocytic leukemia. Blood 130, 3838 (2017).

46. Zhou, J. et al. TAK1 mediates excessive autophagy via p38 and ERK in cisplatin-induced acute kidney injury. J. Cell. Mol. Med. 22, 2908-2921 (2018).

47. Vyrla, D. et al. TPL2 kinase is a crucial signaling factor and mediator of NKT effector cytokine expression in immune-mediated liver injury. J. Immunol. 196, 4298-4310 (2016).

48. $\mathrm{Wu}, \mathrm{X}$. et al. MEK-ERK pathway modulation ameliorates disease phenotypes in a mouse model of Noonan syndrome associated with the Raf1L613V mutation. J. Clin. Invest. 121, 1009-1025 (2011).

49. Mannangatti, P., NarasimhaNaidu, K., Damaj, M. I., Ramamoorthy, S. \& Jayanthi, L. D. A role for p38 mitogen-activated protein kinase-mediated threonine 30-dependent norepinephrine transporter regulation in cocaine sensitization and conditioned place preference. J. Biol. Chem. 290, 10814-10827 (2015).

50. Berger, S. et al. Characterization of GSK'963: a structurally distinct, potent and selective inhibitor of RIP1 kinase. Cell Death Discov. 1, 15009 (2015).

51. Scaffidi, P., Misteli, T. \& Bianchi, M. E. Release of chromatin protein HMGB1 by necrotic cells triggers inflammation. Nature 418, 191-195 (2002).

52. Seki, E. et al. TLR4 enhances TGF- $\beta$ signaling and hepatic fibrosis. Nat. Med. 13, 1324-1332 (2007)

53. Yang, L. \& Seki, E. Toll-like receptors in liver fibrosis: cellular crosstalk and mechanisms. Front. Physiol. 3, 138 (2012).

Publisher's note Springer Nature remains neutral with regard to jurisdictional claims in published maps and institutional affiliations.

(c) The Author(s), under exclusive licence to Springer Nature America, Inc. 2020 


\section{Methods}

Mouse models. Mice were kept in a standard conventional $12 / 12 \mathrm{~h}$ light/dark cycle, $21-24^{\circ} \mathrm{C}, 55 \%$ relative humidity with $12-16$ air changes $\mathrm{h}^{-1}$, and fed commercially available standard chow and water ad libitum. Eight-week-old C57BL6 male mice were injected intraperitoneally with either $500 \mathrm{mg} \mathrm{kg}^{-1}$ body mass APAP in PBS or $300 \mathrm{mg} \mathrm{kg}^{-1}$ of TAA in PBS, $20 \mathrm{~h}$ before sample collection. To avoid known circadian effects on this model, we performed all injections between 13:00 and 14:00. Control mice were injected with vehicle (PBS). For antibiotic treatment, mice were given a cocktail of ampicillin $\left(1 \mathrm{gl}^{-1}\right)$, neomycin $\left(\mathrm{g} \mathrm{g}^{-1}\right)$, vancomycin $\left(0.5 \mathrm{gl}^{-1}\right)$ and metronidazole $\left(1 \mathrm{gl}^{-1}\right)$ in drinking water for 2 weeks. MyD88 and Trif double-KO mice were 8 -week-old males in a C57BL6 background ${ }^{54}$. All experimental procedures involving mice were approved by the local institutional animal care and use committee.

Human samples. All human studies were approved by the Weizmann Institute of Science Bioethics and Embryonic Stem Cell Research oversight committee (Institutional Review Board (IRB) approval no. 699-1) and by the Rabin Medical Center IRB (approval no. RMC-19-0816).

Liver cell isolation. Liver cells were isolated using a modified protocol of Mederacke et al. ${ }^{11}$. In brief, using a peristaltic pump we performed retrograde liver perfusion into the inferior vena cava with three solutions: (1) EGTA $\left(8 \mathrm{gl}^{-1}\right.$ $\mathrm{NaCl}, 0.4 \mathrm{gl}^{-1} \mathrm{KCl}, 88 \mathrm{mgl}^{-1} \mathrm{NaH}_{2} \mathrm{PO}_{4} \cdot \mathrm{H}_{2} \mathrm{O}, 120 \mathrm{mgl}^{-1} \mathrm{Na}_{2} \mathrm{HPO}_{4} \cdot \mathrm{H}_{2} \mathrm{O}, 2.38 \mathrm{gl}^{-1}$ HEPES, $0.35 \mathrm{gl}^{-1} \mathrm{NaHCO}_{3}, 0.19 \mathrm{gl}^{-1}$ EGTA, $0.9 \mathrm{gl}^{-1}$ glucose) for $2 \mathrm{~min}$; (2) pronase $\left(0.4 \mathrm{mg} \mathrm{ml}^{-1}\right.$ protease in EBS buffer: $8 \mathrm{gl}^{-1} \mathrm{NaCl}, 0.4 \mathrm{gl}^{-1} \mathrm{KCl}^{-1} 88 \mathrm{mgl}^{-1}$ $\mathrm{NaH}_{2} \mathrm{PO}_{4} \cdot \mathrm{H}_{2} \mathrm{O}, 120 \mathrm{mgl}^{-1} \mathrm{Na}_{2} \mathrm{HPO}_{4} \cdot \mathrm{H}_{2} \mathrm{O}, 2.38 \mathrm{gl}^{-1} \mathrm{HEPES}, 0.35 \mathrm{gl}^{-1} \mathrm{NaHCO}_{3}$, $\left.0.42 \mathrm{~g} \mathrm{l}^{-1} \mathrm{CaCl}_{2}\right)$ for $5 \mathrm{~min}$; and (3) collagenase $\mathrm{D}\left(0.1 \mathrm{U} \mathrm{ml}^{-1}\right.$ collagenase $\mathrm{D}$ in EBS buffer) for $7 \mathrm{~min}$. The liver was then dissected, placed in cold EBS solution and shaken vigorously with forceps to allow separation of single cells. The solution was filtered through $100-\mu \mathrm{m}$ mesh and hepatocytes were depleted by centrifugation at $30 \mathrm{~g}$ for $5 \mathrm{~min}$. Cells were then collected by centrifugation at $580 \mathrm{~g}$ and resuspended in cold PBS. To enrich for stellate cells, we sorted cells with retinoid fluorescence in the channel excitation 405, emission 450/40 using a BD FACSAria III. We then mixed stellate and unsorted cells, spun them down, resuspended them in PBS with $0.04 \%$ BSA and counted them using a Neubauer chamber, before proceeding to single-cell RNA-seq.

10X library preparation and sequencing. Single cells were captured and processed using the 10X Genomics Chromium 3' Single Cell RNA-seq protocol according to the manufacturer's manual. Subsequently, the libraries were sequenced using NextSeq 500/550 High Output Kit v.2.

Measurement of monocyte infiltration. Mouse livers were finely chopped with sterile scissors and then digested with $4 \mathrm{ml}$ of prewarmed $0.4 \mathrm{mg} \mathrm{ml}^{-1}$ protease and $0.1 \mathrm{U} \mathrm{ml}^{-1}$ collagenase D (EBS buffer; Liver cell isolation) for $30 \mathrm{~min}$ at $37^{\circ} \mathrm{C}$, with shaking. Next, $10 \mathrm{ml}$ of cold PBS was added and the suspension was filtered through $100-\mu \mathrm{m}$ mesh. To deplete hepatocytes, samples were centrifuged at $30 \mathrm{~g}$ for $5 \mathrm{~min}$ and the supernatant was transferred to new tubes. Cells were collected by centrifugation at $580 \mathrm{~g}$. To lyse red blood cells, $1 \mathrm{ml}$ of Gibco ACK Lysing Buffer was added and cells were incubated at room temperature for $1 \mathrm{~min}$. Subsequently, cold PBS was added and cells were collected by centrifugation at $580 \mathrm{~g}$.

Receptors on the cells were first blocked with TruStain FcX anti-mouse CD16/32, then the cells were washed with fluorescent activated cell sorter (FACS) buffer (PBS without calcium and magnesium, 1\% FCS), collected at $580 \mathrm{~g}$ and stained with antibodies CD45-PECy7 (Biolegend, 30F11, no. 103114), F4/80-FitC (Serotec, Cl:A3-1, no. G00018) and Ly6C-APC (Biolegend, HK14, no. 128016) for $1 \mathrm{~h}$ on ice. Finally the cells were washed with FACS buffer, collected at $580 \mathrm{~g}$, resuspended in FACS buffer, filtered through $40-\mu \mathrm{m}$ mesh and measured on a DB LSRFortessa. Data were analyzed using FlowJo software.

Histology. Samples from the left lobe of the liver were fixed in $4 \%$ formaldehyde, embedded in paraffin, sectioned and stained with H\&E. Slides were scored by a blinded veterinary pathologist for necrosis and hemorrhage, on a scale from 0 (healthy) to 5 (most severe).

Inhibitors. The MYC inhibitor KJ-Pyr-9 was injected intraperitoneally $2 \mathrm{~h}$ after injection with $500 \mathrm{mg} \mathrm{kg}^{-1} \mathrm{APAP}$ or corresponding PBS vehicle. Next, $10 \mathrm{mg}$ of KJ-Pyr-9 (Tocris, no. 5306) was dissolved in $1 \mathrm{ml}$ of DMSO and combined with Tween 80 and $5 \%$ dextrose (1:1:8 by volume). Mice were injected with this mixture or corresponding vehicle $(0.5 \mathrm{ml}$ per $20 \mathrm{~g})$ to give a final dose of $25 \mathrm{mg} \mathrm{kg}^{-1}$ body mass.

Inhibitors of MAPK pathway proteins (see table below) were injected intraperitoneally, $1 \mathrm{~h}$ after injection with $500 \mathrm{mg} \mathrm{kg}^{-1} \mathrm{APAP}$ or corresponding PBS vehicle. They were then dissolved in $5 \%$ DMSO in PBS to give a final injection volume of $400 \mu \mathrm{l}$ per $20 \mathrm{~g}$ mouse. Control mice were injected with $5 \%$ DMSO in PBS vehicle.

\begin{tabular}{lll} 
Inhibitor & Target & Dose $\left.\mathbf{( m g ~} \mathbf{~ g g}^{-1}\right)$ \\
\hline PF 06650833 & IRAK4 & 10 \\
\hline 5Z-7-oxozeaenol & MAP3K7 (TAK1) & 10 \\
\hline TC-S 7006 & MAP3K8 (TPL2) & 10 \\
\hline PD0325901 & MEK1/2 (ERK1/2) & 5 \\
\hline SB203580 & P38 & 25 \\
Nec1 & RIP1 & 2
\end{tabular}

Measurement of liver enzyme activity. Measurement of serum ALT and AST level activity was performed initially using a Liver-1 test on an Arkray SPOTCHEM EZ SP-4430, to validate the model (Fig. 1a). All following measurements were done using a Roche Cobas 111 Serum analyzer.

$16 S$ targeted bacterial composition profiling. Colon and small intestine contents were collected postmortem, flash-frozen in liquid nitrogen and stored at $-80^{\circ} \mathrm{C}$. DNA was extracted from the samples with the Invitrogen PureLink Microbiome DNA Purification Kit according to the manufacturer's protocol. The V4 fragment of the 16S rRNA gene was amplified using primers AATGATACGGCGACCACCGAGATCTACACGCTTATGGTAATTGTGTGCCA GCMGCCGCGGTAA and CAAGCAGAAGACGGCATACGAGATAGTCAGCC AGCCXXXXXXXXGGACTACNVGGGTWTCTAAT, where XXXXXXXX denotes a barcode. PCR was performed using TaKaRa Ex Taq DNA Polymerase with $120 \mathrm{ng}$ of genomic DNA input, and $0.4 \mu \mathrm{M}$ of each primer in $25-\mu \mathrm{l}$ reactions. PCR amplification, or its absence in negative controls, was verified by agarose gel electrophoresis. Samples were purified using $0.8 \times$ SPRI beads (Agencourt AMPure $\mathrm{XP})$, and samples were pooled equimolarly and sequenced with a Miseq V2 kit $(2 \times 250$ cycles $)$

Quantification of microbial products in portal vein serum. The following PRR reporter cell lines were obtained from InvivoGen (HEK-Blue TLR and NLR reporter cell lines): TLR2, TLR3, TLR4, TLR5, TLR7, TLR9, NOD1 and NOD2.

Portal vein plasma samples, aseptically collected from mice, were added to reporter cell lines and incubated with HEK-Blue detection medium (Invivogen) according to the manufacturer's instructions.

Immunohistochemistry. Sections $4 \mu \mathrm{m}$ in thickness were deparaffinized, rehydrated, treated for $30 \mathrm{~min}$ with $6 \mathrm{ml}$ of $\mathrm{H}_{2} \mathrm{O}_{2}+200 \mathrm{ml}$ of $70 \%$ methanol $+2 \mathrm{ml}$ of $\mathrm{HCl}$ to block endogenous peroxidase activity, and washed in PBS. Antigen retrieval was done using citric acid. The sections were incubated with blocking solution, processed using an $\mathrm{AB}$ blocking kit and incubated overnight with anticMYC monoclonal antibody (13-2,500, 1:25; Invitrogen). The sections were then incubated with mouse biotin, processed with the $\mathrm{ABC}$ kit and stained with $\mathrm{DAB}$ and hematoxylin. They were then dehydrated, cleared in xylene and coverslipped. Sections were viewed using a microscope under $\times 20$ magnification to monitor the color of the nucleus: a cell was considered positive if the nucleus was stained red/brown. The total numbers of positive and negative nuclei were determined automatically using the 'Image pro' computer program, followed by training the software on manually selected positive and negative cells.

Immunoblotting. Liver tissue was excised and homogenized in RIPA buffer containing protease and phosphatase inhibitors, incubated for $20 \mathrm{~min}$ at $4^{\circ} \mathrm{C}$ and centrifuged for $5 \mathrm{~min}, 2,700 \mathrm{~g}$ at $4^{\circ} \mathrm{C}$. The supernatant was further centrifuged for $30 \mathrm{~min}, 15,000 \mathrm{~g}$ at $4^{\circ} \mathrm{C}$. Samples were run on $12 \%$ acrylamide gels and transferred onto nitrocellulose membranes. Immunoblot analysis was performed using anti-cMYC monoclonal antibody (13-2,500, 1:1,000; Invitrogen), anti-cMyc-Phospho-Ser62 (PA5-104729, 1:1,000; Invitrogen), goat anti-mouse HRP (115-035-205, 1:5,000; Jackson Laboratories) and goat anti-rabbit HRP (111035-003, 1:5,000; Jackson Laboratories). Immunoblot imaging and band intensity quantification were done using the Gel Doc XR+ system (Bio-Rad).

Single-cell RNA-seq data analysis. Mapping. Single-cell RNA-seq data were demultiplexed, mapped to the GRCm38 mouse genome and unique molecular identifiers were counted using the Cell Ranger Single-Cell Software Suite 2.1.1 and bcl2fastq 2.17.1.14

Filtering and doublet removal. First, cells with $<100$ detected transcripts and $>15 \%$ mitochondrial reads were removed. We performed clustering and identified populations of thrombocytes, erythrocytes, neutrophils and mast cells. Next we performed a second filtering using 600 detected transcripts, but did not include the above-mentioned cell populations in this step because these cells have small transcriptomes and they would have been lost. A second step was necessary because there were many low-quality cells with a low threshold. Doublets were then identified by finding clusters of cells expressing gene expression patterns of two cell types simultaneously. The marker sets used were as follows: for stellate cells, Dcn, Colec11, Ecm 1, Cxcl12, Sod3, Angptl6, Rgs5, Reln, Tmem56, Rbp1, G0s2, Rarres2, Acta2 and Tagln; for endothelial cells, Gpihbp1, Aqp1, Clec4g, Dnase1l3, 
Fabp4, Ptprb, Kdr and Gpr182; for hepatocytes, Alb, Ttr, Ambp, Rbp4, Cyp2e1 and Spp1; for T cells, Cd3e, Cd3g, Cd3d, Lat, Thy1, Cxcr6, Nkg7, Cd4, Cd8b1, Klra4, Ncr1, Gzmb, Lck, Txk, Ms4a4b and Ccl5; for B cells, Cd79b, Cd79a, Ms4a1, Siglecg, Fcmr, Cd19 and Fcer2a; for macrophages, dendritic cells and neutrophils, Adgre1, Cd5l, Clec4f, Timd4, Folr2, C1qa, C1qb, C1qc, Vsig4, Xcr1, Cd209a, Itgax, Siglech, Chil3, F13a1, S100a4, Lgals3, Gda, S100a9 and Retnlg; and for erythrocytes, Alas2, $H b b-b t, H b b-b s, H b a-a 2$ and Hba-al.

Clustering. All cells from all 30 samples were first clustered using the R package Seurat v.2.3.4 FindClusters function ${ }^{55}$. Genes present in fewer than three cells were first removed, then highly variable genes were identified as those having a mean of nonzero values $0.0125-3$ and s.d. $>0.5$. Dimensionality reduction was done with principal component analysis, with the first 50 principal components used for clustering.

Because the liver population is very complex, we decided to perform stepwise clustering. We first checked for the expression of Ecm 1, Ptprc, Ptprb, Epcam, $M s l n$ and $A l b$ in the clusters and divided the cells accordingly into seven groups: stellate, immune, endothelial, mesothelial and cycling cells, and cholangiocytes and hepatocytes. Immune cells were classified in the same way and, based on the expression of Agdre1, Cd5l, Ncr1, Cd3e, Cd79b, Retnlg, Cx3cr1 and Stmn1, were split into seven groups: B, T and natural killer cells, neutrophils, Kupffer cells, monocytes and remaining immune cell types. Within these groups, cells were clustered using Seurat FindClusters (Supplementary Figs. 1-12).

Cell type marker identification and annotation. For each cluster, marker genes were identified with the Seurat FindMarkers function and, based on crosschecking of identified markers with known marker genes and comparison to the ImmGen database (available at http://www.immgen.org/), we annotated clusters with cell types. Because many clusters were assigned to macrophages or dendritic cells, we added key marker genes to their cell type description to make comparison to other data easier for the wider audience. Clusters that represented the same cell type were merged - that is, Ly6C-positive monocytes and Cxcr6 T cells.

Functional analysis of cell populations. Gene Ontology analysis and transcription factor binding motif analysis was performed using g:Profiler ${ }^{56}$ with default settings, and multiple hypothesis testing adjustment using all mouse genes as background control. The $\log _{10}$ FDR-adjusted $P$ values were plotted as barplots. For heatmaps, GO lists were obtained from Ensembl BioMart.

Mapping of ligand-receptor interactions. Differentially expressed genes between quiescent stellate, sinusoidal endothelial and Kupffer cells and their corresponding activated counterparts were filtered for ligands from a previously published database ${ }^{30}$ Corresponding receptors were then identified in the database, and both normalized and scaled expression of ligands and receptors were plotted as balloon plots.

Comparison of gene expression between clusters. To compare gene expression between clusters, such as quiescent versus acute stellate cells, we used the Seurat FindMarkers function to define the identity of both clusters ${ }^{55}$.

Comparison of gene expression within a cluster between conditions. To compare gene expression between samples, such as activated stellate cells in GF APAP-induced versus activated stellate cells in SPF APAP-induced mice, we calculated pseudobulk by adding reads from all cells within each cluster in a sample. We then used DESeq2 with default parameters to determine differential expression ${ }^{57}$.

Diffusion maps. Diffusion maps were calculated using the destiny R package ${ }^{38}$. First, Ly6C-positive monocytes were clustered revealing four different subclusters; then, using the Seurat FindMarkers function, we identified the top 50 specific genes for each cluster. Normalized data were filtered for genes specific to these subsets, and these data were used to calculate diffusion maps using Euclidean distances and local-scale parameter sigma, without rotated eigenvalues, and taking the ten nearest neighbors.

$16 \mathrm{~S}$ V4 amplicon sequence analysis. $16 \mathrm{~S}$ amplicon sequences were analyzed using Qiime2 (ref. ${ }^{58}$ ), and sequencing reads were demultiplexed with demux plug-in. Thirty-one poor-quality bases were trimmed from the reverse read, and one base from forward read, combined, denoised and amplicon sequence variants (ASVs) was called with dada2. Sequences were aligned using Mafft, masked and a phylogenetic tree constructed using phylogeny fasttree; reads were then rarefied to 20,000 reads per sample. Taxonomic assignment to ASVs was done using feature-classifier classify-sklearn and Greengenes $13 \_8,99 \%$ operational taxonomic units. Differential abundance analysis was done with a two-sided Wilcoxon test and Benjamini-Hochberg FDR correction.

Data integrity check. Figures, supplementary figures and supplementary information panels were checked for data integrity using the Proofig pipeline.
Reporting Summary. Further information on research design is available in the Nature Research Reporting Summary linked to this article.

\section{Data availability}

The small cytoplasmic RNA-seq data have been deposited with ArrayExpress under accession no. E-MTAB-8263, and 16S sequencing data with the European Nucleotide Archive under accession no. ERP116956. Source data are provided with this paper.

\section{References}

54. Yamamoto, M. Role of adaptor TRIF in the MyD88-independent Toll-like receptor signaling pathway. Science 301, 640-643 (2003).

55. Stuart, T. et al. Comprehensive integration of single-cell data. Cell 177, 1888-1902 (2019).

56. Reimand J. et al. g:Profiler-a web-based toolset for functional profiling of gene lists from large-scale experiments. Nucleic Acids Res. 35, 193-200 (2007).

57. Love, M. I., Huber, W. \& Anders, S. Moderated estimation of fold change and dispersion for RNA-seq data with DESeq2. Genome Biol. 15, 550 (2014).

58. Bolyen, E. et al. QIIME 2: reproducible, interactive, scalable, and extensible microbiome data science. Nat. Biotechnol. 37, 852-857 (2019).

\section{Acknowledgements}

We thank members of the Elinav laboratory and the DKFZ cancer-microbiome division for discussions. We thank C. Bar-Nathan for dedicated assistance with animal work. A.A.K. is a recipient of EMBO Long Term Fellowship no. 2016-1088 and the European Union's Horizon 2020 research and innovation program under Marie Sklodowska-Curie grant agreement no. 747114. I.A. is supported by the Chan Zuckerberg Initiative, an HHMI international scholar award, a European Research Council consolidator grant (ERC-COG, no. 724471-Hem-Tree2.0), the Thompson Family Foundation, an MRA established investigator award (no. 509044), the Israel Science Foundation (no. 703/15), the Ernest and Bonnie Beutler Research Program for Excellence in Genomic Medicine, a Helen and Martin Kimmel award for innovative investigation, a NeuroMac DFG/ Transregional Collaborative Research Center grant, International Progressive MS Alliance/NMSS (no. PA-1604-08459), an Adelis Foundation grant and the SCA award of the Wolfson Foundation. E.E. is supported by Yael and Rami Ungar; the Leona M. and Harry B. Helmsley Charitable Trust; the Adelis Foundation; the Pearl Welinsky Merlo Scientific Progress Research Fund; the Lawrence and Sandra Post Family Foundation; the Daniel Morris Trust; the Park Avenue Charitable Fund; the Hanna and Dr Ludwik Wallach Cancer Research Fund; the Howard and Nancy Marks Charitable Fund; Aliza Moussaieff; the estates of Malka Moskowitz, Myron H. Ackerman and Bernard Bishin (for the WIS-Clalit Program); Donald and Susan Schwarz; the V. R. Schwartz Research Fellow Chair; grants funded by the European Research Council; Israel Science Foundation; Israel Ministry of Science and Technology; Israel Ministry of Health; the Helmholtz Foundation; the Else Kroener Fresenius Foundation; the Garvan Institute; the European Crohn's and Colitis Organization; Deutsch-Israelische Projektkooperation; and Welcome Trust. E.E. is the incumbent of the Sir Marc and Lady Tania Feldmann Professorial Chair; a senior fellow, Canadian Institute of Advanced Research and an international scholar; and The Bill \& Melinda Gates Foundation and Howard Hughes Medical Institute.

\section{Author contributions}

A.A.K. and E.E. designed, analyzed and interpreted all experiments, and wrote the manuscript. A.A.K performed all experiments with the help of S.F., N.Z., G.M., S.H., A.L., M.D.-B. and H.S. D.R. and E.Z. provided experimental support. T.M.S. assisted with cell sorting. A.H. assessed tissue histology. A.T. and A.S. provided human clinical data, insights and material. I.A. and E.E. supervised the study.

\section{Competing interests}

E.E. is a paid consultant at DayTwo and BiomX. None of this work is related to, funded or endorsed by, shared or discussed with or licensed to any commercial entity.

\section{Additional information}

Extended data is available for this paper at https://doi.org/10.1038/s41591-020-1102-2. Supplementary information is available for this paper at https://doi.org/10.1038/ s41591-020-1102-2.

Correspondence and requests for materials should be addressed to I.A. or E.E. Reprints and permissions information is available at www.nature.com/reprints. Editor recognition statement Joao Monteiro was the primary editor on this article and managed its editorial process and peer review in collaboration with the rest of the editorial team. 
A

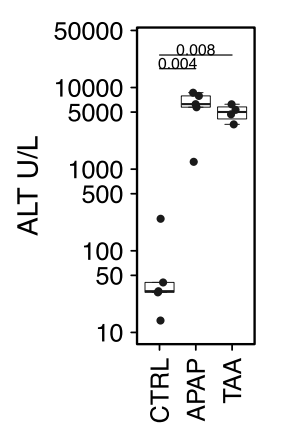

C

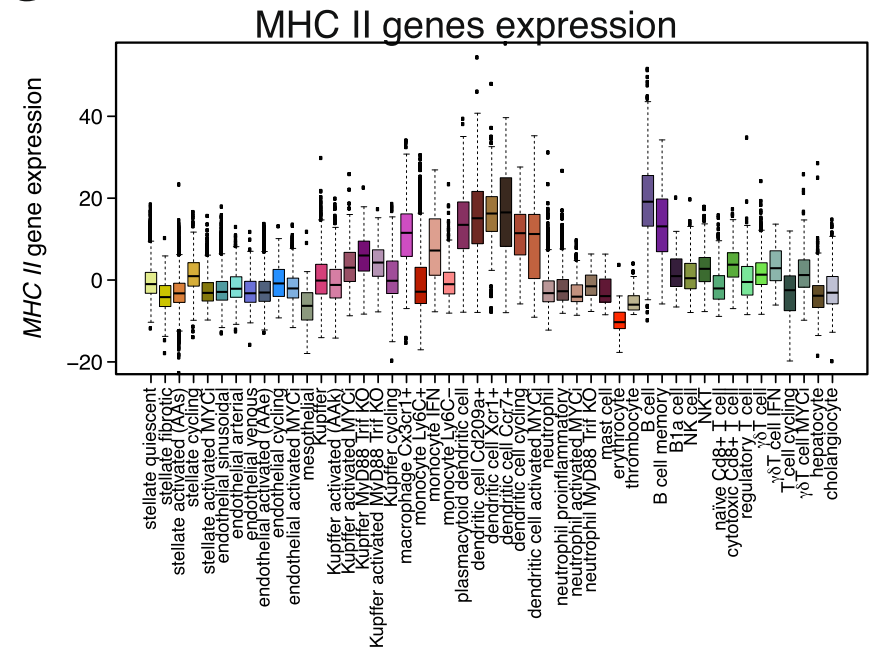

$E$

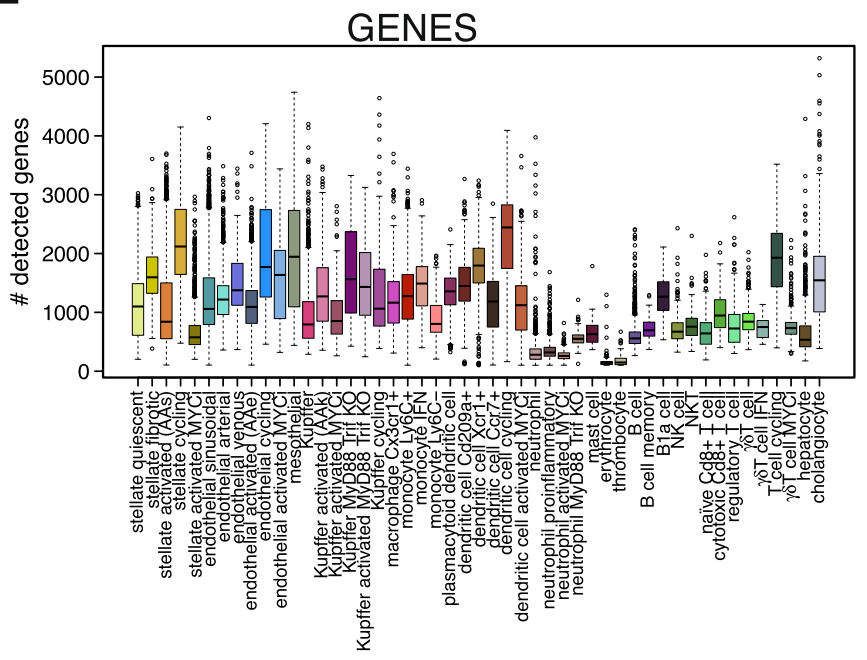

B

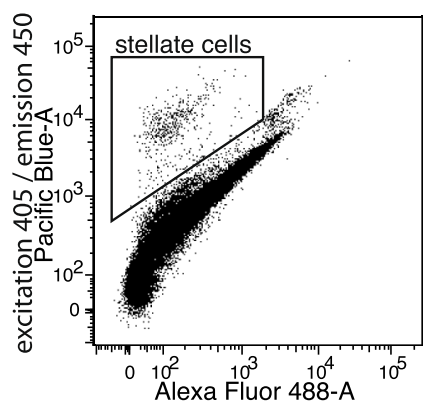

D

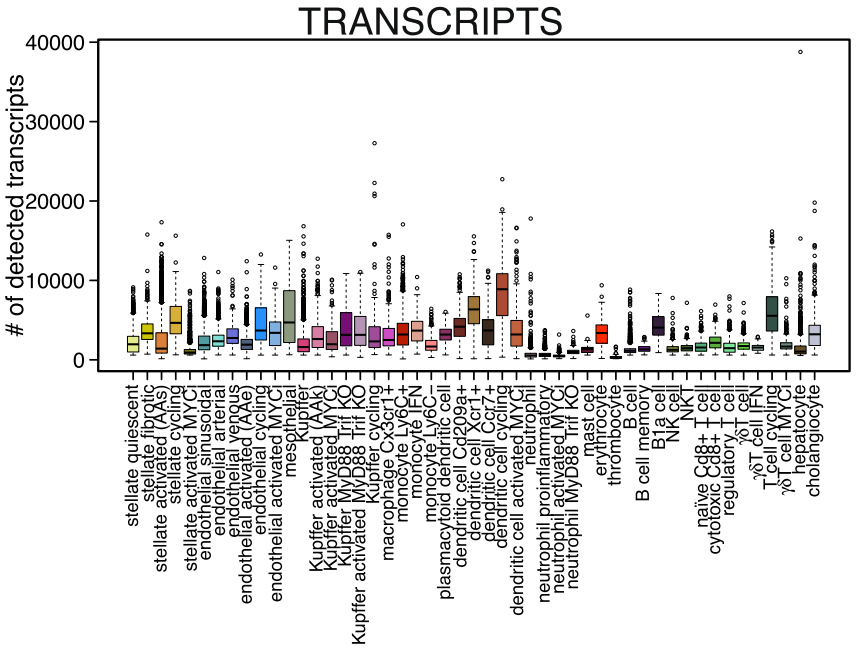

Extended Data Fig. 1 | See next page for caption. 
Extended Data Fig. 1 | Acute liver failure model and characterisation of cell type. a, Activity of hepatic enzymes aspartate transaminase (AST) and alanine transaminase (ALT) in serum of mice injected with APAP and TAA, significance was determined using one-sided Wilcoxon test; $n=5$ for each group. Boxplot shows $25^{\text {th }}$ to $75^{\text {th }}$ percentiles with $50^{\text {th }}$ denoted with a line, whiskers show 1.5 times interquartile range or maximum or minimum if they are smaller than that. b, FACS of retinoid fluorescence positive cells. c, Boxplot showing sum of normalised and scaled expression of $\mathrm{MHCll}$ in each cell type stellate quiescent $n=7282$, stellate fibrotic $n=140$, stellate activated (AAs) $n=6470$, stellate cycling $n=180$, stellate activated MYCi $n=2013$, endothelial sinusoidal $n=5736$, endothelial arterial $n=932$, endothelial venous $n=167$, endothelial activated (AAe) $n=4928$, endothelial cycling $n=54$, endothelial activated MYCi $n=2287$, mesothelial $n=128$, Kupffer $n=2696$, Kupffer activated (AAk) $n=697$, Kupffer activated MYCi $n=384$, Kupffer MyD88 Trif KO $n=265$, Kupffer activated MyD88 Trif KO $n=406$, Kupffer cycling $n=155$, macrophage Cx3cr1 $n=280$, monocyte Ly6C $n=5002$, monocyte IFN $n=93$, monocyte Ly6C $n=293$, plasmacytoid dendritic cell $n=295$, dendritic cell Cd209a+ $n=542$, dendritic cell Xcr ${ }^{+} n=302$, dendritic cell Ccr7 ${ }^{+} n=119$, dendritic cell cycling $n=83$, dendritic cell activated MYCi $n=297$, neutrophil $n=3771$, neutrophil proinflammatory $n=1804$, neutrophil activated MYCi $n=582$, neutrophil MyD88 Trif KO $n=152$, mast cell $n=41$, erythrocyte $n=114$, thrombocyte $n=97, B$ cell $n=2063$, B cell memory $n=142$, B1a cell $n=21, N K$ cell $n=381, N K T n=344$, naïve Cd8 ${ }^{+}$T cell $n=897$, cytotoxic Cd8 ${ }^{+} T$ cell $n=174$, regulatory T cell $n=408, y \delta T$ cell $n=1373$,

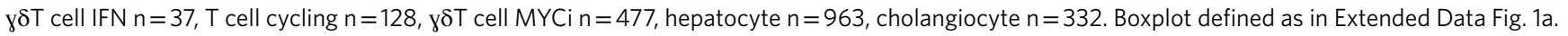
d, Boxplot showing number of transcripts expressed in each cell type, number of cells as in c. Boxplot defined as in Extended Data Fig. 1a. e, Boxplot showing number of genes detected in each cell type, which corresponds to the number of detected unique transcripts, number of cells as in c. Boxplot defined as in Extended Data Fig. 1a. 

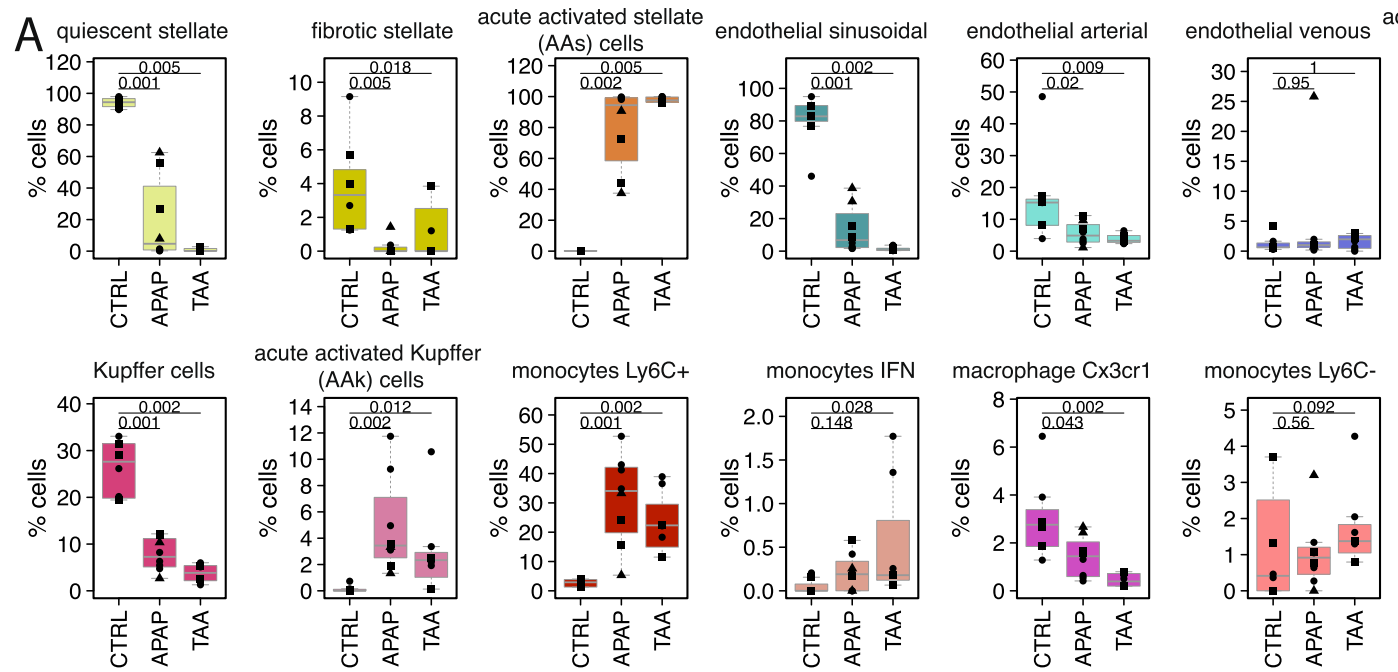

acute activated endothelial

monocytes Ly6C+

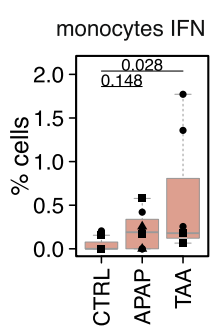

macrophage $\mathrm{C} \times 3 \mathrm{cr} 1$
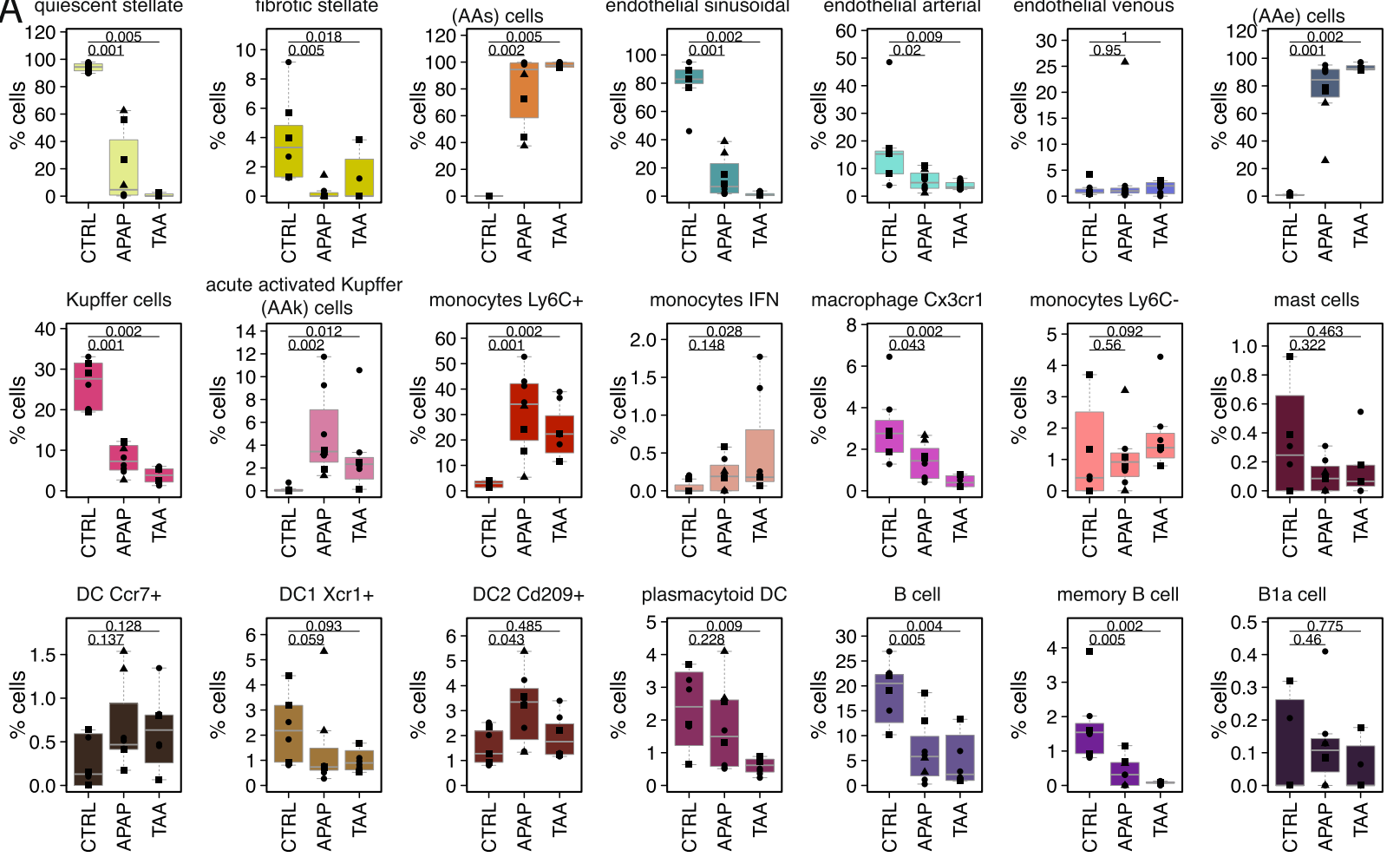

NKT cell
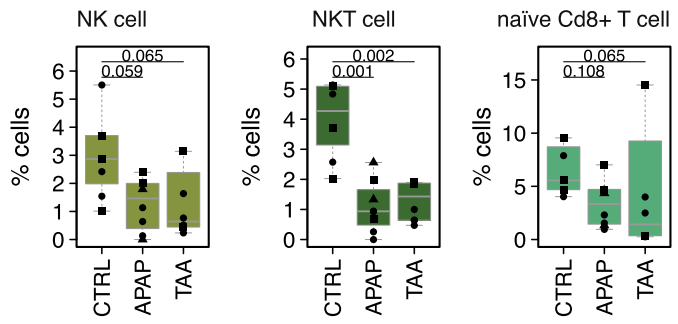

cytotoxic $\mathrm{Cd} 8+\mathrm{T}$ cell
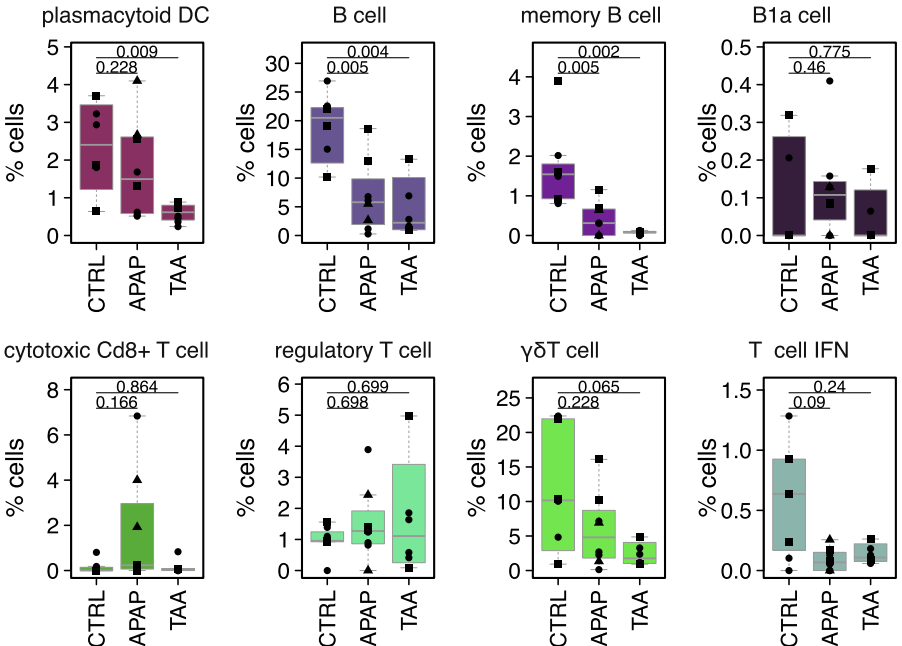

$\mathrm{T}$ cell IFN

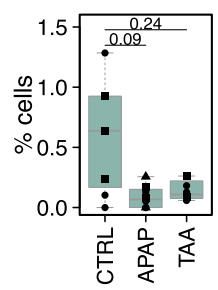

neutrophils
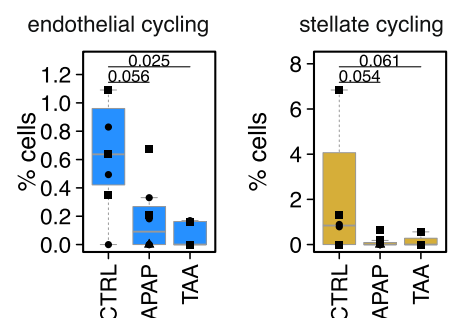

proinflammatory
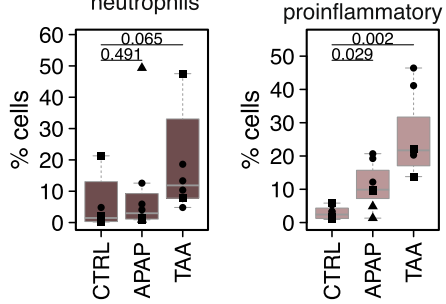

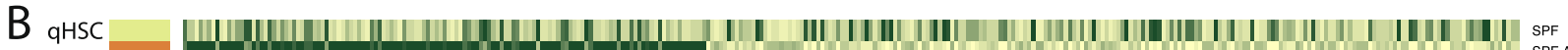
AAs s SPFAA

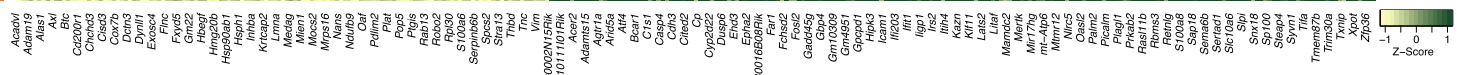

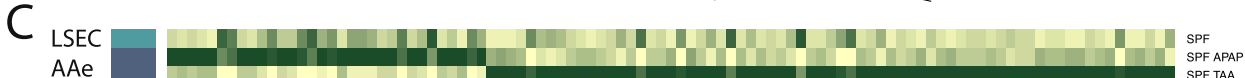

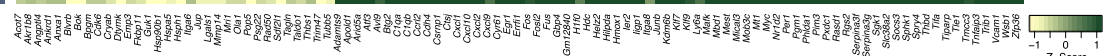

D

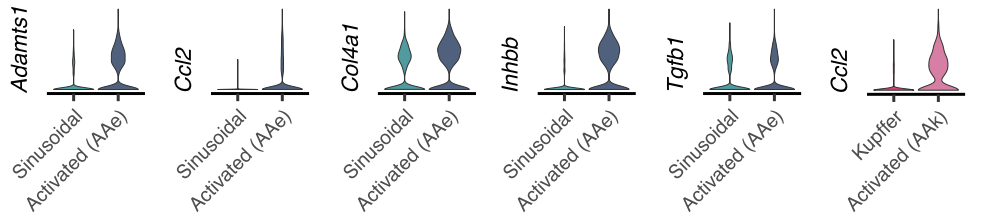

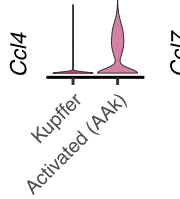

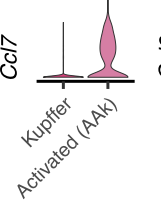

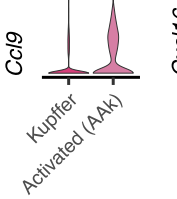

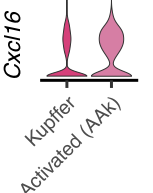

Extended Data Fig. 2 | See next page for caption. 
Extended Data Fig. 2 | Cell abundance changes in acute liver failure and differences between APAP and TAA models. a, Percentage of cell populations in control mice $(n=6), \operatorname{APAP}(n=8)$ and TAA $(n=6)$ treated mice, significance was determined using two-sided Wilcoxon test. Boxplot defined as in Extended Data Fig. 1a. Data points from SPF samples denoted as $\mathbf{0}, \mathrm{GF}-\mathbf{\square}$ and ABX - $\mathbf{\Delta} \mathbf{b}$, Heatmap showing differentially expressed genes in AAs between APAP and TAA treated mice. $\mathbf{c}$, Heatmap showing differentially expressed genes in AAe between APAP and TAA treated mice. e, Violin plots showing normalised and scaled expression of example chemokines upregulated in activated Kupffer cells. $\mathbf{d}$, Violin plots showing normalised and scaled expression of example chemokines, cytokines and extracellular matrix modifiers upregulated in activated endothelial cells. e, Violin plots showing normalised and scaled expression of example chemokines upregulated in activated Kupffer cells. 

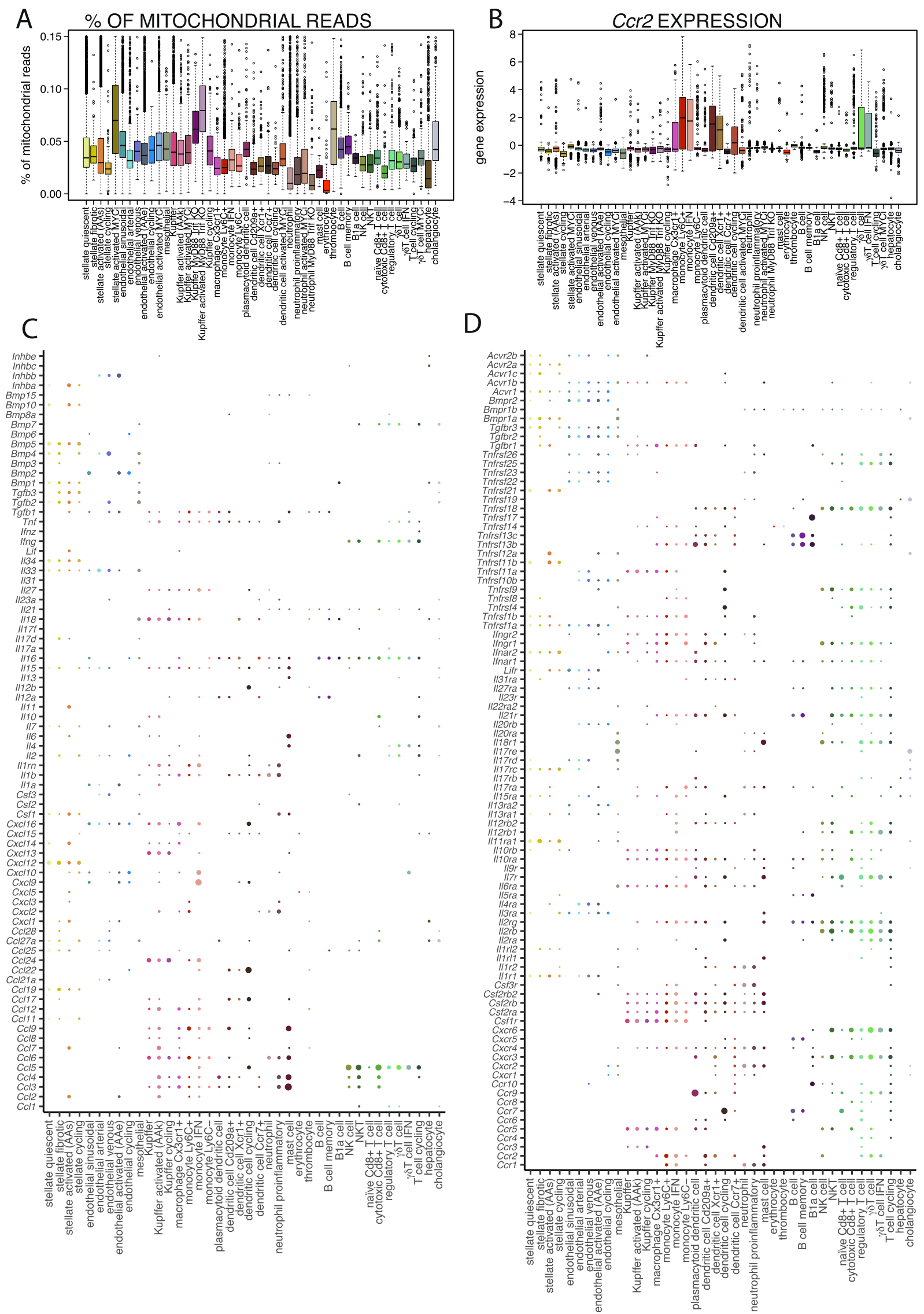

Extended Data Fig. 3 | Receptors and ligands in ALF. a, Boxplot showing percent of reads mapping to the mitochondrial genome in each cell type, number of cells as in Extended Data Fig. 1c. b. Boxplot showing normalised and scaled expression of Ccl2 receptor, Ccr 2 in all cell types, number of cells as in Extended Data Fig. 1c. c, d, Baloon plots showing expression of (c) chemokines and cytokines and (d) their receptors. 
A

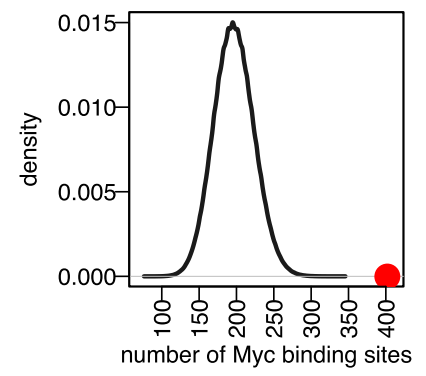

B

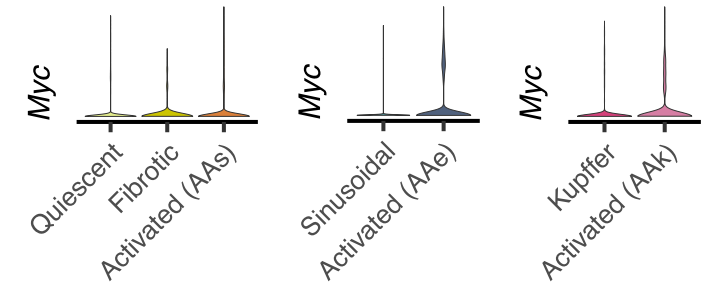

D
C

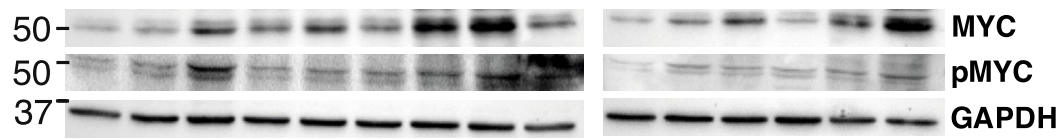

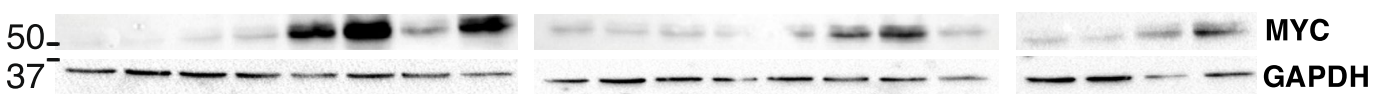
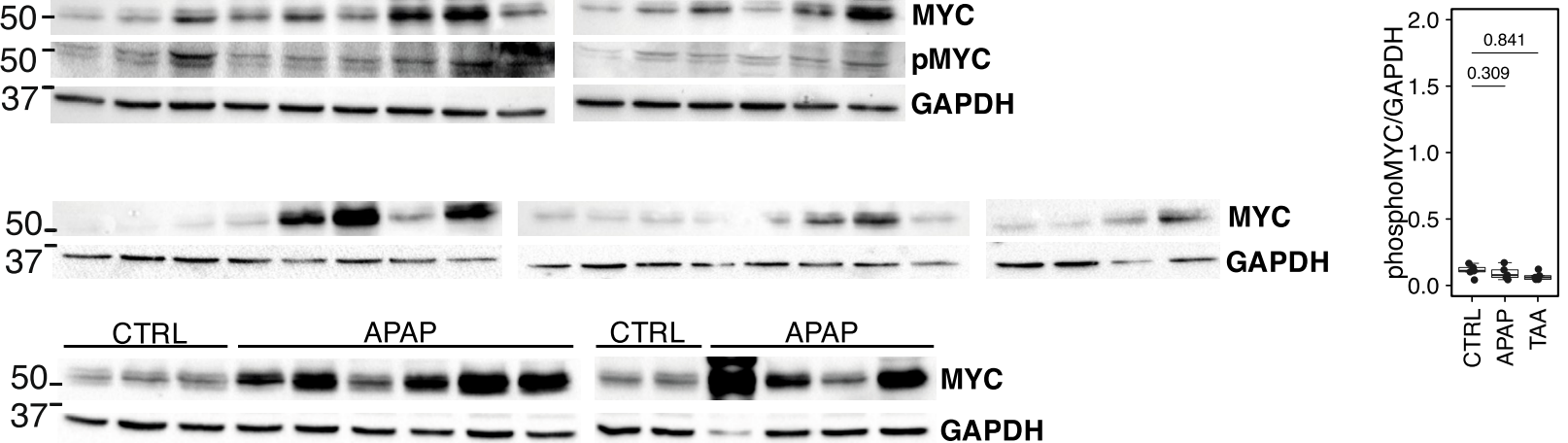

$E$
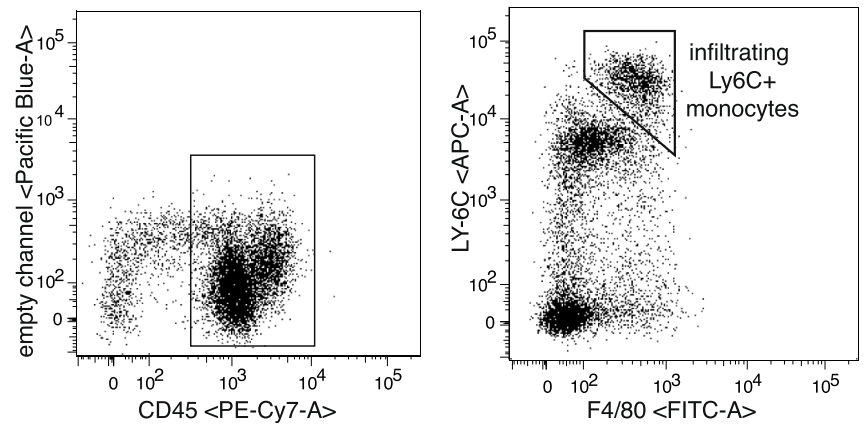

$\mathrm{F}$

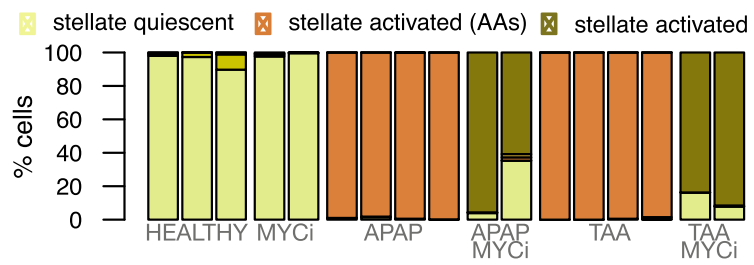

endothelial sinusoidal endothelial arterial endothelial activated (AAe) $\mathrm{X}$ endothelial activated MYCi

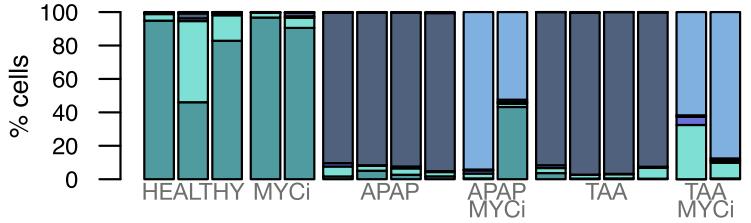

Kupffer Kupffer activated (AAk) Kupffer activated $\mathrm{MYCi}$ $\triangle$ monocyte Ly6C+ $\triangle$ neutrophil $\triangle$ neutrophil proinflammatory neutrophil activated MYCi

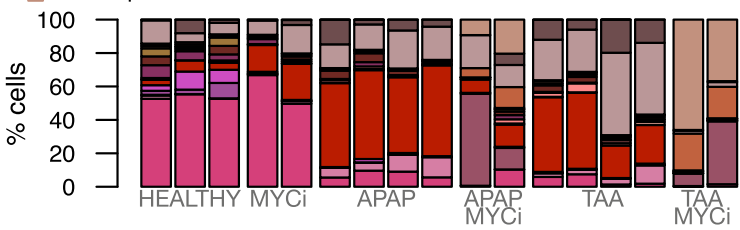

NK cell $\triangle \mathrm{NKT} \otimes$ naïve $\mathrm{Cd} 8+\mathrm{T}$ cell $\mathrm{T}$ regulatory $\mathrm{T}$ cell $\gamma \delta \mathrm{T}$ cell $\mathrm{T}$ cell cycling $\boldsymbol{\nabla} \delta \mathrm{T}$ cell $\mathrm{MYCi}$

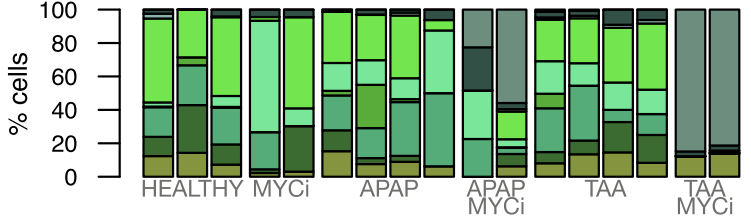


Extended Data Fig. 4 | Cellular states upon MYC inhibition. a, Density plot of permutation analysis of number of MYC binding sites in randomly chosen 77 genes (black) in comparison to 77-gene signature (red) b Violin plots showing mild upregulation of expression of Myc in activated cells. c, Western blots of MYC and phospho-MYC and $\mathbf{d}$ quantification of phospho-MYC Western blot of control mice $(n=5), \operatorname{APAP}(n=5)$ and TAA $(n=5)$ treated mice. Boxplot defined as in Extended Data Fig. 1a. Significance was determined using two-sided Wilcoxon test. e, FACS gating strategy to identify Ly6C-positive monocytes. $\mathbf{f}$, Barplot showing relative frequencies of cells in healthy and mice with ALF in the presence and absence of MYCi. 

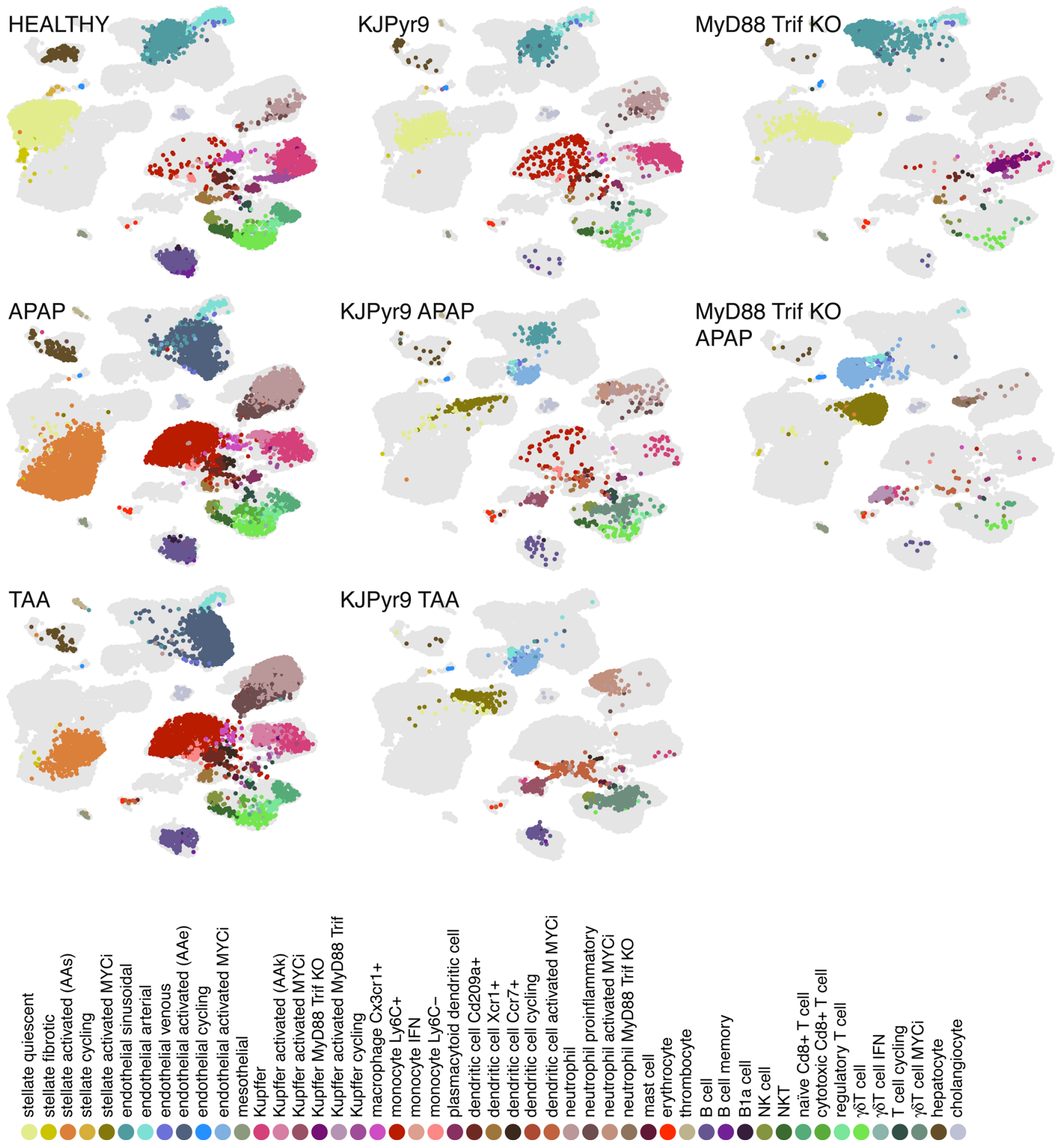

Extended Data Fig. 5 | Cellular states upon MYC inhibition. UMAP showing distribution of cell clusters in healthy, APAP and TAA treated mice in the presence and absence of MYCi. 
A

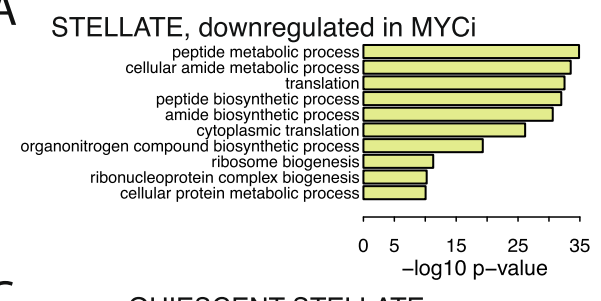

C

QUIESCENT STELLATE
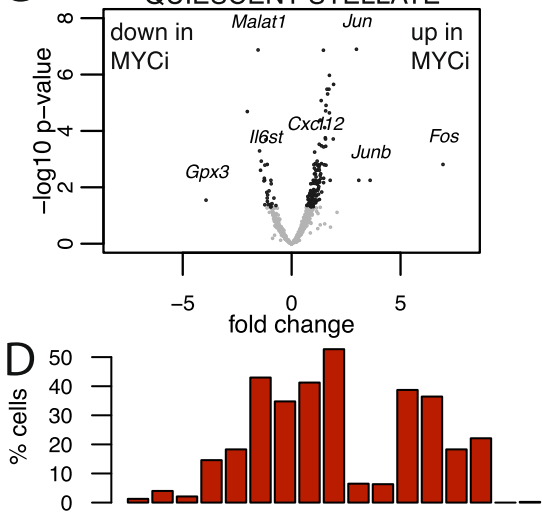

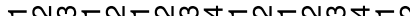

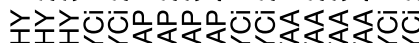

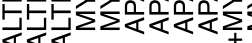
这我 யயய

这部

G

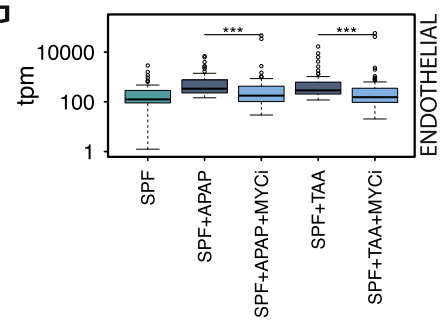

J STELLATE, downregulated in AAs (vs MYCi)

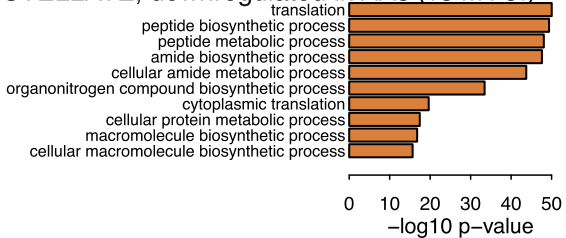

KUPFFER, downregulated in AAK (vs MYCi)

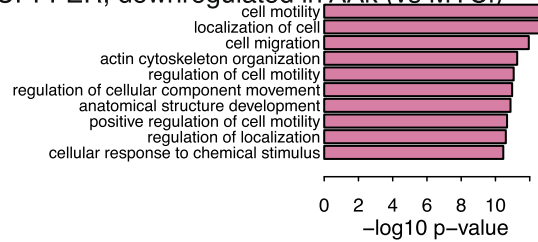

ENDOTHELIAL, downregulated in AAe (vs MYCi)

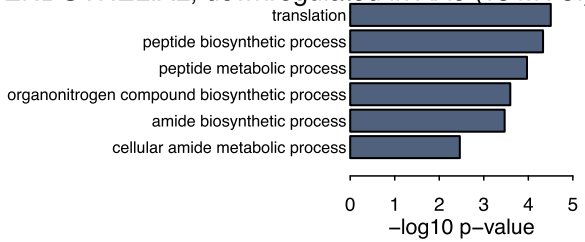

B

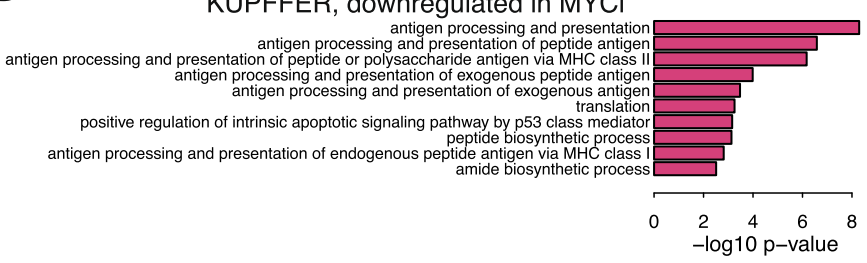

SINUSOIDAL ENDOTHELIAL

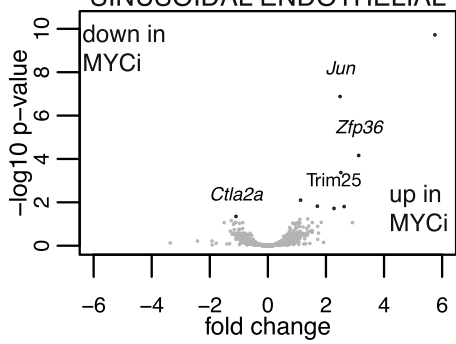

$\left.\begin{array}{rr}\mathrm{E} & 60 \\ \frac{0}{50} & 40 \\ \overline{\mathrm{E}} & 40 \\ \circ & 30 \\ \circ & 20 \\ 0 & 10 \\ & 0\end{array}\right]$

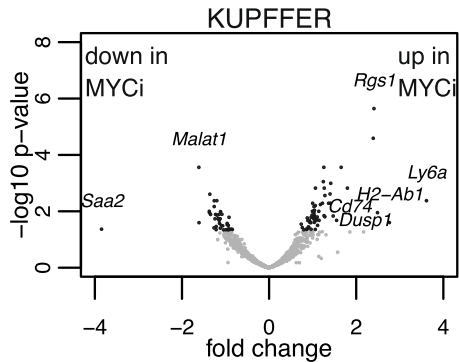

$\mathrm{F}$
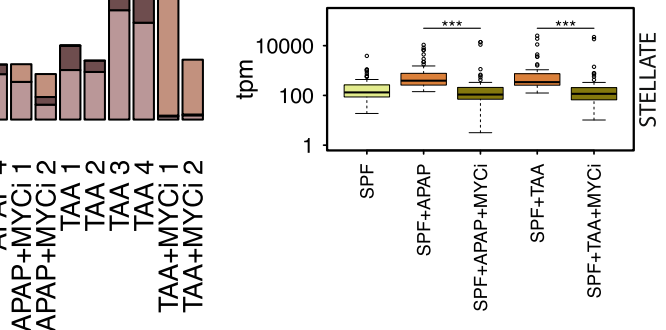

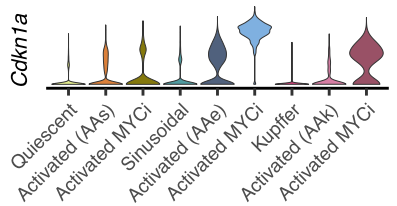

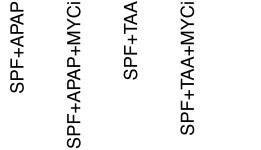

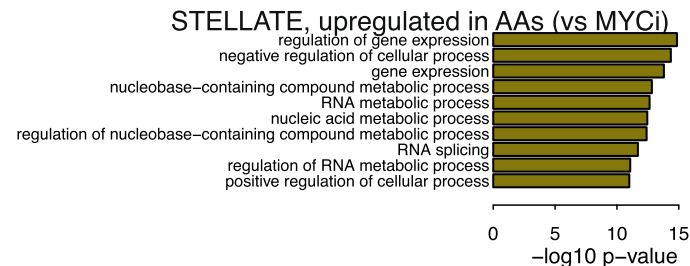

KUPFFER, upregulated in AAk (vs MYCi)

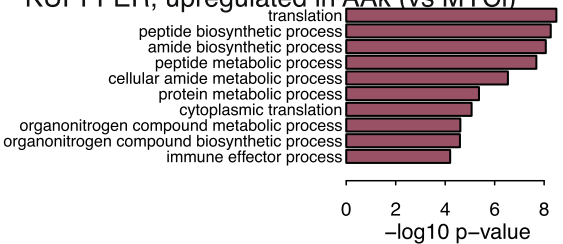

ENDOTHELIAL, upregulated in AAe (vs MYCi)

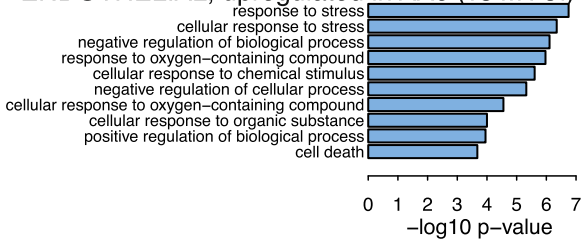

Extended Data Fig. 6 | See next page for caption. 
Extended Data Fig. 6 | Effect of MYC inhibition on gene expression. a, b, Gene ontology term enrichment analysis of genes differentially expressed in healthy mice and healthy mice treated with MYCi in stellate cells and in Kupffer cells. GO analysis was performed with GProfiler using standard settings, p-values shown are corrected for multiple hypothesis testing using g:SCS algorithm. c, Volcanoplots showing differentially abundant genes healthy mice and healthy mice treated with MYCi. Y-axis value depicts multiple hypothesis testing corrected $p$-value calculated using DESeq2 package. d, e, Barplot showing infiltration of (d) Ly6C-positive monocytes and (e) neutrophils in the presence and absence of MYCi. Different colors of bars denote subpopulations of neutrophils; legend as in Extended Data Fig. 5. f-h, Boxplots showing expression of 77-gene signature in healthy mice, mice treated with APAP or TAA and mice treated with APAP and MYCi SPF $(n=3, c S=1999, c E=1463, c K=659), \operatorname{SPF}+\operatorname{APAP}(n=4, c S=4339, c E=1517, c K=265)$, $S P F+T A A(n=4, c S=910, c E=1456, c K=285)$, in presence of MYCi: $S P F+A P A P+M Y C i(n=2, c S=251, c E=303, c K=125)$ and $S P F+T A A+M Y C i$ $(n=2, c S=198, c E=512, c K=233)$, ${ }^{\star \star \star}$ denotes $p$-value $<0.001, n=$ number of mice, $c S=$ number of stellate cells, $c E=$ number of endothelial cells, $c K$ $=$ number of Kupffer cells. Boxplot defined as in Extended Data Fig. 1a. Significance was determined using one-sided Wilcoxon test. $p$-values in stellate cells: SPF + APAP vs SPF + APAP + MYCi 1.135.10-15, SPF + TAA vs SPF + TAA + MYCi 2.662.10-14; in endothelial cells: SPF + APAP vs SPF + APAP + MYCi $5.196 \cdot 10^{-6}$, SPF + TAA vs SPF + TAA + MYCi 4.935·10-6; in Kupffer cells: SPF + APAP vs SPF + APAP + MYCi 1.287.10-8, SPF + TAA vs SPF + TAA + MYCi $1.517 \cdot 10^{-7}$ (i) Violin plot showing normalised and scaled expression of Cdkn1a in three activated cells types. $\mathbf{j}$, Gene ontology term enrichment analysis of genes differentially expressed in APAP and TAA treated mice with and without MYC inhibitor in stellate, endothelial and Kupffer cells. GO analysis was done as in Extended Data Fig. 6a-b. 

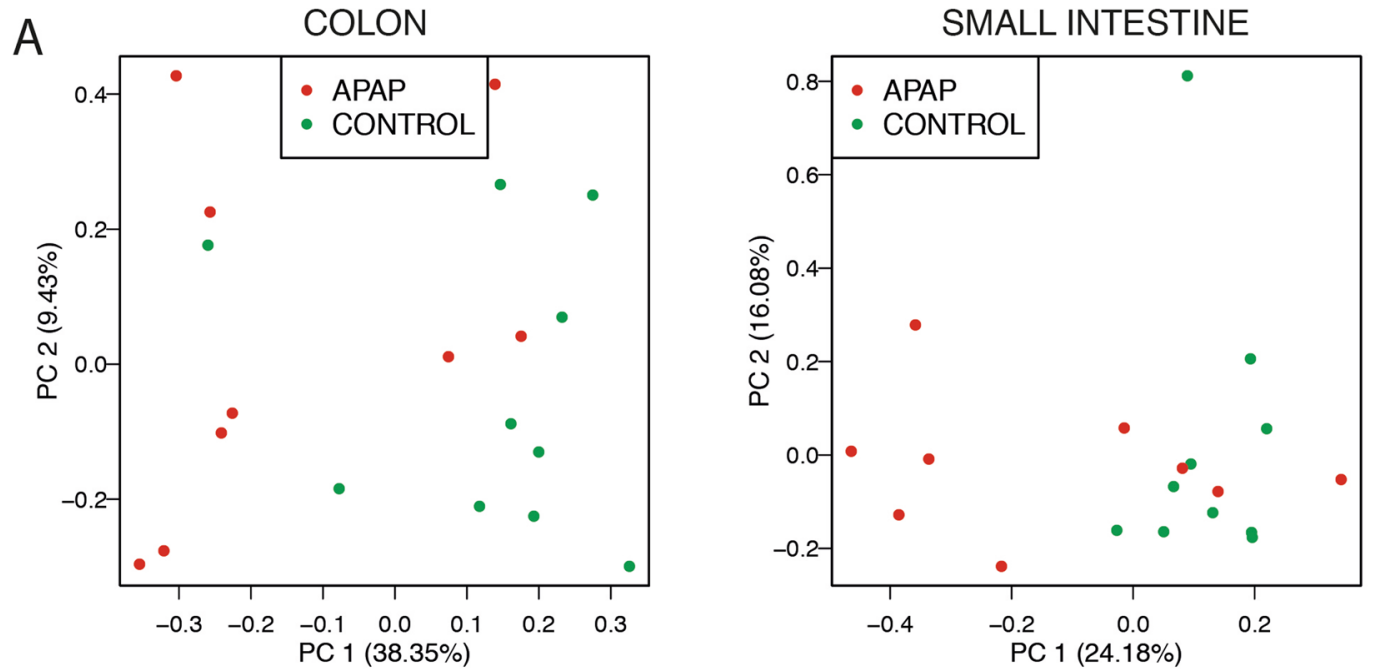

B

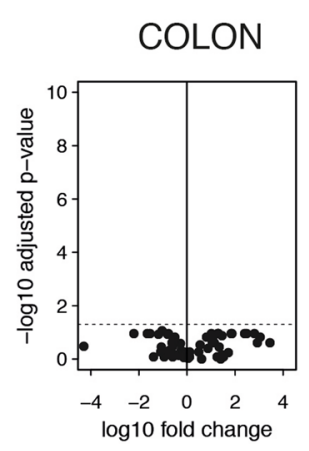

SMALL INTESTINE

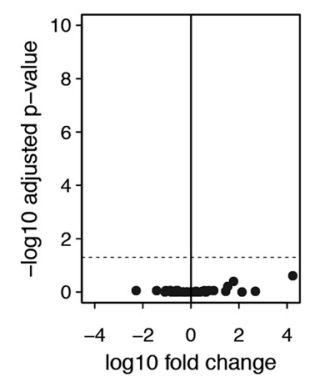

C

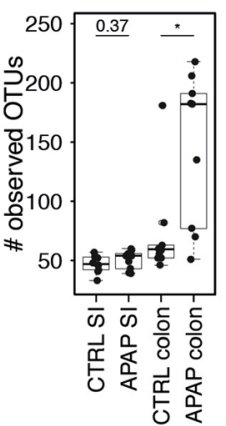

ALPHA DIVERSITY METRICS

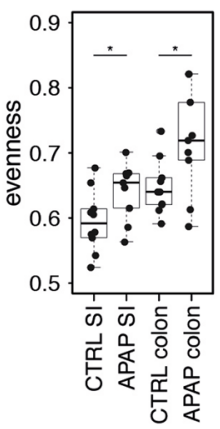

D
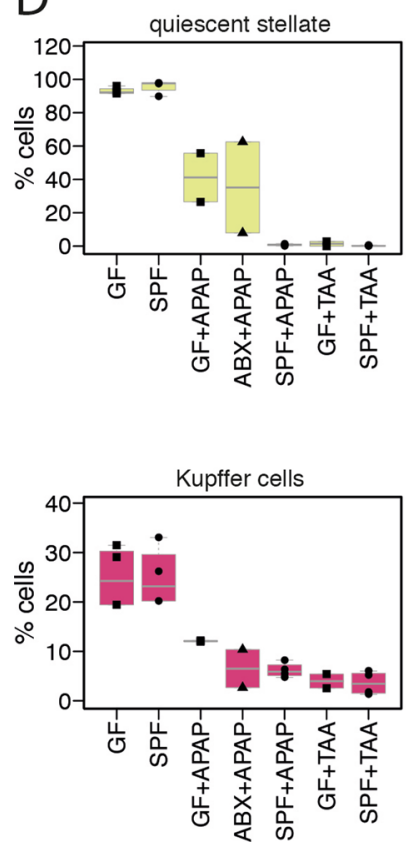

acute activated stellate

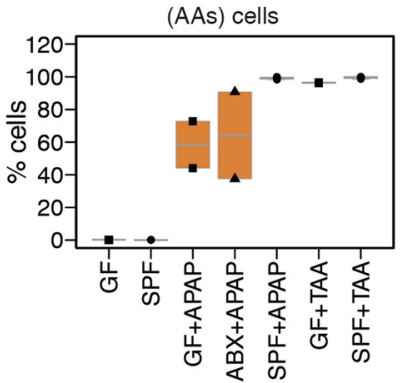

acute activated Kupffer

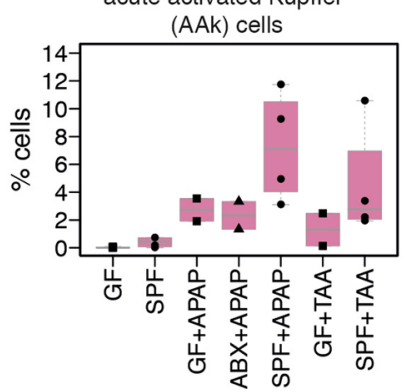

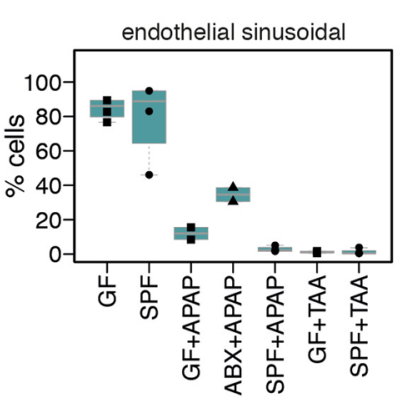
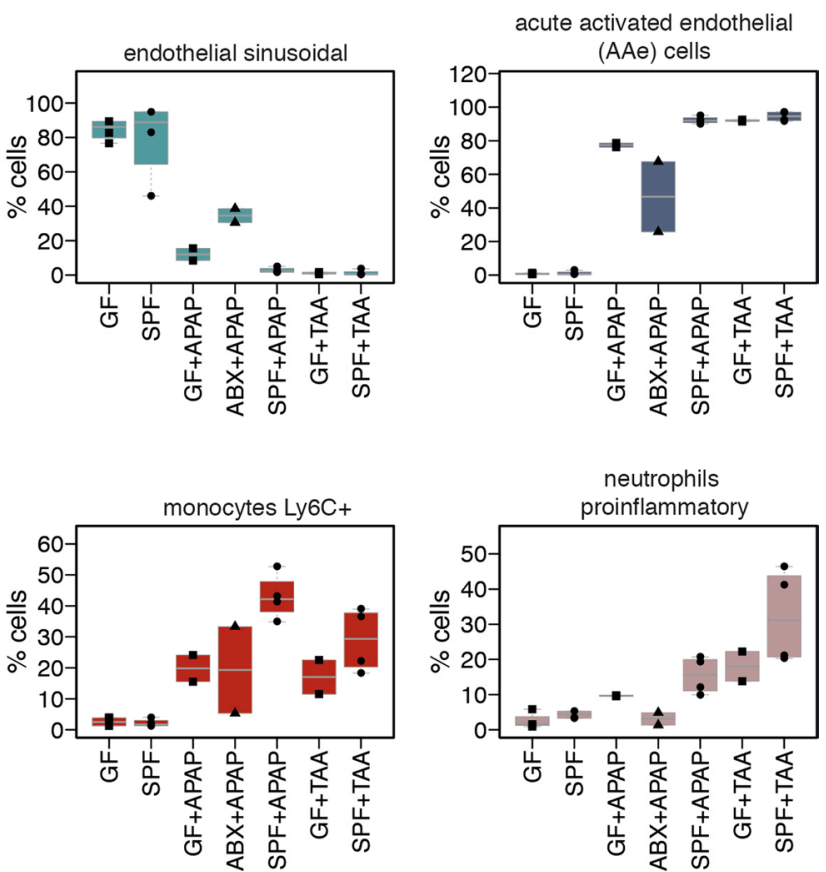

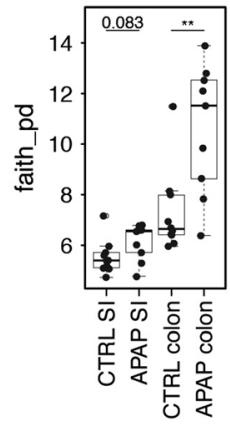

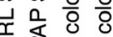

点这

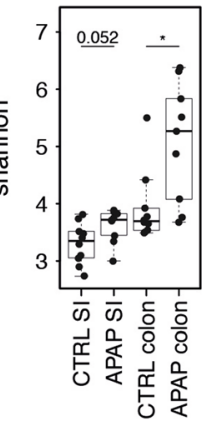

Extended Data Fig. 7 | See next page for caption. 
Extended Data Fig. 7 | Microbiome in acute liver failure. a, PCA of $16 \mathrm{~S}$ microbiome ASV abundance data in the small intestine and in the colon of mice treated with APAP (intraperitoneal injection) and PBS control. b. Volcanoplots showing differential abundance analysis fold change and Benjamini-Hochberg adjusted $p$-values obtained with two-sided Wilcoxon test $\mathbf{c}$, Alpha diversity metrics in control mice (colon $n=10$, small intestine $n=10$ ) and APAP treated mice (colon $n=9$, small intestine $n=9$ ), significance was determined using two-sided t-test. P-value for comparison between control and APAP treated mice for OTUs in the small intestine 0.3706 , colon 0.0103 , for evenness in the small intestine 0.04177 , colon 0.04094 , for Shannon diversity index in the small intestine 0.05235 , colon 0.01271 , for Faith's phylogenetic diversity index in the small intestine 0.08351 , colon 0.00511 . Boxplot defined as in Extended Data Fig. 1a, ${ }^{\star \star}$ denotes $p$-value $<0.01$, ${ }^{\star}$ denotes $p$-value $<0.05$. d, Box plots showing percent of cells, quiescent stellate cells and AAs within stellate cell populations, endothelial sinusoidal cells and AAe within endothelial cells and the Kupffer cells, AAk cells, monocytes and neutrophils within immune cells. GF $n=2, S P F n=3, G F+A P A P n=2, A B X+A P A P n=2, S P F+A P A P n=4, G F+T A A n=2, S P F+T A A n=4$. Boxplot defined as in Extended Data Fig. 1a. 
A

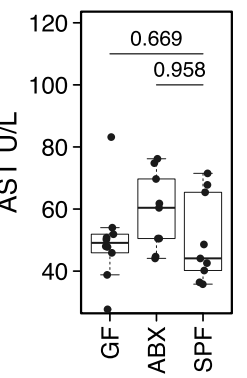

B

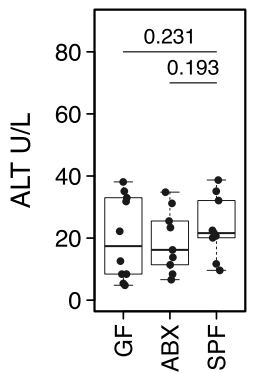

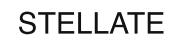

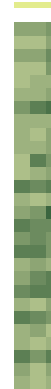

C

ENDOTHELIAL
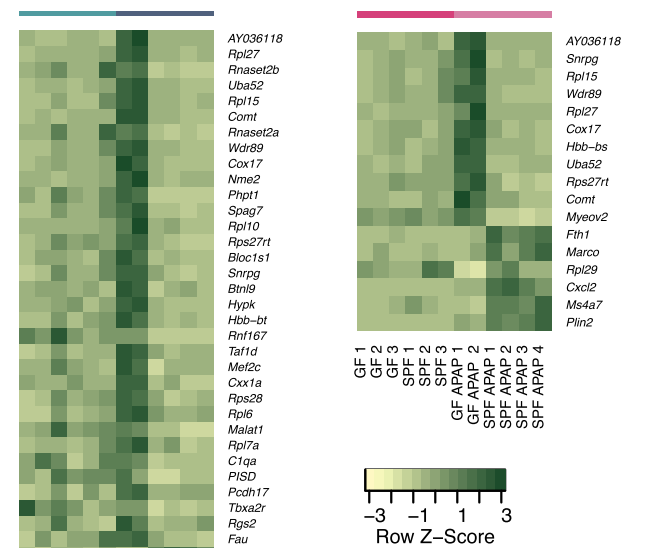

D
E

MONOCYTES

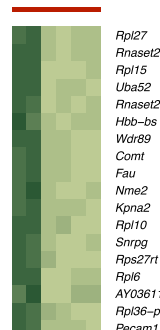

Rpost-pss
Pocamt

Blocts1
Ubxn11

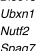

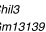
Phptr

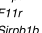

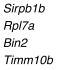
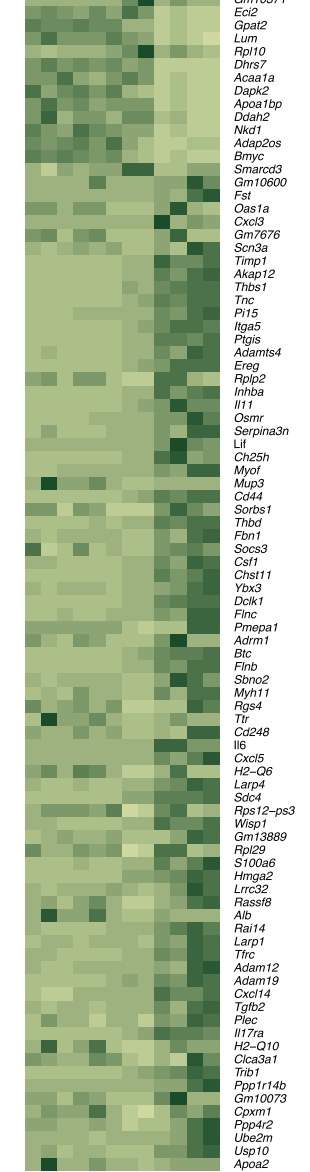

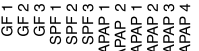

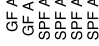

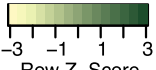

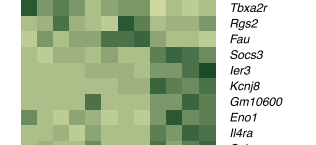
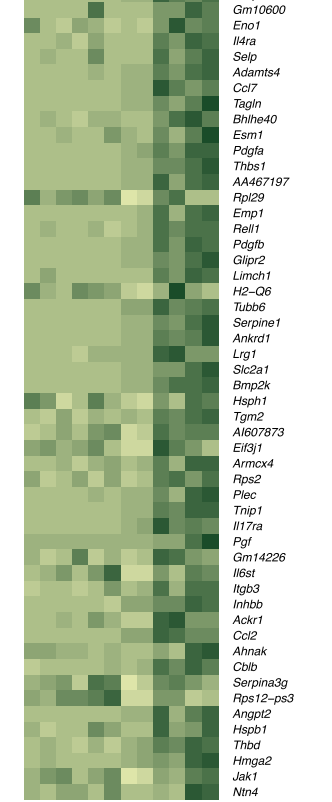

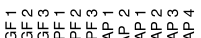

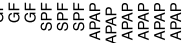

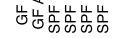

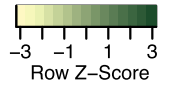


A

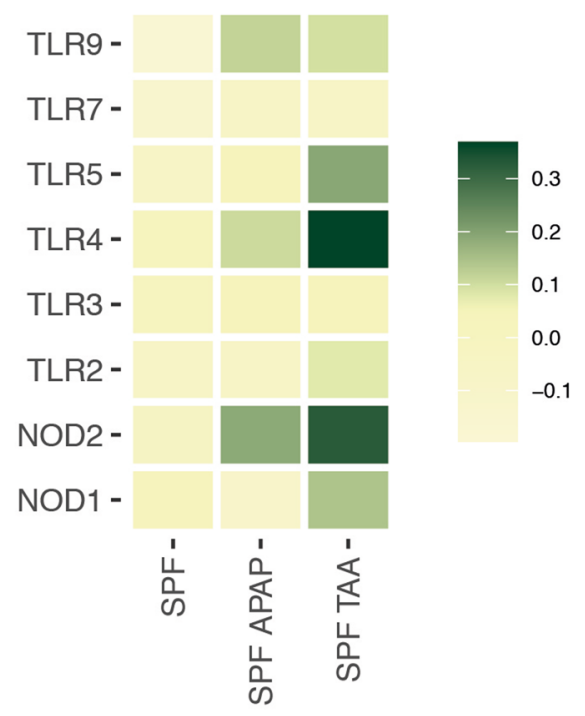

B
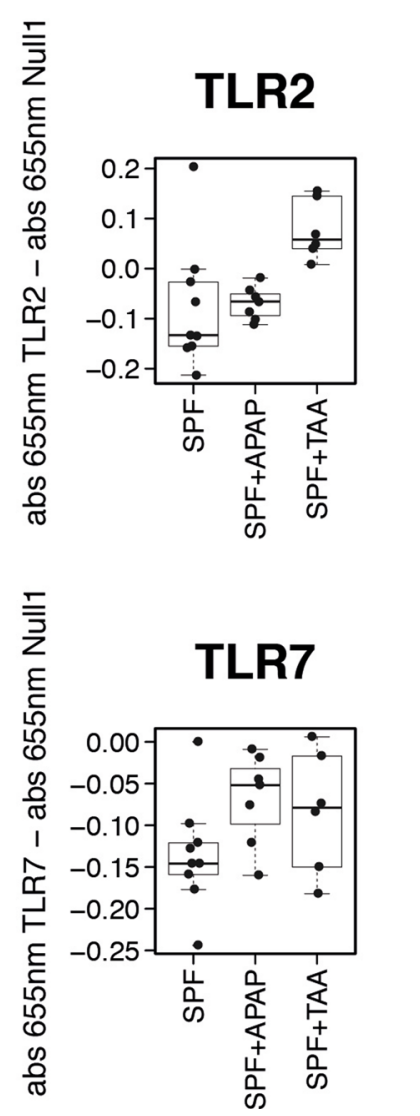
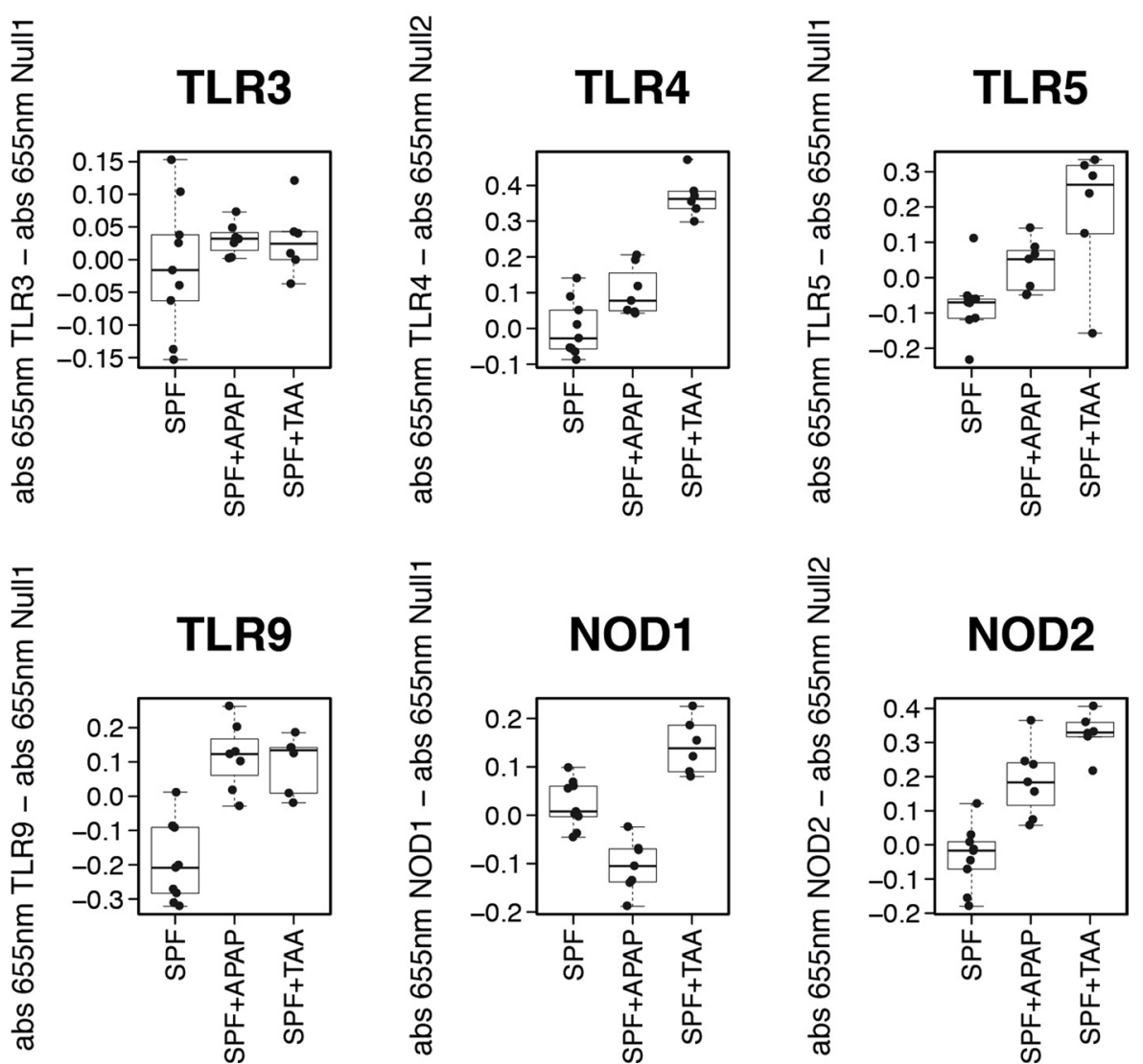

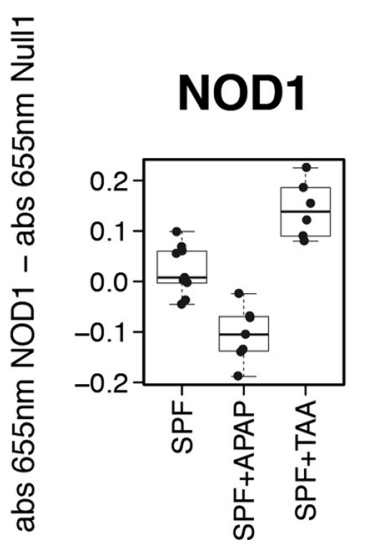

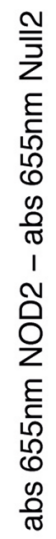

\section{NOD2}

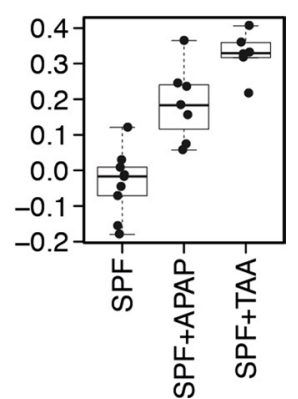

Extended Data Fig. 9 | TLR ligands in ALF. a, b, Heatmap and boxplots showing mean levels of $655 \mathrm{~nm}$ absorbance in HEK-Blue TLR and NLR reporter cell lines subtracted with absorbance of corresponding Null cell line after application of portal serum from germ free (GF), antibiotics treated (ABX) and specific pathogen free (SPF) mice treated with APAP and TAA. Boxplot defined as in Extended Data Fig. 1a. 


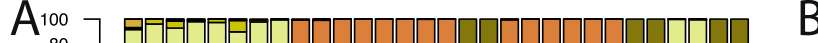
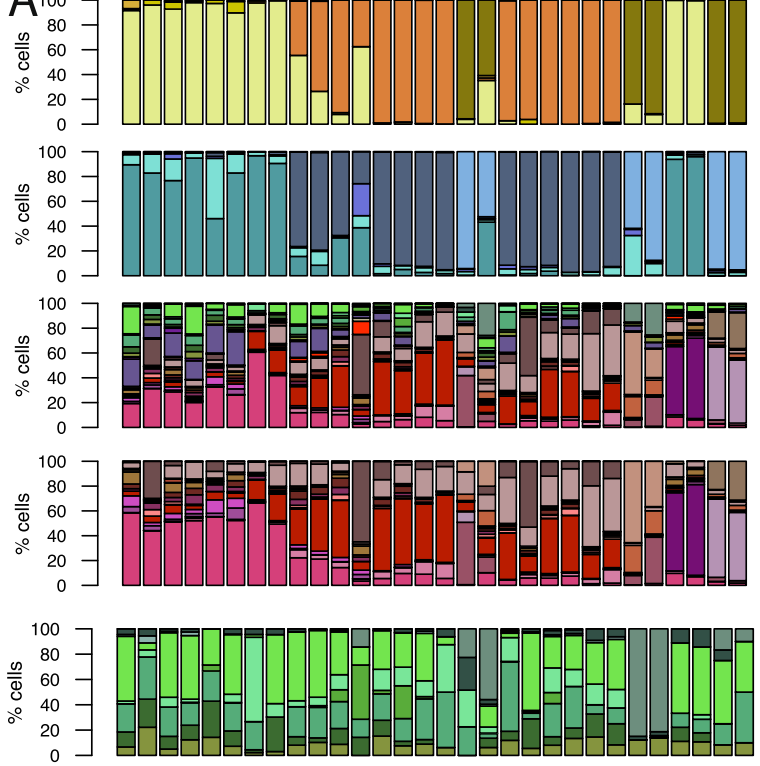

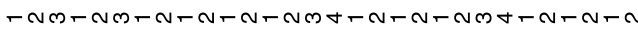

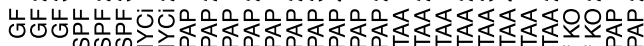

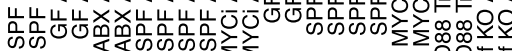
क员虫

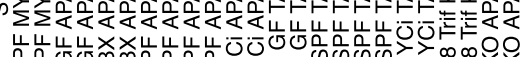

B
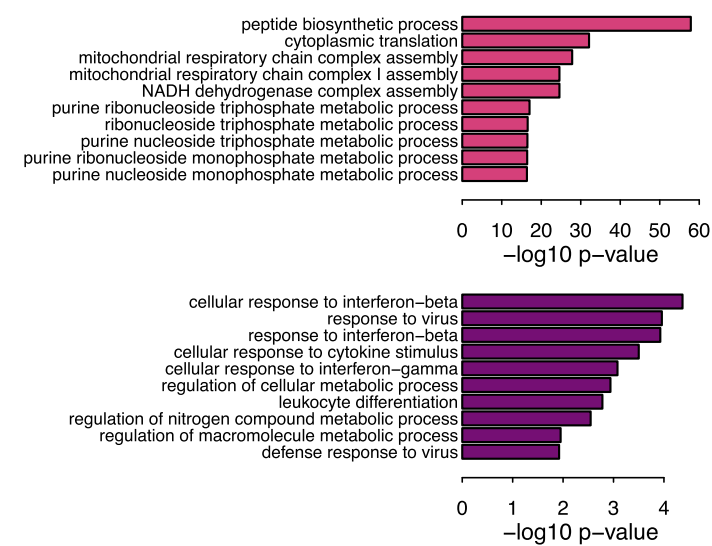

C
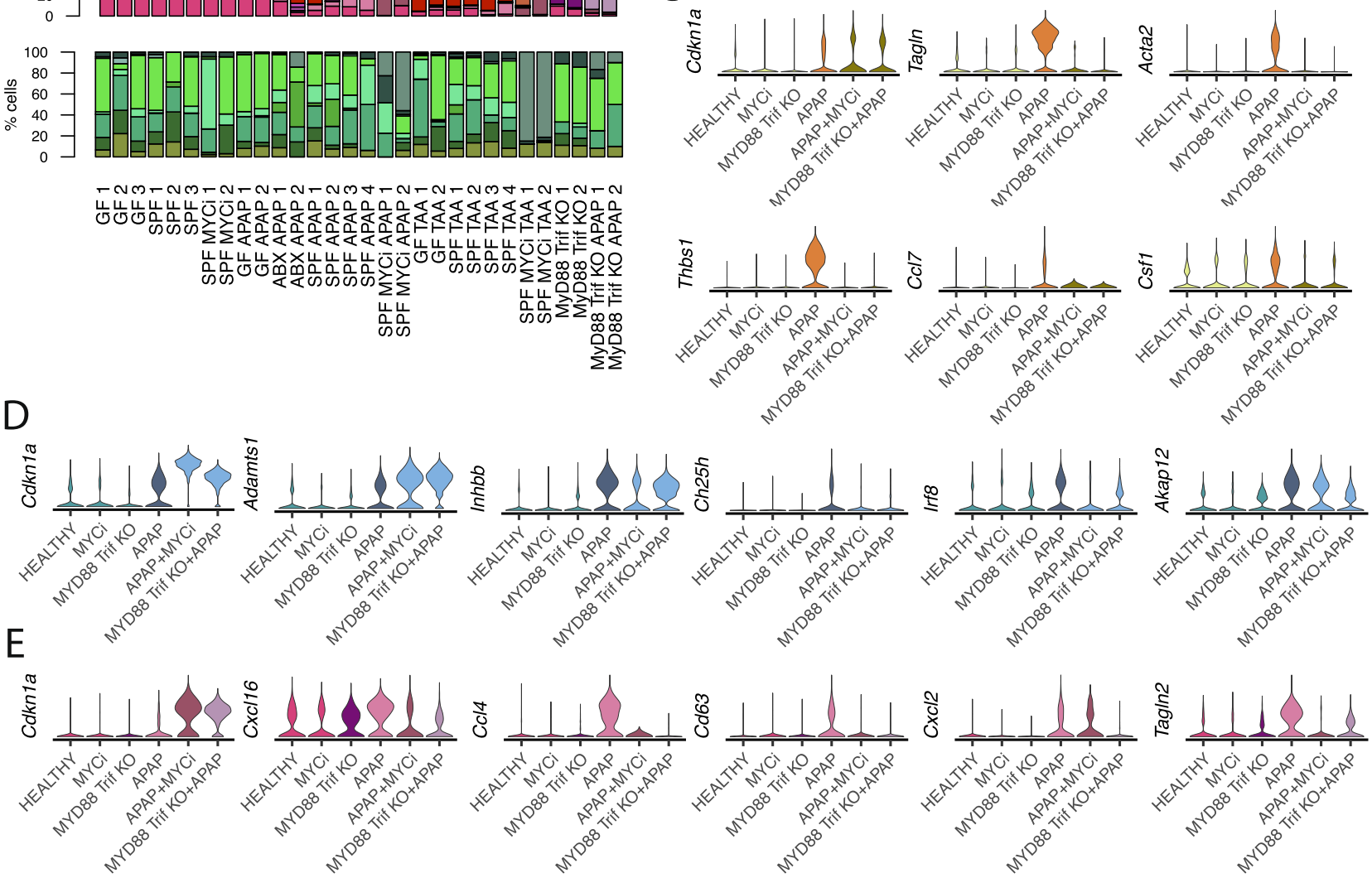

$\mathrm{F}$

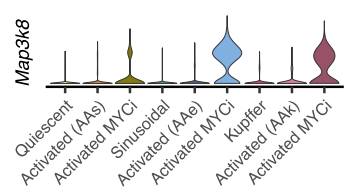

$\mathrm{G}$

\begin{tabular}{|c|c|c|l|}
\hline Sample & Age & Gender & \multicolumn{1}{|l|}{ Etiology } \\
\hline $19-05774$ & 72 & F & Liver donor \\
\hline $19-12173$ & 77 & M & Liver donor \\
\hline $18-20437$ & 32 & F & Liver donor \\
\hline $18-20858$ & 64 & M & Liver donor \\
\hline $18-10530$ & 63 & M & Liver donor \\
\hline $12-09634$ & 47 & M & Fulminant hepatic failure undetermined etiology \\
\hline $14-2115$ & 6 & M & Fulminant hepatic failure undetermined etiology \\
\hline $15-02328$ & 12 & F & Fulminant hepatic failure undetermined etiology \\
\hline $18-13822$ & 72 & M & Fulminant hepatic failure with autoimmune features \\
\hline $18-13906$ & 81 & F & Fulminant hepatic failure with autoimmune features \\
\hline $19-16184$ & 2 & M & Fulminant hepatic failure undetermined etiology \\
\hline $19-11747$ & 2 & F & Fulminant hepatic failure undetermined etiology \\
\hline
\end{tabular}

Extended Data Fig. 10 | See next page for caption. 
Extended Data Fig. 10 | Cellular response to APAP in MyD88 Trif KO mice. a, Relative frequencies of cells in healthy and mice with ALF in the presence and absence of MYCi as in Extended Data Fig. $4 f$ and additionally in GF, ABX and MyD88 Trif-dKO mice. b. Gene ontology term enrichment analysis of genes in Kupffer cells significantly more abundant in wild type (top) and significantly more abundant in Myd88-Trif dKO (bottom). GO analysis was performed with GProfiler using standard settings, p-values shown are corrected for multiple hypothesis testing using g:SCS algorithm c-e, Violin plots showing normalised and scaled expression of key genes in activated stellate cells (top), activated endothelial cells (middle) and activated Kupffer cells (bottom) in wild type mice, in presence of MYCi and in MyD88-Trif dKO mice. f, Violin plots showing normalised and scaled expression of TLP2 coding gene Map3k8 in activated resident populations in presence and absence of MYC inhibition. $\mathbf{g}$, Details of human liver samples including age in years, gender ( $\mathrm{F}=$ female, $\mathrm{M}=$ male) and disease status. 


\section{Reporting Summary}

Nature Research wishes to improve the reproducibility of the work that we publish. This form provides structure for consistency and transparency in reporting. For further information on Nature Research policies, see Authors \& Referees and the Editorial Policy Checklist.

\section{Statistics}

For all statistical analyses, confirm that the following items are present in the figure legend, table legend, main text, or Methods section.

$\mathrm{n} / \mathrm{a}$ Confirmed

$\bigotimes$ The exact sample size $(n)$ for each experimental group/condition, given as a discrete number and unit of measurement

Х A statement on whether measurements were taken from distinct samples or whether the same sample was measured repeatedly

The statistical test(s) used AND whether they are one- or two-sided

Only common tests should be described solely by name; describe more complex techniques in the Methods section.

$\bigotimes \square$ A description of all covariates tested

$\square$ A description of any assumptions or corrections, such as tests of normality and adjustment for multiple comparisons

A full description of the statistical parameters including central tendency (e.g. means) or other basic estimates (e.g. regression coefficient)

AND variation (e.g. standard deviation) or associated estimates of uncertainty (e.g. confidence intervals)

For null hypothesis testing, the test statistic (e.g. $F, t, r$ ) with confidence intervals, effect sizes, degrees of freedom and $P$ value noted

Give $P$ values as exact values whenever suitable.

Х $\square$ For Bayesian analysis, information on the choice of priors and Markov chain Monte Carlo settings

Х $\square$ For hierarchical and complex designs, identification of the appropriate level for tests and full reporting of outcomes

Х $\square$ Estimates of effect sizes (e.g. Cohen's d, Pearson's $r$ ), indicating how they were calculated

Our web collection on statistics for biologists contains articles on many of the points above.

\section{Software and code}

\section{Policy information about availability of computer code}

\section{Data collection No software was used.}

Data analysis bcl2fastq 2.17.1.14, CellRanger 2.1.1, Seurat 2.4.3, DESeq2 1.24.0, destiny 2.14.0, gProfileR 0.7.0, Image pro, FlowJo 8.8.6

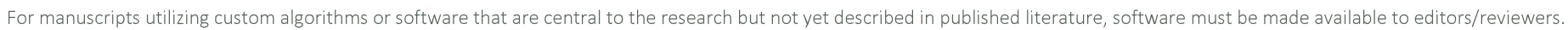
We strongly encourage code deposition in a community repository (e.g. GitHub). See the Nature Research guidelines for submitting code \& software for further information.

\section{Data}

Policy information about availability of data

All manuscripts must include a data availability statement. This statement should provide the following information, where applicable:

- Accession codes, unique identifiers, or web links for publicly available datasets

- A list of figures that have associated raw data

- A description of any restrictions on data availability

The scRNAseq data has been deposited at the ArrayExpress accession number E-MTAB-8263 and 16S sequencing data to ENA accession number ERP116956.

\section{Field-specific reporting}

Please select the one below that is the best fit for your research. If you are not sure, read the appropriate sections before making your selection.

$\bigotimes$ Life sciences $\quad \square$ Behavioural \& social sciences $\square$ Ecological, evolutionary \& environmental sciences

For a reference copy of the document with all sections, see nature.com/documents/nr-reporting-summary-flat.pdf 


\section{Life sciences study design}

All studies must disclose on these points even when the disclosure is negative.

Sample size This was an exploratory study of high throughput scRNAseq in acute liver failure. The number of samples per condition in single cell experiments was based on feasibility and great similarity between replicates.

Data exclusions No data was excluded

Replication We used 2 to 3 replicates of each single cell experiments to ensure reproducibility. ALT/AST and histology in APAP were done twice and all results were replicates (all data points are shown). 16S sequencing was done once, as it did not show any significant differences.

Randomization Allocation was not random. Covariates were controlled by processing the samples at the same place by the same people.

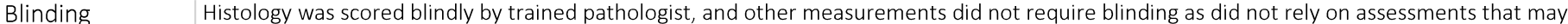
be subjective.

\section{Reporting for specific materials, systems and methods}

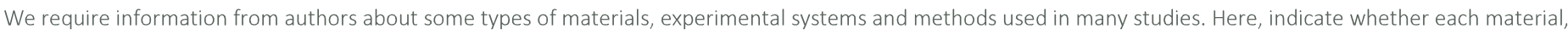

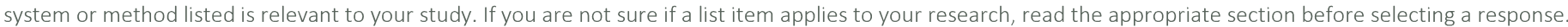

\begin{tabular}{l|l} 
Materials \& experimental sy \\
\hline$n / a$ & Involved in the study \\
$\square$ & $\bigotimes$ Antibodies \\
$\square$ & $\bigotimes$ Eukaryotic cell lines \\
$\square$ & $\bigotimes$ Animals and other organisms \\
$\square$ & $\square$ Human research participants \\
$\square$ & $\bigotimes$ Clinical data
\end{tabular}

Antibodies

Antibodies used

anti-CD45-PECy7 (Biolegend, 30F11, 1:1000, cat. 103114)

anti-F4/80-FitC (Serotec, Cl:A3-1, 1:1000, cat. G00018)

anti-Ly6C-APC (Biolegend, HK14, 1:1000, cat. 128016)

anti-cMYC monoclonal antibody (13-2500, 1:1000, Invitrogen)

anti-cMyc-Phospho-Ser62 (PA5-104729, 1:1000, Invitrogen)

Goat anti-mouse HRP (115-035-205, 1:5000, Jackson Labs)

Goat anti-rabbit HRP (111-035-003, 1:5000, Jackson labs)

Validation

These antibodies were verified for FACS by manufacturers and were used in many studies, as detailed in the manufacturers website

https://www.biolegend.com/en-us/products/pe-cy7-anti-mouse-cd45-antibody-1903

https://www.bio-rad-antibodies.com/monoclonal/mouse-f4-80-antibody-cl-a3-1-mca497.html?f=purified

https://www.biolegend.com/en-us/products/apc-anti-mouse-ly-6c-antibody-6047

https://www.thermofisher.com/antibody/product/c-Myc-Antibody-clone-9E10-Monoclonal/13-2500

https://www.thermofisher.com/antibody/product/Phospho-c-Myc-Ser62-Antibody-Polyclonal/PA5-104729

https://www.jacksonimmuno.com/catalog/products/115-035-205

https://www.jacksonimmuno.com/catalog/products/111-035-003

Eukaryotic cell lines

Policy information about cell lines

Cell line source(s)

Cell lines we purchased from Invivogen https://www.invivogen.com/hek-blue-tlr-cells

Authentication

Cell lines were not authenticated, as they were provided directly from Invivogen, positive controls were used to ensure no switching between lines 
Commonly misidentified lines (See ICLAC register)

\section{Animals and other organisms}

Policy information about studies involving animals; ARRIVE guidelines recommended for reporting animal research

Laboratory animals

Wild animals

Field-collected samples

Ethics oversight
C57BL6 wild type mice, 8 weeks old male mice

MyD88 and Trif double knockout mice were 8 weeks old, males in C57BL6 background

Note that full information on the approval of the study protocol must also be provided in the manuscript.

\section{Clinical data}

Policy information about clinical studies

All manuscripts should comply with the ICMJE guidelines for publication of clinical research and a completed CONSORT checklist must be included with all submissions.

Clinical trial registration

Study protocol

Data collection

Outcomes
The human data included in this paper is observational, focused on histology slides of de-idetnified individuals

All human studies were approved by the Weizmann Institute of Science Bioethics and Embryonic Stem Cell Research oversight committee, IRB approval number 699-1 and by the Rabin Medical Center Institutional Review Board, approval number: RMC-19-0816

\section{N/A}

N/A

\section{Flow Cytometry}

\section{Plots}

Confirm that:

$\bigotimes$ The axis labels state the marker and fluorochrome used (e.g. CD4-FITC).

\The axis scales are clearly visible. Include numbers along axes only for bottom left plot of group (a 'group' is an analysis of identical markers).

$\square$ All plots are contour plots with outliers or pseudocolor plots.

$\bigotimes$ A numerical value for number of cells or percentage (with statistics) is provided.

\section{Methodology}

Sample preparation
Measurement of monocyte infiltration

Livers of the mice were finely chopped with sterile scissors and then digested with $4 \mathrm{ml}$ of prewarmed $0.4 \mathrm{mg} / \mathrm{ml}$ protease and $0.1 \mathrm{U} / \mathrm{ml}$ collagenase D (EBS buffer: $8 \mathrm{~g} / \mathrm{l} \mathrm{NaCl}, 0.4 \mathrm{~g} / \mathrm{l} \mathrm{KCl}, 88 \mathrm{mg} / \mathrm{l} \mathrm{NaH2PO} \cdot \mathrm{H} 2 \mathrm{O}, 120 \mathrm{mg} / \mathrm{l} \mathrm{Na2HPO} 4 \cdot \mathrm{H} 2 \mathrm{O}, 2.38 \mathrm{~g} / \mathrm{l} \mathrm{HEPES}, 0.35 \mathrm{~g} / \mathrm{l}$ NaHCO3, $0.42 \mathrm{~g} / \mathrm{l} \mathrm{CaCl}$ ) for 30 minutes at $37 \mathrm{C}$, shaking. Then $10 \mathrm{ml}$ of cold PBS was added and the suspension was filtered through 100um mesh. To deplete hepatocytes samples were centrifuged at $30 \mathrm{~g}$ for 5 minutes and the supernatant was transferred to new tubes. Cells were collected by centrifugation at $580 \mathrm{~g}$. To lyse red blood cells, $1 \mathrm{ml}$ of Gibco ${ }^{\text {TM }}$ ACK Lysing Buffer was added and cells were incubated at room temperature for one minute. Subsequently, cold PBS was added and cells were collected by centrifugation at $580 \mathrm{~g}$.

Receptors on the cells were first blocked with TruStain FcX anti-mouse CD16/32, then the cells were washed with FACS buffer (PBS without calcium and magnesium, 1\% FCS), collected at 580g. and stained with CD45-PECy7 (Biolegend, 30F11, cat. 103114), F4/80-FitC (Serotec, Cl:A3-1, cat. G00018) and Ly6C-APC (Biolegend, HK14, cat. 128016) antibodies for 1 h on ice. Finally, the cells were washed with FACS buffer, collected at 580g, resuspended in FACS buffer.

Enrichment of stellate cells -

Liver cells were isolated using modified protocol by Mederacke and colleagues.16 Briefly, using a peristaltic pump we performed retrograde liver perfusion from inferior vena cava with three solutions: first EGTA solution $(8 \mathrm{~g} / \mathrm{l} \mathrm{NaCl}, 0.4 \mathrm{~g} / \mathrm{l} \mathrm{KCl}, 88 \mathrm{mg} / \mathrm{l}$ NaH2PO4.H2O, 12Omg/l Na2HPO4.H2O, 2.38g/l HEPES, 0.35g/l NaHCO3, 0.19g/l EGTA, 0.9g/l glucose)) for 2 minutes, second pronase solution $(0.4 \mathrm{mg} / \mathrm{ml}$ protease in EBS buffer: $8 \mathrm{~g} / \mathrm{l} \mathrm{NaCl}, 0.4 \mathrm{~g} / \mathrm{l} \mathrm{KCl}, 88 \mathrm{mg} / \mathrm{l} \mathrm{NaH2PO} \cdot \mathrm{H} 2 \mathrm{O}, 12 \mathrm{mg} / \mathrm{l} \mathrm{Na} 2 \mathrm{HPO} 4 \cdot \mathrm{H} 2 \mathrm{O}, 2.38 \mathrm{~g} / \mathrm{l}$ HEPES, $0.35 \mathrm{~g} / \mathrm{l} \mathrm{NaHCO}, 0.42 \mathrm{~g} / \mathrm{I} \mathrm{CaCl} 2)$ for 5 minutes and third with collagenase $D$ solution $(0.1 \mathrm{U} / \mathrm{ml}$ collagenase $D$ in EBS buffer $)$ for 7 minutes. Then liver was dissected, placed in cold EBS solution and shaken vigorously with forceps for the single cells to separate. The solution was filtered through 100um mesh and first hepatocytes were depleted by centrifugation at 30g for 5 


\section{Software}

FlowJo

Cell population abundance

Gating strategy
The aim of sorting was to enrich hepatic stellate cells, not to achieve high purity

Measurement of monocyte infiltration

FSC-A \& SSC-A (select all cells) -> SSC-W \& SSC-A (select singlets) -> PE-Cy7 \& Pacific Blue (select PE-Cy7 positive population i.e. CD45+ immune cells) $\rightarrow$ APC \& FitC (select double positive population)

Each of the population created clear separate cloud, on which we gated.

Enrichment of stellate cells

We gated on intrinsic fluorescence of retinol

FSC-A \& SSC-A (select all cells) -> SSC-W \& SSC-A (select singlets) -> Pacific Blue \& FitC (select Pacific blue positive cells)

\Tick this box to confirm that a figure exemplifying the gating strategy is provided in the Supplementary Information. 